\title{
WestVirginiaUniversity
}

THE RESEARCH REPOSITORY @ WVU

Graduate Theses, Dissertations, and Problem Reports

2007

\section{Image segmentation for biometric identification systems}

\author{
Eyad Haj Said \\ West Virginia University
}

Follow this and additional works at: https://researchrepository.wvu.edu/etd

\section{Recommended Citation}

Said, Eyad Haj, "Image segmentation for biometric identification systems" (2007). Graduate Theses, Dissertations, and Problem Reports. 4332.

https://researchrepository.wvu.edu/etd/4332

This Dissertation is protected by copyright and/or related rights. It has been brought to you by the The Research Repository @ WVU with permission from the rights-holder(s). You are free to use this Dissertation in any way that is permitted by the copyright and related rights legislation that applies to your use. For other uses you must obtain permission from the rights-holder(s) directly, unless additional rights are indicated by a Creative Commons license in the record and/ or on the work itself. This Dissertation has been accepted for inclusion in WVU Graduate Theses, Dissertations, and Problem Reports collection by an authorized administrator of The Research Repository @ WVU.

For more information, please contact researchrepository@mail.wvu.edu. 


\title{
Image Segmentation for Biometric Identification Systems
}

\author{
Eyad Haj Said \\ Dissertation submitted to the \\ College of Engineering and Mineral Resources \\ at West Virginia University \\ in partial fulfillment of the requirements \\ for the degree of \\ Doctor of Philosophy \\ in \\ Computer Engineering
}

Hany H. Ammar Ph.D, Committee Chairperson Arun Ross, Ph.D Xin Li, Ph.D

Sam Mukdadi, Ph.D Mohamed Abdel-Mottaleb, Ph.D

Lane Department of Computer Science and Electrical Engineering

Morgantown, West Virginia 2007

Keywords: Biometrics, Image Segmentation, Automated Segmentation Evaluation, ADIS, AEIS

Copyright 2007 Eyad Haj Said 


\title{
Abstract \\ Image Segmentation for Biometric Identification \\ Systems
}

\author{
Eyad Haj Said
}

Automating the identification process of individuals is receiving increased attention. Fully automated image segmentation from different types of biometric images is an essential step for designing automated identification systems. In this work, we address the problem of fully automated image segmentation in the context of dental and ear biometrics. We also address the problem of evaluating the quality of segmented image by designing an automated segmentation evaluator.

For image segmentation, we first apply a mathematical morphology operator to highlight the desired objects and suppress the others, and then threshold the resulting image to separate the desired objects from the background. We next analyze the connected components obtained from the thresholded image based on their geometric properties in order to isolate the desired objects. Results on dental radiograph images show that our approach performs very well compared to the other automated approaches. In addition, it has the lowest failure rate and highest optimality, and can deal not only with the bitewing views but also with the periapical views.

In ear images, our segmentation approach achieves more than $90 \%$ accuracy based on three different sets of 3750 facial images for 376 persons

We also present an approach for the automated evaluation of the quality of segmented images. Our approach is based on low computational-cost appearance-based features, and consists of two stages: off-line and on-line. In the off-line stage, we generate training sets by manually classifying the segmentation outcomes of proposed approach into several subclasses. Next, we create the Eigen-spaces corresponding to the different training sets. In the on-line stage, we project the outcome of segmentation onto the Eigen-spaces after view normalization, and use a Bayesian Classifier in order to determine whether the segmentation outcome is proper or improper segment. Experimental results for evaluating the segmentation outcomes of dental images and ear images indicate the benefits of the scheme. 


\section{Dedication}

I dedicate my thesis to my parents, wife, son, every member of my family, my mentors, and to all those who help me in this work. 


\section{ACKNOWLEDGMENTS}

The project of Automated Dental Identification System is supported in part by the U.S. National Science Foundation under Award number EIA-0131079 to West Virginia University; the research is also supported under Award number 2001-RC-CX-K013 from the Office of Justice Programs, National Institute of Justice, and the U.S. Department of Justice. Points of view in this document are those of the authors and do not necessarily represent position of the U.S. Department of Justice.

Ear project is funded by the Federal Bureau of Investigation's Forensic Audio Video Image Analysis Unit (FAVIAU) . The administration and contracting is being done through the federal government's Technical Support Working Group (TSWG). Dr. Nicole Spaun and Richard Vorder Bruegge of the FAVIAU are the FBI's technical advisors for this project.

The author wish to thank Dr. Anil K. Jain and Hong Chen of Michigan State University as well as Dr. Mohamed Abdel-Mottaleb, Omaima Nomair, and Jindan Zhou of University of Miami for providing the authors with implementations of their referenced teeth and ear segmentation algorithms 


\section{TABLE OF CONTENTS}

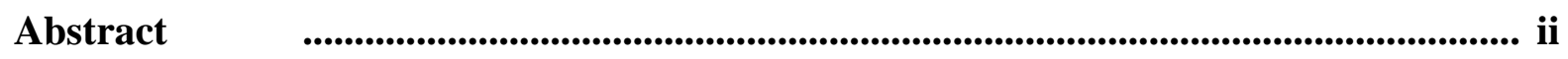

Dedication $\quad$....................................................................................................................................ii

Acknowledgements ...................................................................................................................... iv

Table of contents ..................................................................................................................................... V

List of Figures $\quad$.......................................................................................................................................... vii

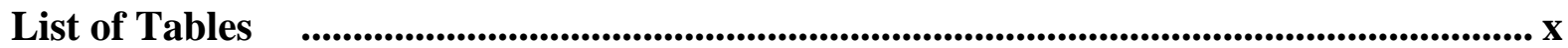

Chapter 1: Introduction ....................................................................................................... 1

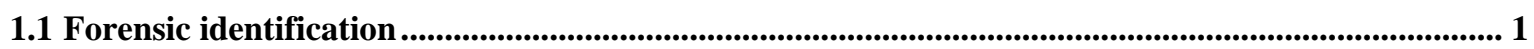

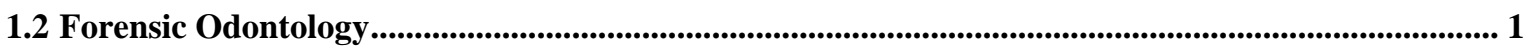

1.3 Automated Dental identification System (ADIS) ........................................................................................ 3

1.4 Automated Ears Identification System (AEIS) ................................................................................................ 6

Chapter 2: Problem Statement and Research Objective ............................................................ 9

2.1 Dental Image Segmentation ............................................................................................................................ 9

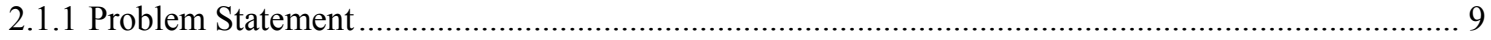

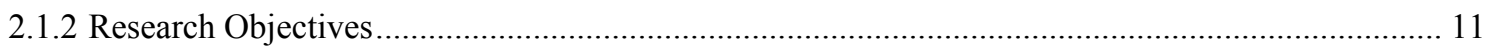

2.2 Dental Image Segmentation Automated Evaluation.................................................................... 11

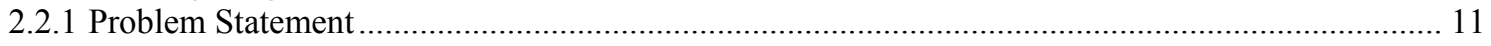

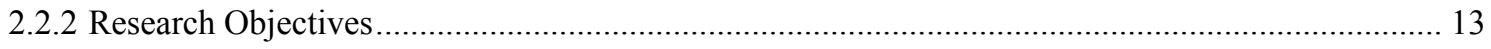

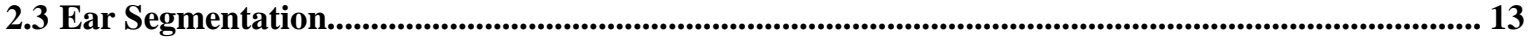

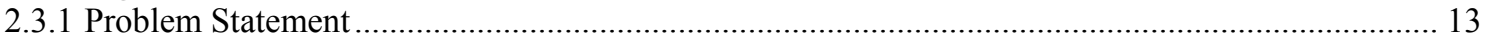

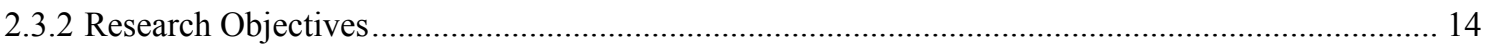

Chapter 3: Literature Survey ........................................................................................................... 15

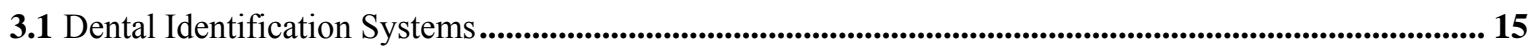

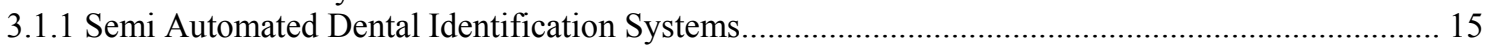

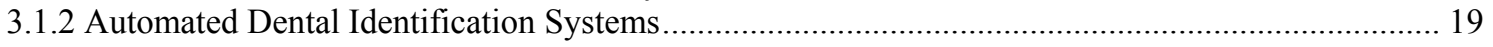

3.2 Ear Identification systems................................................................................................................................... 20

3.3 Image Segmentation ............................................................................................................................................... 22

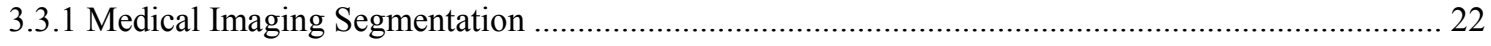

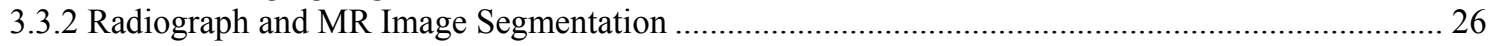

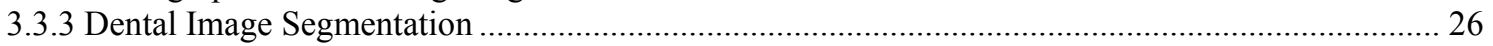

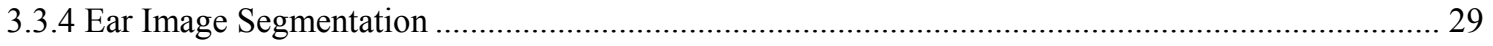

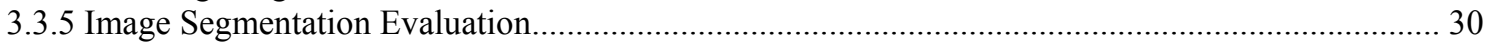

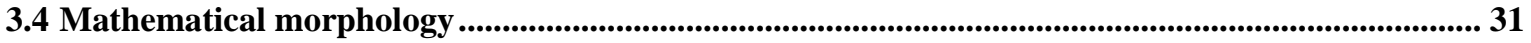

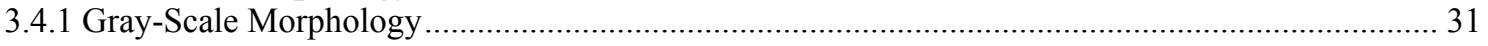

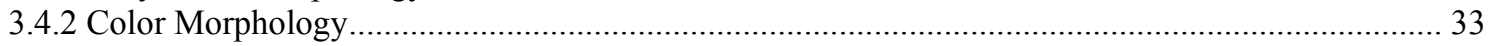

3.4.3 Medical Applications of Mathematical Morphology in Image segmentation ............................... 34 


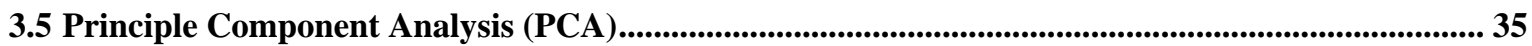

3.4.1 Principle Component Analysis Applications ………….............................................................. 38

Chapter 4: Contribution......................................................................................... 41

Chapter 5: Approach for Segmentation Algorithm................................................. 43

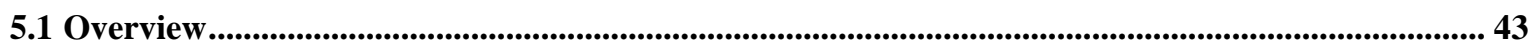

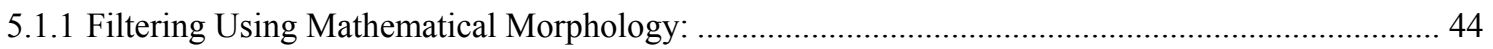

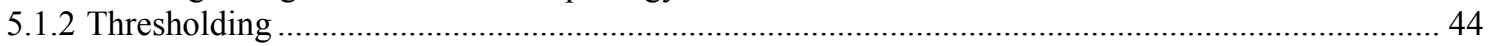

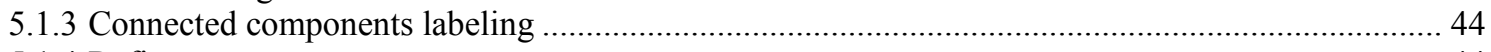

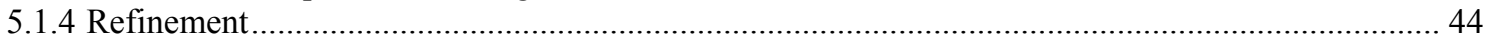

5.2 Teeth Segmentation from Dental x-rays Images............................................................................. 45

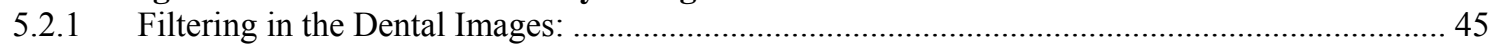

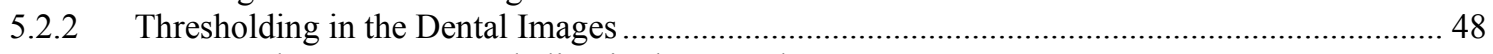

5.2.3 Connected Components Labeling in the Dental Images ............................................................ 49

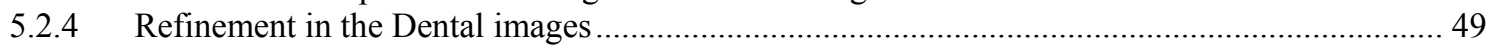

5.3 Performance Assessment in the Dental Image Segmentation .................................................................... 52

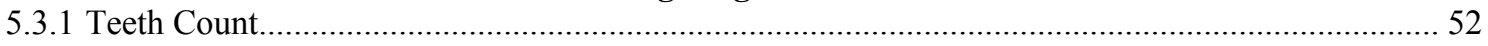

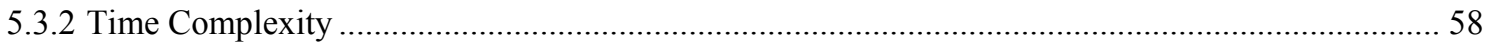

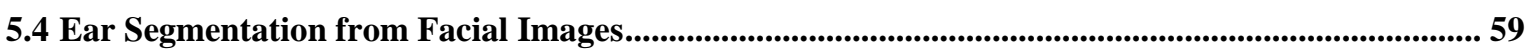

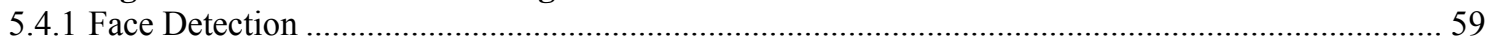

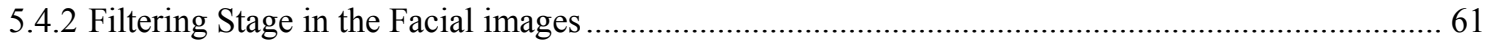

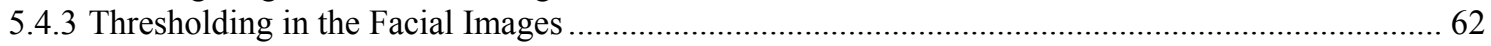

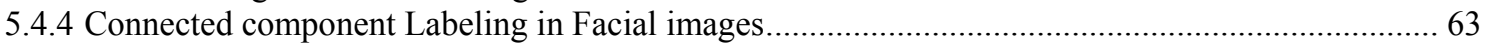

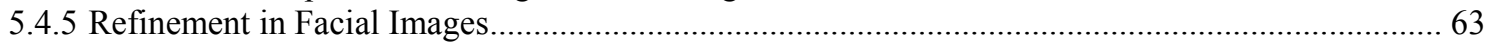

5.5 Performance Assessment of Ear Segmentation........................................................................................... 66

5.6 Advanced Approach for Dental Image Segmentation .................................................................................. 68

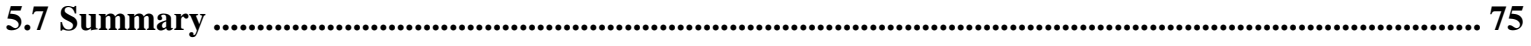

Chapter 6: Approach for Automated Segmentation Evaluator ASE ....................... 78

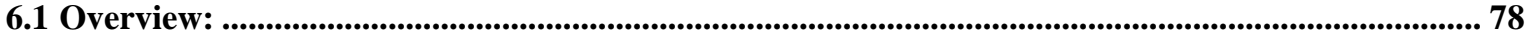

6.2 Manual Classification of the segmentation outcomes:.................................................................................... 79

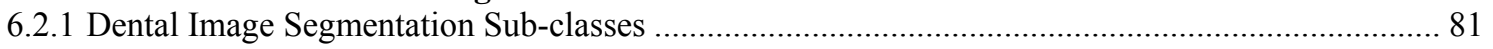

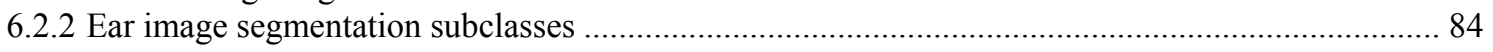

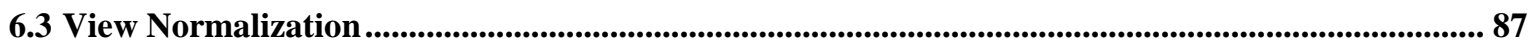

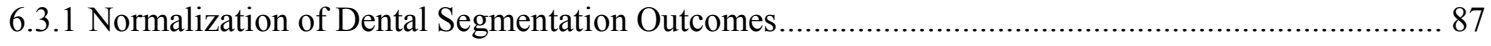

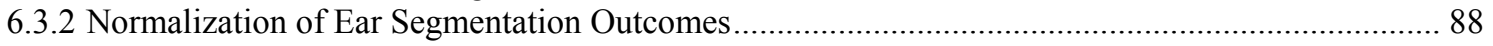

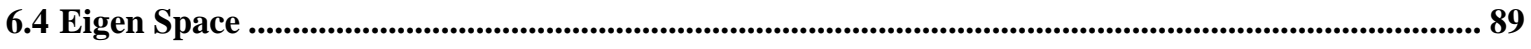

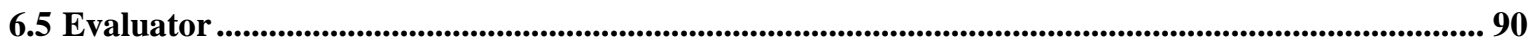

6.6 Experimental Results for Dental Image Segmentation Evaluation .................................................... 93

6.7 Experimental Results for Ear Image Segmentation Evaluation ............................................................. 102

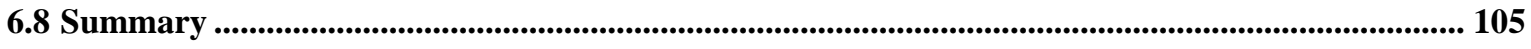

Chapter 7: Conclusion.................................................................................................................. 107

Bibliography $\quad$.................................................................................................................................. 109 


\section{LIST OF FIGURES}

Figure 1: Different types of dental x-rays views. .............................................................. 3

Figure 2: A block diagram illustrating the architecture of ADIS. ....................................... 5

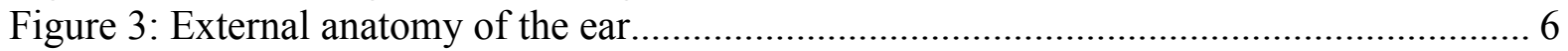

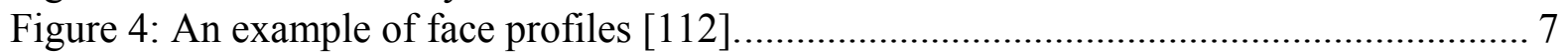

Figure 5: Block diagram illustrating the architecture of AEIS. ..................................... 8

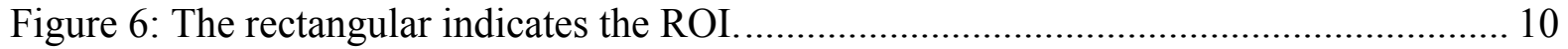

Figure 7: Examples of difficulties that could face dental image segmentation.................... 10

Figure 8: Examples of over segmentation results, (a) teeth interfering and filings, (b) fillings,

(c) teeth interfering, (d) teeth interfering between the upper jaw and lower jaw. ... 12

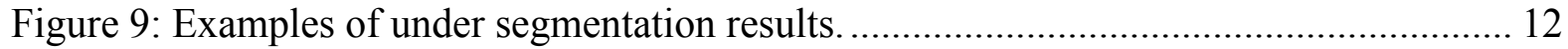

Figure 10: Examples of segmentation results that related to bones or background............... 12

Figure 11: (a) Integral projection on Y axis, (b) Integral projection of pixels of the upper jaw along the lines perpendicular to the curve of the valley, (c) the segmentation result.

Figure 12: Two different results for same image. (a) Completely segmented image.(b) Failed

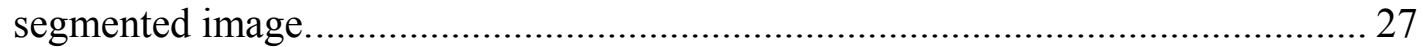

Figure 13: (a) Original Image, (b) the result of MM filtering, (c) the segmentation result.... 28

Figure 14: Tabular presentation of segmentation results (adopted from [84]).................... 30

Figure 15: Main stages of proposed segmentation algorithm.......................................... 43

Figure 16: Grayscale line profiles of the input image, the upper horizontal line profile illustrates the bones between the teeth, the lower horizontal line profile shows the gap between the teeth, while the vertical line profile illustrates the gap valley..... 46

Figure 17: Grayscale line profiles of the closing top hat transformed image that are corresponded to the same line profiles in Figure 16 ........................................ 47

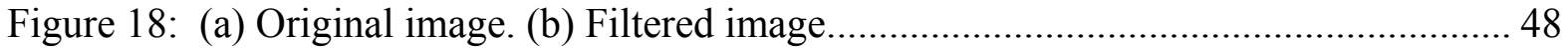

Figure 19: Cumulative histogram of the filtered image.................................................. 49

Figure 20: (a)-(c) Thresholded Images. (e)-(g) Result of connected component labeling for

(a)-(c). (g)-(i) Proper ROIs generated from (e)-(g)........................................... 50

Figure 21: (a) Original image. (b)-(i) Results of segmentation. ......................................... 51

Figure 22: (a) Original image, (b) Filtered image, (c) Thresholded Images ,(d) Result of connected component labeling, (e)-(h) Proper ROIs. ....................................... 51

Figure 23: Examples of fully successful images and their segmentation outcomes.............. 52

Figure 24: Examples of partially successful images and their segmentation outcomes ........ 52

Figure 25: Examples of completely failed images where no tooth can be detected............. 52

Figure 26: Testing results for of our teeth segmentation algorithm with bitewing views..... 53

Figure 27: Testing results for of our teeth segmentation algorithm with contrast stretched bitewing views.

Figure 28: Testing results for of our teeth segmentation algorithm with periapical views. ... 54

Figure 29: Testing results for of our teeth segmentation algorithm with contrast stretched

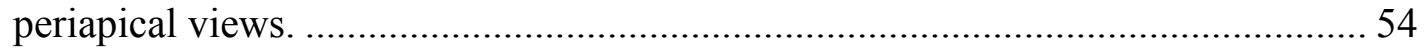

Figure 30: Testing results of Jain and Chen segmentation algorithm [8]. ......................... 55

Figure 31: Testing results of Nomair and Abdel-Mottaleb segmentation algorithm [9]. ....... 55

Figure 32: Testing results of Zhou and Abdel-Mottaleb segmentation algorithm [10]........ 55

Figure 33: Performance comparison between bitewing segmentation algorithms............... 56 
Figure 34: Performance comparison between proposed algorithm and proposed algorithm

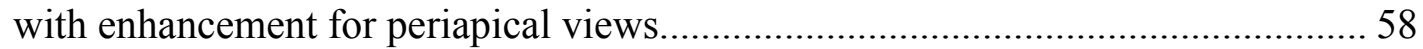

Figure 35: Time complexity distribution of 40 images for variants algorithms .................. 60

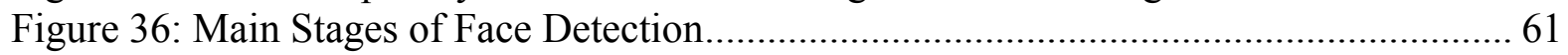

Figure 37: An Example shows the stages of face detection............................................ 61

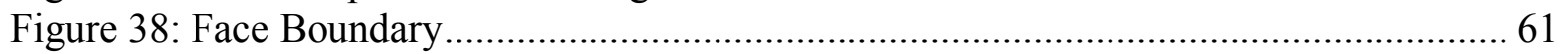

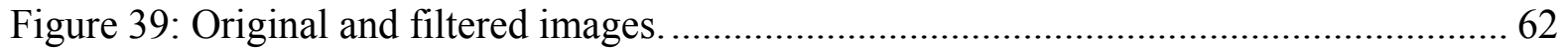

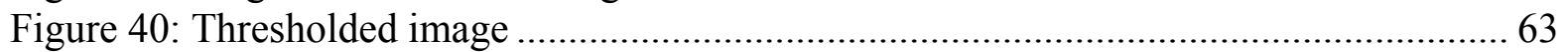

Figure 41: Result of connected component labeling of the thresholded image .....................63

Figure 42: Detected ear after refinement stage. ......................................................... 64

Figure 43: Examples of completely successful images and their segmentation outcomes

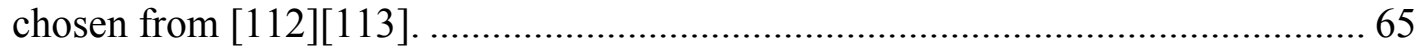

Figure 44: Examples of completely failed images and partially successful succeeded images and their segmentation outcomes chosen from [112][113] ................................6 65

Figure 45: Examples of completely successful images and their segmentation outcomes.... 66

Figure 46: Examples of completely failed and partially succeeded images and their

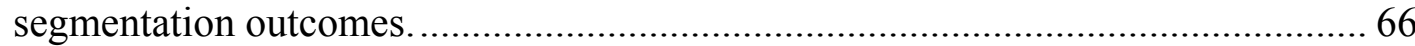

Figure 47: (a) Original image, (b) blurred image, (c) image resulted from subtracting the blurred image form its original .................................................................. 69

Figure 48: (a) Thresholded image, (b) thresholded image after filtering the small regions, (c)

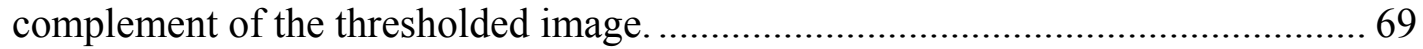

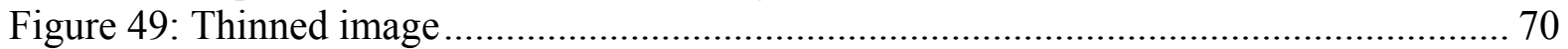

Figure 50: Examples of completely succeeded images. ............................................... 70

Figure 51: Examples of partially succeeded images..................................................... 70

Figure 52: Examples of completely failed images......................................................... 71

Figure 53: Testing results of our advanced teeth segmentation algorithm with bitewing

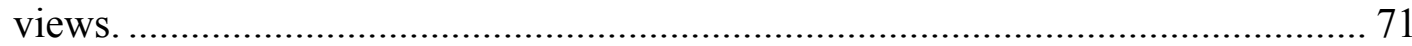

Figure 54: Testing results of our advanced teeth segmentation algorithm with periapical

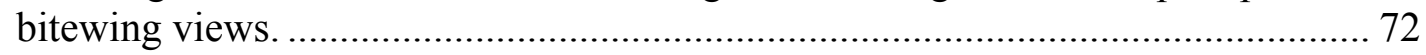

Figure 55: Performance comparison between bitewing segmentation algorithms............... 73

Figure 56: Performance comparison between segmentation algorithm proposed in section 5.2 and the one presented in section 5.6 for periapical views................................... 73

Figure 57: Testing results of our advanced teeth segmentation algorithm with 800 bitewing

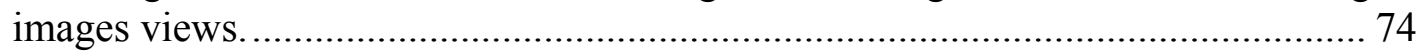

Figure 58: Testing results of our advanced teeth segmentation algorithm with 333 upper

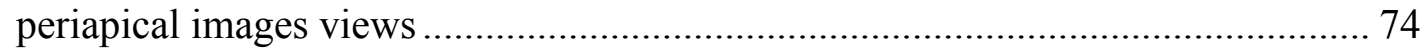

Figure 59: Testing results of our advanced teeth segmentation algorithm with 321 lower

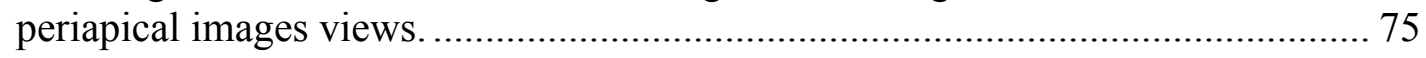

Figure 60: Automated Segmentation Evaluator ASE ................................................. 78

Figure 61: (a) off-line stage of ASE. (b) on-line stage of ASE. ...................................... 80

Figure 62: Samples of upper jaw teeth in the Bitewing images ...................................... 82

Figure 63: Samples of lower jaw teeth in the Bitewing images ...................................... 82

Figure 64: Samples of upper jaw oversegmentation outcomes in the Bitewing images ....... 82

Figure 65: Samples of lower jaw oversegmentation outcomes in the Bitewing images ........ 82

Figure 66: Samples of upper jaw undersegmentation outcomes in the Bitewing images ...... 83

Figure 67: Sample of lower jaw undersegmentation outcomes in the Bitewing images ........ 83 
Figure 68: Samples of non-teeth/background outcomes in the Bitewing images.................. 83

Figure 69: Samples of teeth in the upper jaw periapical images ...................................... 83

Figure 70: Samples of upper jaw oversegmentation outcomes in the upper jaw periapical dental images.

Figure 71: Samples of upper jaw undersegmentation outcomes in the upper jaw periapical

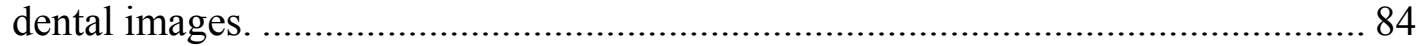

Figure 72: Samples of teeth in the lower jaw periapical images ..................................... 84

Figure 73: Samples of upper jaw oversegmentation outcomes in the lower jaw periapical dental images.

Figure 74: Samples of lower jaw undersegmentation outcomes in the lower jaw periapical

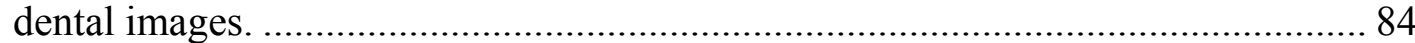

Figure 75: Samples of background outcomes in the periapical dental images.................... 84 Figure 76: Samples of proper ear segmentation outcomes in a angle view between [-10, 40].

Figure 77: Samples of proper ear segmentation outcomes in a angle view between [40, 80].85

Figure 78: Samples of partially succeeded ear segmentation outcomes in a angle view between

Figure 79: Samples of partially succeeded ear segmentation outcomes in a angle view

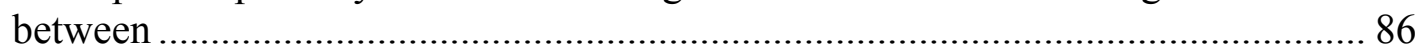

Figure 80: Samples of improper ear segmentation outcomes.......................................... 86

Figure 81: View normalization for different dental image segmentation sub-classes, Tooth, Oversegmentation, undersegmentation, and Background. (a) original segment, (b) edge image, (c) rotated edge image corresponding to strongest vertical edges, (d) normalized segment image. .................................................................... 88

Figure 82: View normalization of ear image segmentation outcome............................... 89

Figure 83: Variation in ASE performance of the Eigen-space method using different number of principal components for bitewing image segmentation outcomes

Figure 84: Variation in ASE performance of the Eigen-space method using different number of principal components for upper periapical image segmentation outcomes........ 97

Figure 85: Variation in ASE performance of the Eigen-space method using different number of principal components for lower periapical image segmentation outcomes........ 97

Figure 86: Examples of correctly evaluated segments. .................................................. 99

Figure 87: Examples of incorrectly evaluated segments ............................................... 99

Figure 88: Confusion matrixes for various views of dental images. .............................. 100

Figure 89: Examples of correctly evaluated segments. ................................................. 103

Figure 90: Examples of incorrectly evaluated segments. ................................................ 104

Figure 91: ROC Curve of matcher performance using Ideal segmentation, segmentation without ASE, and segmentation with presence of ASE .................................... 105 


\section{LIST OF TABLES}

Table 1: Summary of comparison between three teeth segmentation algorithms [8][9][10]. 28

Table 2: Rules used in refinement stage to determine the proper ROIs.

Table 3: Comparison between the failure rates of the variant teeth segmentation algorithms.

Table 4: Comparison between the time complexities of the four teeth segmentation algorithms.

Table 5: Comparison results between our approach and the approach presented in [11] for the first testing set [113]

Table 6: Comparison results between our approach and the approach presented in [11] for the second testing set [112]

Table 7: Comparison results between our approach and the approach presented in [11] for the third testing set [114].

Table 8: Percentage of optimality, failure, and sub-optimality of our advanced teeth segmentation for various dental images views.

Table 9: Classification of teeth segmentation outcomes for various views of dental x-rays radiographs, bitewing, upper periapical, and lower periapical

Table 10: The number of each subclass resulted from dental image segmentation for bitewing dental images.

Table 11: The number of each subclass resulted from dental image segmentation for upper jaw periapical images.

Table 12: The number of each subclass resulted from dental image segmentation for lower jaw periapical images.

Table 13: The number of each subclass resulted from dental image segmentation for bitewing dental images after evaluation.

Table 14: The number of each subclass resulted from dental image segmentation for upper jaw periapical images after evaluation................................................................ 98

Table 15: The number of each subclass resulted from dental image segmentation for lower jaw periapical images after evaluation.

Table 16: Result of Matching PM records against AM records for 1 2Subject using ADIS with/without ASE.

Table 17: Error rates vs. of number of principle components for ear and non ear segments 103 


\section{Chapter 1: Introduction}

\subsection{Forensic identification}

Biometric systems play an important role in identifying individuals based on some physical or behavioral characteristics [1], like fingerprints, retina, iris, face, hand geometry, voice, and signature.

Forensic identification is typically defined as the use of science or technology in identifying human beings in a court of law. It has broad applications in criminal investigations, court evidences and security applications. With the evolution of information technology and the large volume of cases that need to be investigated by forensic specialists, automation of forensic identification has become inevitable.

Forensic identification may take place prior to death and this is referred to as Antemortem (AM) identification. Identification may also be carried out after death and this is called Postmortem (PM) identification. While most physical and behavioral characteristics such as fingerprints, iris, face, etc..., are not suitable for Postmortem (PM) identification, especially under the severe circumstances typically encountered in natural and artificial disasters (e.g. Tsunami, aviation disasters), dental features are one of the few biometric identifiers that can be used for PM identification. Dental features are manifested in the root and crown morphology, dimensions of the teeth, spacing between teeth and sinus patterns, as well as characteristics of dental work and restorations[2] [3].

\subsection{Forensic Odontology}

Forensic odontology (also called Forensic Dentistry) is a branch of forensics concerned with performing variety of tasks including identifying human individuals based on their dental features and bite mark analysis associated with human abuse. Traditionally, forensic odontologists have relied on the morphology of dental restorations (fillings, crowns, etc.) to identify victims. However, the present generation undergoes less dental decay than their predecessors. Consequently, it is becoming important to establish identification based on other dental features like root and crown morphology, rotations, spacing between teeth and sinus patterns, etc [2][3].

There are several forms of dental records that are being used by forensic odontologists, the most common of which are dental radiographs (x-rays), oral photographs, dental models, and 
CAT scans. In most postmortem identification cases, dental radiographic records (twodimensional x-ray images) are the prime tools that a forensic odontologist would employ. Dental x-ray images are classified according to the view they are taken from and their coverage. The most commonly used dental $\mathrm{x}$-ray image types are: bite-wing $\mathrm{x}$-ray, periapical $\mathrm{x}$-rays, and panoramic x-rays [5] as shown in Figure 1:

1. Bite-wing $x$-rays are acquired to view the back teeth, and they are the typical $x$-rays a dentist will take because of their effectiveness in discovering tooth decay. In a bitewing $\mathrm{x}$-ray, only the crowns and part of the roots are seen for 2 or 3 adjacent teeth in both the upper and lower jaws.

2. Periapical x-rays are taken to facilitate the most effective examination of the entire tooth area including the tip of the root and the surrounding tissues. They are appropriate to confirm a tooth is impacted or if there are problems at the end of the root, such as root fracture and deep decay. This type of $\mathrm{x}$-rays provides a complete side view and typically a complete set consists of 14-24 films with each tooth appearing in two different films from two different angles.

3. Panoramic $\mathrm{x}$-rays are taken to view both upper and lower jaws. They are usually taken on an initial visit to a dentist, as they are effective in capturing the overall dental status of a patient, showing for example, impacted teeth, eruption of permanent teeth, jaw fractures, tumors, and cysts. However, they do not exhibit the fine details as found in bite-wings and periapical x-rays. Often, bite-wing and periapical films are combined to form the so called "Full series", in which all teeth and surrounding tissues are shown, with very high definition details of the individual teeth. A forensic odontologist might have to compare two different types of dental radiographs to find similar dental features.

Figure 1 shows a single dental $\mathrm{x}$-rays record that contains the three main types of dental images: bitewing, periapical and panoramic. 


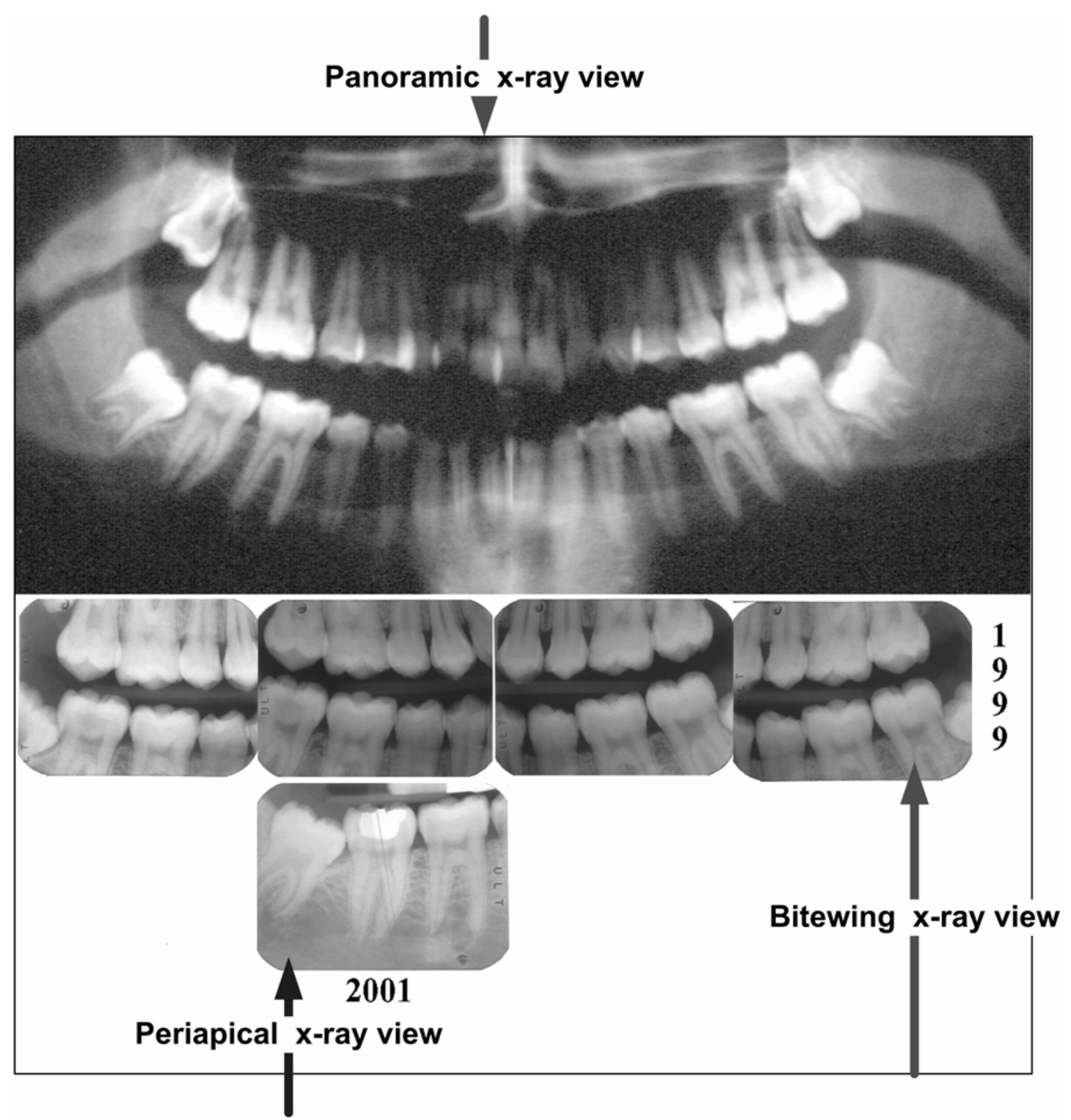

Figure 1: Different types of dental x-rays views.

\subsection{Automated Dental identification System (ADIS)}

Based on the information provided by experts from the Criminal Justice Information Services Division (CJIS) of the FBI, there are over 100,000 unsolved Missing Persons cases in the National Crime Information Center at any given point in time, 60 percent of which have remained in the computer system for 90 days or longer. In 1997, The Criminal Justice Information Services Division (CJIS) of the FBI created a dental task force (DTF) whose goal is to improve the utilization and effectiveness of the National Crime Information Center's (NCIC) Missing and Unidentified Persons 
(MUP) files. The DTF recommended the creation of a Digital Image Repository (DIR) and an Automated Dental Identification System (ADIS) [6] with goals and objectives similar to the Automated Fingerprint Identification System (AFIS) [7] but using dental characteristics instead of fingerprints. PM dental identification is mainly achieved by comparing a subject dental record to a database of dental records. Dental radiographs are the most common forms of dental records used in PM identification.

At a high level of abstraction, we view ADIS as a collection of the following megacomponents (as depicted in Figure 2):

1. The Record Preprocessing component handles cropping or segmentation of dental records (which contain different views) into dental films; enhancement of films; classification of films into bitewing, periapical, or panoramic views; segmentation of teeth from films; and annotating teeth with labels corresponding to their location in a dental atlas.

2. The Potential Match Search component manages the archiving and retrieval of dental records based on high-level dental features (e.g. number of teeth and their shape properties) and produces a candidate list.

3. The Image Comparison component performs low-level tooth-to-tooth comparison between the subject teeth -after alignment- and the corresponding teeth of each candidate, thus producing a short match list.

This framework broadly defines the collaborative research tasks between research teams from West Virginia University, Michigan State University, and University of Miami that are jointly developing a research prototype of ADIS [6]. However, ADIS is the first fully automated system presented for identification purposes using dental X-rays images. 


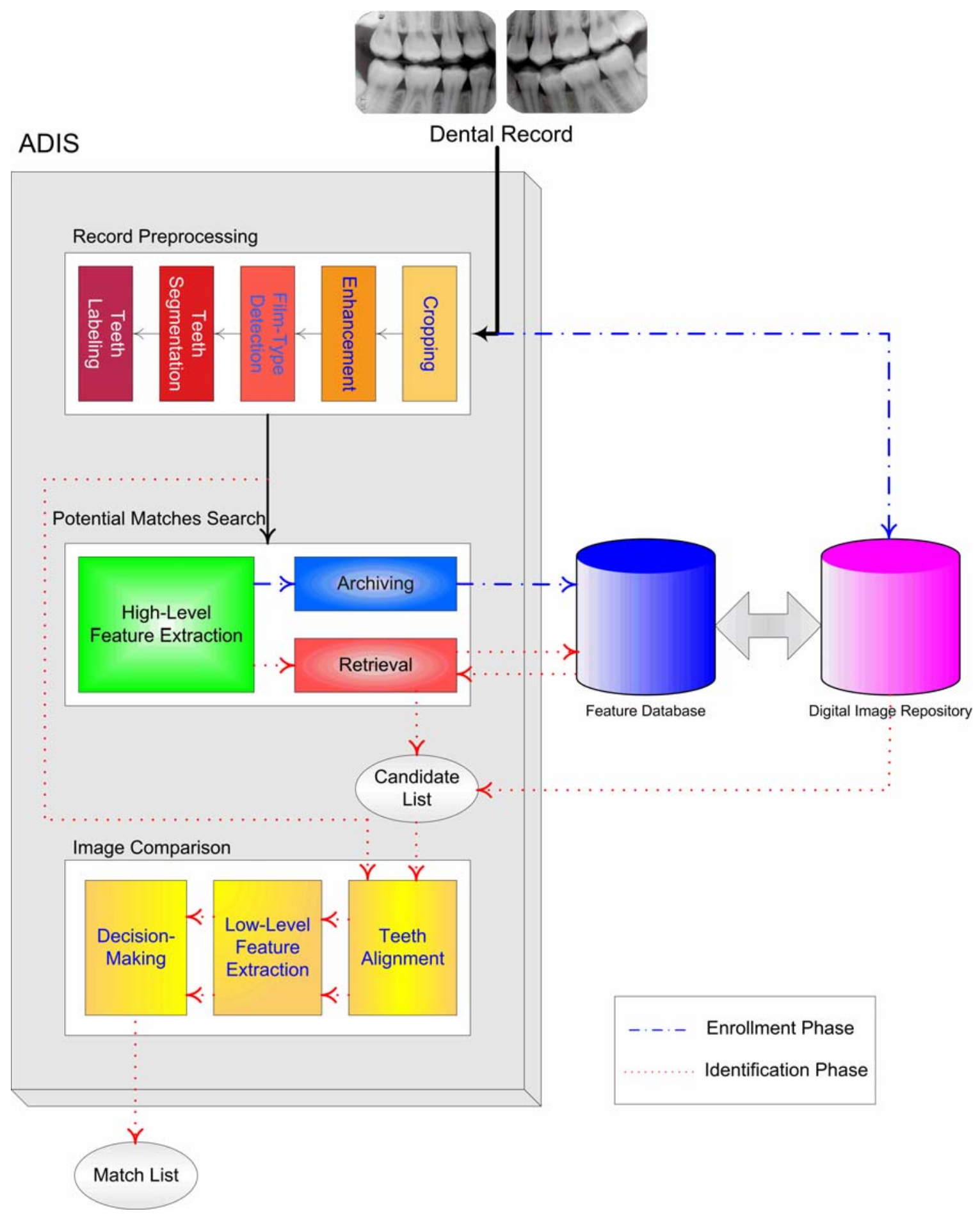

Figure 2: A block diagram illustrating the architecture of ADIS. 


\subsection{Automated Ears Identification System (AEIS)}

Several studies have explored the possibility of comparing ears based on their features and structures [4]. The studies show that the ear structure is not only unique but also stable and permanent [4]. Figure 3 shows the external anatomy of the ear and its nine components Helix Rim (1), Lobule (2), Antihelix (3), Concho (4), Tragus (5), Antitragus (6), Crus of helix (7), Triangular fossa (8), and Incisure intertragcia (9).

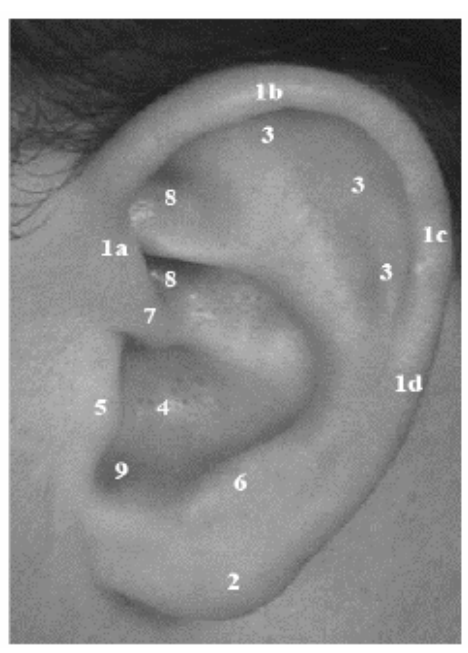

Figure 3: External anatomy of the ear.

Our goal is to design and develop an Automated Ear Identification System (AEIS) based on the 3-D and 2-D features of the ears. The input for AEIS is a video camera sequence or multiple images containing the subject face. Samples of video frames and images are shown in Figure 4 with different views. At a high level of abstraction, we view AEIS as a collection of the following components (as depicted in Figure 5):

1. The Preprocessing stage handles extracting image frames from the video sequence, enhancement of images to reduce the blurring effect thereby increasing the efficiency of the later steps, segmentation of ears, generating a high resolution image of ear with more details obtained from sequential frames, and extracting 3-D and 2-D features of the ear from different ear views.

2. Archiving and retrieval of the video frames and the corresponding ear, and producing a candidate list to facilitate the identification process. 
3. The Comparison stage where a ear-to-ear comparison is performed between the subject's ear and the corresponding ear of each candidate, thus producing a short match list and match score for each candidate.
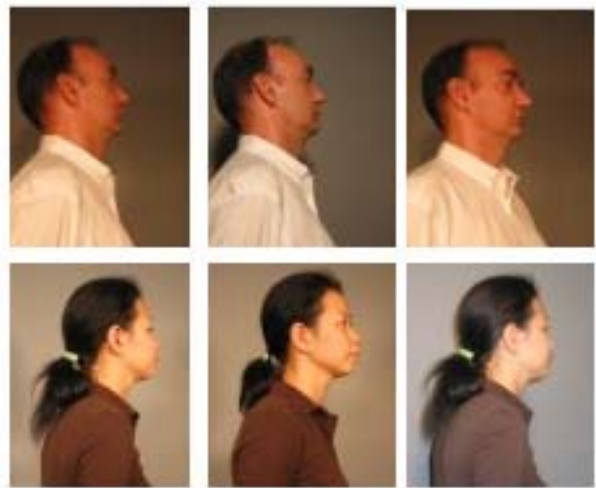

Figure 4: An example of face profiles [112]. 


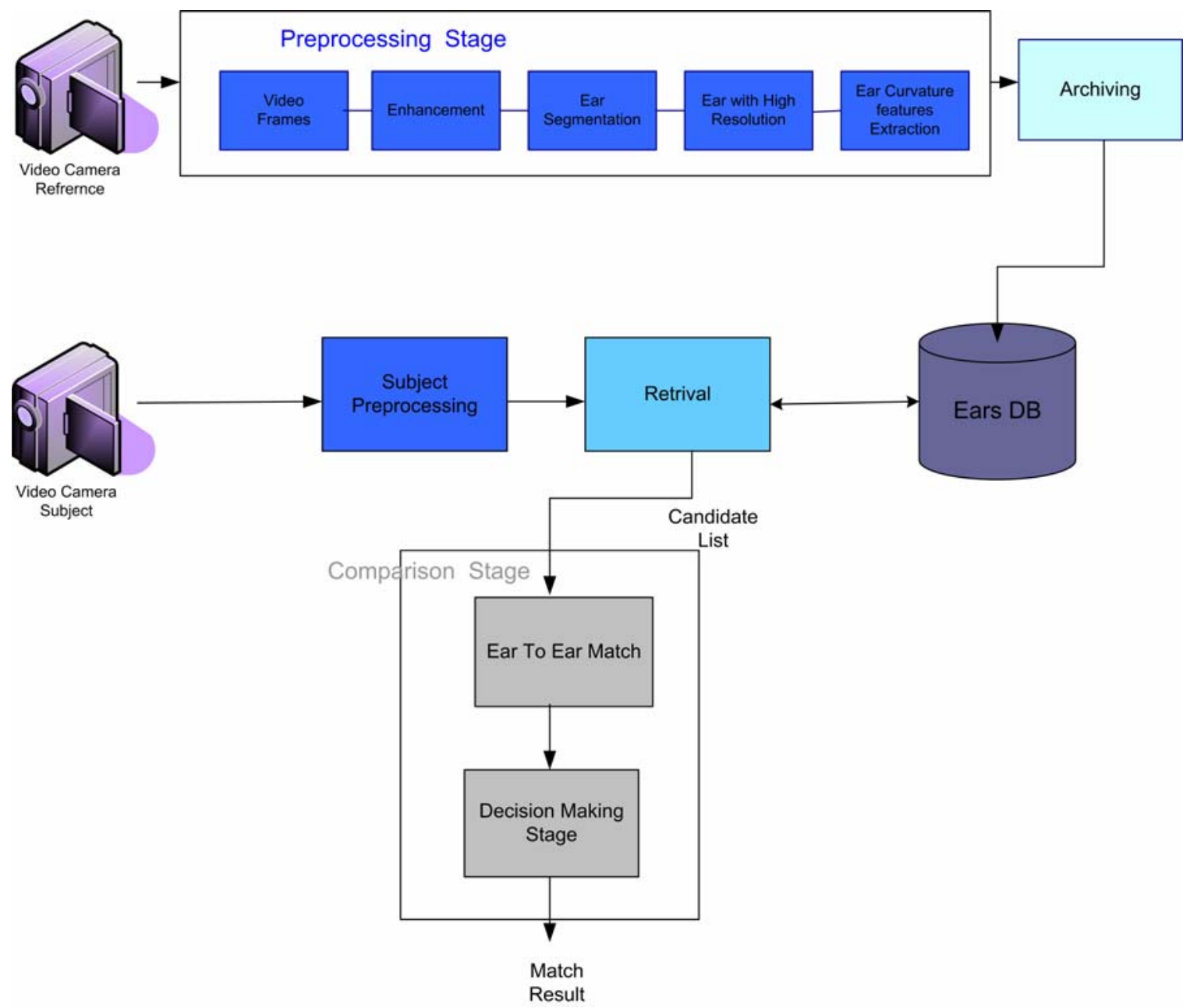

Figure 5: Block diagram illustrating the architecture of AEIS. 


\section{Chapter 2: $\quad$ Problem Statement and Research Objective}

Image segmentation is essential in the biometric and forensic identification process, and it plays a critical role in the most subsequent identification process. The segmentation process depends on modeling the similarity characteristic and common features of the desired object while dealing with variation in intensity, scale, pose, and shape.

However, no single image segmentation technique performs well for all kinds of images; in addition, the performance of various segmentation techniques is not the same and may vary from image to image. Therefore, evaluating the quality of segmentation is critical to measure the efficiency of segmentation algorithms. The evaluation of the segmentation outcomes is typically based on the visual inspection of the images and/or comparison of the segmentation outcome with the ground truth. While the main goal of our research is to develop fully automated system, it is difficult to evaluate the outcome of the segmentation algorithms without human interaction. As a result, the improper segments may affect the accuracy of the identification process and increase the time of the process.

We define the following problems for image segmentation:

1. Develop fully automated segmentation algorithm in order to extract the desired objects from the biometric images.

2. Develop a fully Automated Segmentation Evaluator (ASE) of segmentation outcomes in order to utilize properly segmented images.

\subsection{Dental Image Segmentation}

\subsubsection{Problem Statement}

In the context of ADIS, segmentation is an essential step in the Record Preprocessing component required to identify the extent of teeth comprised in a digital image of a dental radiographic film. At a finer level of detail, segmentation also serves in decomposing a tooth into the crown and root regions. Each segmented tooth represents a Region of Interest (ROI) that contains distinctive features used in the subsequent steps of identification.

\section{Definition 1:}

We define a proper $R O I$ as a rectangular area in the dental film that contains the main parts of the tooth including crown and root. We take into consideration the following: 
1. The ROI includes most of the crown and root of the tooth if there are available in the film as shown in Figure 6.

2. The ROI may contain only the crown when the root is unavailable as shown in Figure $6(d)$.

3. The ROI may include parts of other neighboring teeth or bones as shown in Figure 6 (a), Figure 6 (b).

4. The ROI should not contain the shadow of tooth as shown in Figure 6 (f).

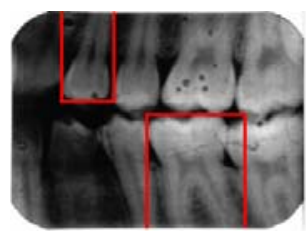

(a)

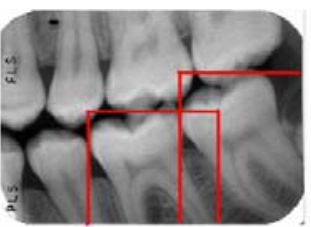

(b)

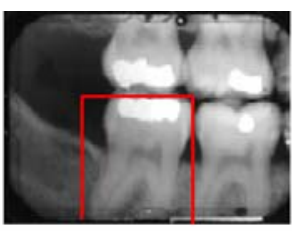

(c)

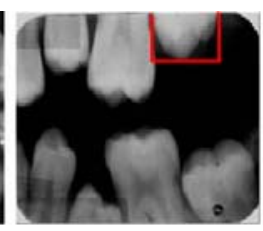

(d)

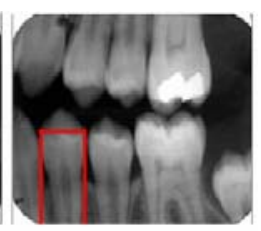

(f)

Figure 6: The rectangular indicates the ROI.

Dental image segmentation will encounter some difficulties that are related to intrinsic factors such as the condition of the human teeth and bone density, or to extrinsic factors such as age of the film and the quality of the scanning machine. Therefore, we define the factors that introduce difficulties in dental image segmentation as follows (i) image blurring (Figure 7 (a) and Figure 7(f)), (ii) fillings (Figure 7(a) and Figure 7(c)), (iii) teeth interfering (Figure 7(a), Figure 7(b), Figure 7(d), Figure 7(f), Figure 7(i), and Figure 7(h)), (iv) image scan quality (Figure 7(e)), (v) the imperceptible difference in intensity between and the teeth (Figure 7(a), Figure 7(d), Figure 7(i), and Figure 7(h)), (vi) dental work (Figure 7(h)).

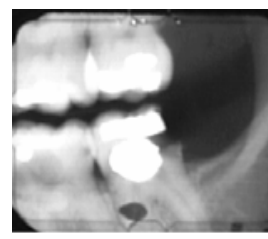

(a)

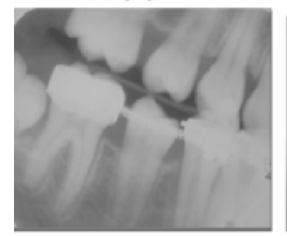

(f)

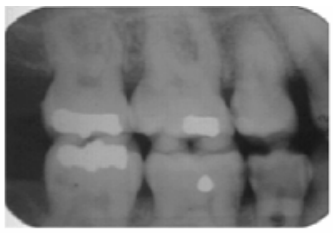

(b)

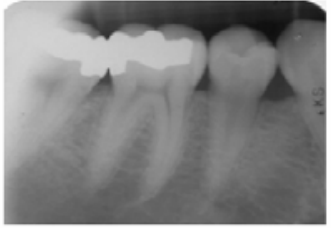

(g)

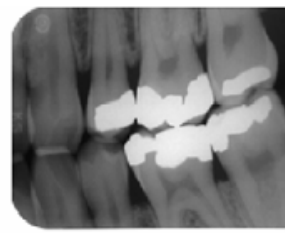

(c)

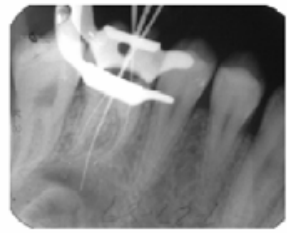

(h)

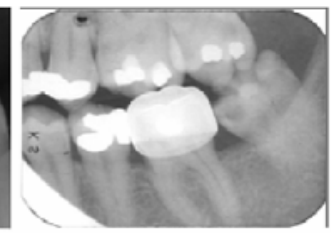

(d)

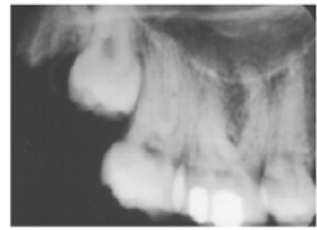

(i)

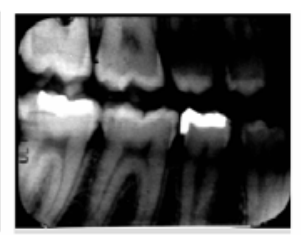

(e)

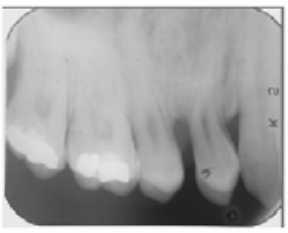

(h)

Figure 7: Examples of difficulties that could face dental image segmentation. 
However, there is hardly any single segmentation technique can perform well for all types of images. In addition, the performance of segmentation techniques is not the same and may vary from image to image. Therefore, segmentation evaluation is critical to measure the efficiency of the segmentation algorithms and to compare their performances.

We define the following problems for dental image segmentation:

- Develop fully automated segmentation algorithm in order to extract proper ROIs from dental radiograph films.

\subsubsection{Research Objectives}

We define the following objectives for dental image segmentation:

1. To automatically extract as many proper ROIs as possible from each film.

2. To operate on bitewing and periapical views. We skip the panoramic views because it does not show fine details unlike the bitewing and periapical views. In addition, the high interference between teeth, or between the teeth and jaws, would lead to a negative effect in identification.

3. Evaluate the performance of proposed segmentation algorithm using a metricsbased object counting approach for empirical assessment of image segmentation, and to compare the performance of our approach with other approaches presented by Jain, Nomair, and Zhou in [8][9][10], respectively.

\subsection{Dental Image Segmentation Automated Evaluation}

\subsubsection{Problem Statement}

The evaluation of the segmentation algorithms will be based on counting the number of correctly detected teeth in a film. In fact, we will visually inspect the outcome of segmentation for each film using a simple rule of object containment within each segment of a given film. While the main goal of ADIS is to develop a fully automated system it is difficult to evaluate the outcomes of the segmentation algorithms without human interaction. The outcomes of the segmentation algorithms could be one of the following:

1. Teeth that are considered as proper ROIs, and it is desirable to pass them through the system as shown in Figure 6. 
2. More than one tooth because of teeth interference, fillings, or high intensity bone structures. These outcomes will lead to over segmented results as shown in Figure 8.

3. Part of the tooth such as crown or root. These results will lead to under segmented results as shown in Figure 9.

4. Background or bones as shown in Figure 10.

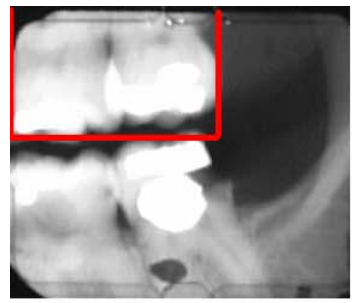

(a)

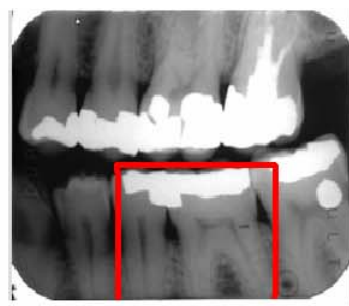

(b)

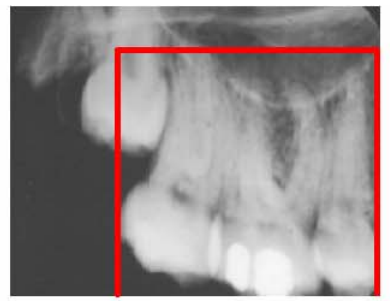

(c)

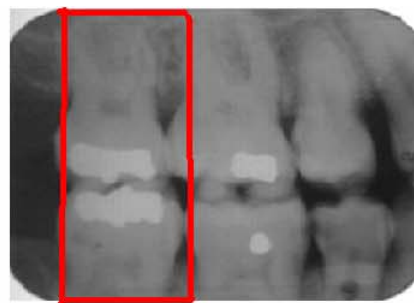

(d)

Figure 8: Examples of over segmentation results, (a) teeth interfering and filings, (b) fillings, (c) teeth interfering, (d) teeth interfering between the upper jaw and lower jaw.

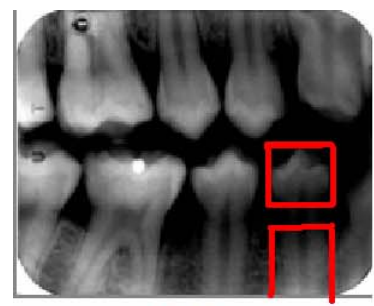

(a)

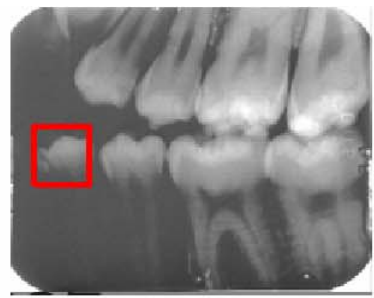

(b)

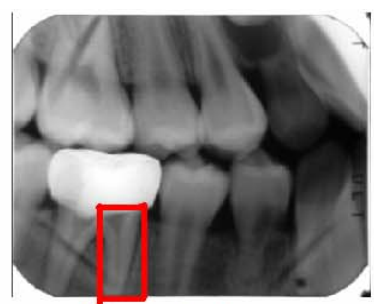

(c)

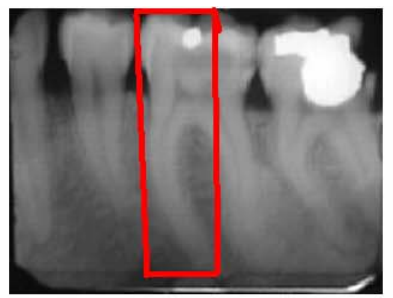

(d)

Figure 9: Examples of under segmentation results.

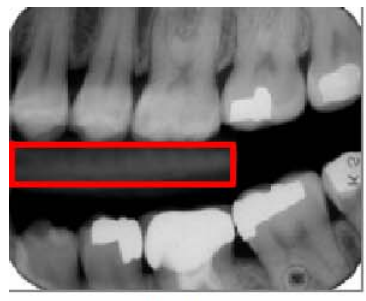

(a)

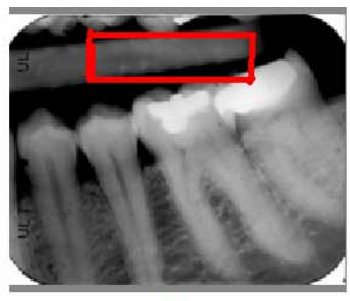

(b)

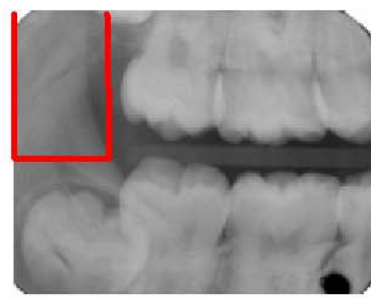

(c)

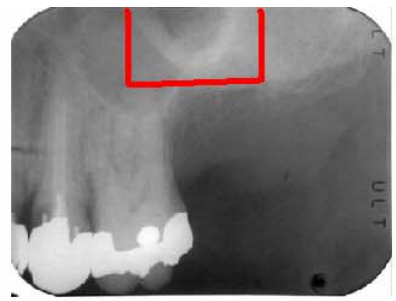

(d)

Figure 10: Examples of segmentation results that related to bones or background.

Passing the over segmented, under segmented, or background outcomes through the identification process may affect the accuracy of the potential matching and the comparison process, and waste computing resources on improper segments. Therefore, it is important to 
automatically evaluate the segmentation outcomes regardless of the technique used for image segmentation.

We define the following problem for the dental image segmentation evaluation:

- Develop an ASE in order to pass only the proper ROIs through the ADIS.

\subsubsection{Research Objectives}

We define the following objectives for ASE of dental image:

1. To automatically classify the outcome of the dental segmentation algorithm as a proper ROI or an improper ROI.

2. ASE will not restrict us to use a specific image segmentation technique nor the best algorithm performance; rather, we will be able to use several segmentation techniques in parallel.

3. Increase the performance of ADIS by reducing the time to process and increasing the segmentation efficiency.

\subsection{Ear Segmentation}

\subsubsection{Problem Statement}

In the context of AEIS, ear segmentation is an essential step in the Preprocessing stage required to extract the ear from multiple views captured from video sequence. Each detected ear represents a Region of Interest (ROI) that contains important features used in the subsequent steps of identification. There are many factors that could affect the ear detection algorithm including variations in scale, dimension, shape and pose, in addition to the effect of hair that covers the ear, earrings, and the beard.

While the main goal of AEIS is to develop a fully automated system, it is difficult to evaluate the outcomes of the segmentation algorithms without human interaction or measuring the difference between outcome image and reference image. Therefore, there is a need to implement an ASE for ear segmentation algorithm in order to recognize the outcome as proper or improper ROI and to avoid human interaction We define the following problems for ear segmentation:

1. Develop fully automated ear segmentation algorithm in order to extract ear from the facial images.

2. Compare the performance of our approach with the one presented in [11] [85]. 
3. Develop fully ASE of ear segmentation outcomes from the facial images.

\subsubsection{Research Objectives}

We define the following objectives for teeth segmentation:

1. Automatically extract the face from the background.

2. Automatically extract the ear from detected face.

3. To operate on multiple views of the subject from the profile view toward the frontal view.

4. Evaluate the performance of our approach and compare it with the one presented in [11][85].

5. To automatically evaluate the outcome of the ear detection algorithm as proper or improper ROI.

6. ASE will not restrict us to use certain ear segmentation algorithm nor the best algorithm performance; rather we will be able to use several segmentation techniques in parallel.

7. Increase the performance of AEIS by reducing the time process and increasing the ear segmentation efficiency. 


\section{Chapter 3: Literature Survey}

\subsection{Dental Identification Systems}

\subsubsection{Semi Automated Dental Identification Systems}

There are many trials to evolve computer- aided postmortem identification systems, but we can consider that CAPMI [12] and WinID [13] are the most popular among these systems. These systems are very developed but none of them can provide us with the desired level of automation, where they need an important role for human interference. As a result, in both CAPMI and WinID feature extraction, coding, and image comparison are passed by hand.

\subsubsection{Assisted Post Mortem Identification system CAPMI}

In 1983 the US Army Institute of Dental Research (USAIDR) developed the Computer Assisted Post Mortem Identification system (CAPMI) in order to enhance the effectiveness of forensic scientists by automatically comparing the reference files and subject files. CAPMI is considered as a sorting tool, not an identification system, and only works effectively when it deals with large numbers of cases [14]. CAPMI generates ranked list which starts with the most likely matches. This list leads forensic odontologists to get the best possible matches of antemortem records to postmortem records. Data entry in CAPMI system can be done either by using a keyboard input or by using an optical mark reader, the dental information associated with each tooth includes:

- Missing tooth

- Unerupted tooth

- Cavity on tooth

- Anomalous condition

- Mesial restoration

- Occlusal restoration

- Porcelain jacket crown

- Facial restoration

- Lingual restoration

- Full coverage crown

- Amalgam restoration
- Stainless steel crown

- Non-metallic restoration ceramic or acrylic/metal

- Distal restoration

- Gold/cast metal restoration

- Temporary restoration, $3 / 4$ crown

- Pointic root canal

- Treated tooth

- Removable partial denture

- Deciduous tooth

- Virgin tooth 
For dental data representation, up to 16 bits of information are used per tooth, for example, if a tooth is present, bit ' 0 ' is set, but if a tooth is absent, then it is reset. To show if a crown is present and the tooth is root filled, the other bits can be used to present the 5 surfaces of the tooth. The following notations are used to depict the features of each tooth:

mesial cavity is entered as (m), a crown is entered as (jmodbp) to refer that all five surfaces are restored (modbp) and a crown is present $(j) .(0)$ refers to the presence of a tooth while (x) to its absence. UL6.mod refers that the Upper Left first permanent molar is present and has restorations in the mesial, occlusal and distal surfaces; while LL3x refers the Lower Left canine is lost. (b: bucal, p: palatal).

The dental information of a subject is compared to the dental information of the candidates by entering the postmortem data for all teeth. The result will be arranged from the most likely match to the least likely match. The comparison product is either: Match, Mismatch, or a possible match. A Match can be found when antemortem and postmortem dental are exactly the same. A Mismatch can be found when antemortem dental condition is different from the postmortem dental condition. A Possible match can be found when the antemortem dental condition is different from the postmortem dental condition but could have evolved into the postmortem condition.

In order to achieve a positive identification, forensic scientists depend on many facts such as: teeth orientation, type of restorative materials, and radiographic appearance. While in CAPMI system these properties are not included because the experiment has proved that these additional data would only increase the processing time and decrease the power of the system and this due to mismatches induced by the subjectivity inherent in the recognition and identification of these entities.

According to study made by the US Army on 7030 Army males, the average person has seven features. For persons with four or more features, $93 \%$ had no other person with the same features. For persons with 7 dental features, the correct match was the peak of the most likely identities list in $95 \%$ of the tests. A correct match was found within the first ten records $100 \%$ of the time. CAPMI is able of comparing and sorting 1200_5000 records per second on most personal computers [6]. 


\subsubsection{WINID}

WinID is a dental computer system that matches missing individuals to unidentified individuals by using dental and anthropometric characteristics in order to class probable matches [13]. There are another information can be inserted into the WinID database, like restored dental surfaces, physical descriptors, and pathological and anthropologic findings. The dental codes utilized in WinID are extensions of the CAPMI codes. In the WinID system we have two kinds of codes, a primary codes and secondary codes, where a dash (-) is placed between the primary and secondary codes. A primary codes and up to five secondary codes can be specified. We can notice that the most investigations in WinID employ only the primary codes because if we are uncertain of a secondary code we can leave the code out. The following tables are listing the primary and secondary codes:

Primary Codes

- M - mesial surface of tooth is restored.

- O - occlusal surface of posterior tooth is restored.

- D - distal surface of tooth is restored.

- F - facial surface of tooth is restored.

- L - lingual surface of tooth is restored.

- I - incisal edge of anterior tooth is restored.

- $\mathrm{U}$ - tooth is unerupted

- $\mathrm{V}$ - non-restored tooth - virgin

- $\mathrm{X}$ - tooth is missing- extracted

- $\mathrm{J}$ - tooth is missing postmortem or the clinical crown of the tooth is not present for examination. Also used for avulsed tooth. The root or an open socket is present, but no other information is available.

- / - no information about tooth is available

Secondary Codes

- A - an anomaly is associated with this tooth. Specifics of the anomaly may be detailed in the comments section.

- $\quad \mathrm{B}$ - tooth is deciduous

- C - crown

- E - resin filling material. 
- G - gold restoration.

- $\mathrm{H}$ - porcelain.

- $\mathrm{N}$ - non-precious filling or crown material. Includes stainless steel.

- $\mathrm{P}$ - pontic. Primary code must be $\mathrm{X}$ to indicate missing tooth.

- $\quad \mathrm{R}$ - root canal filled.

- $\mathrm{S}$ - silver amalgam.

- $\mathrm{T}$ - denture tooth. Primary code must be $\mathrm{X}$ to indicate missing tooth.

- Z - temporary filling material. Also indicates gross caries (used sparingly).

Examples

- MODFL-S mesial occlusal distal facial lingual silver amalgam restoration

- DL tooth has distal lingual restoration

- MODFL-CG gold crown

- MODFL-CHR endodontically treated tooth with porcelain crown

- MI-E mesial incisal resin

- $\mathrm{X}$ tooth missing

- V-B virgin deciduous tooth

- MO-SB mesial occlusal silver amalgam in deciduous tooth

- X-PN missing tooth replaced with non-precious pontic

- X-T missing tooth replaced with denture tooth

- J missing postmortem

- MO-AZ mesial occlusal temporary filling (or caries) on tooth with an anomaly

\subsubsection{CAPMI VS. WINID}

In [15] McGivney emphasized the similarities and differences between CAPMI and WinID. The similarities of the both systems are 1) using the same comparison algorithm, and 2) having the same capability of ranking possible identifications by least number of dental mismatches or most dental hits. On the other hand, only WinID system is able of producing a ranked list of non-dental identifier matches in addition to a list of most restoration hits. The other important advantages of WinID system over CAPMI is its ability to generate the odontograms and to displaying images.

In [16], Lewis and Leventhal proposed a Locator System algorithm that differs from CAPMI and WinID because the Locator System depends on initial screening and classification of AM 
and PM files into 6 categories based on dental work features, such as, the kinds of restored surfaces, attendance of crown and roots, missing teeth. They also stated that the three systems have poor performance with few or no restorations.

In [17], Bowers and cornwellIn compared PM records of two cases to their candidate AM records using operator controlled digital image processing and analysis techniques. For the first AM/PM pair, they set up a common baseline between a tooth- pair by adjusting the orientation of films such that the cemento-enamel junction (CEJ) of the tooth-pair emerges horizontal. In the second AM/PM couple, they chart a smoothed restoration area from the AM tooth to the PM tooth employing an alignment change and conclude that the PM record matches the AM record based on their visual comparison of figures of aligned restorations. Bowers and cornwell refuse the hypothesis that the first PM state matches its candidate AM state by noticing major difference in these measures. They resize the PM tooth such that its (CEJ) measurement agrees with that of the AM tooth, so they measure two age-independent characteristics: the root furcation heights and the distal root divergence angle.

\subsubsection{Automated Dental Identification Systems}

The systems presented in the previous sections have low level of automation and need a lot of human interactions. Therefore; several attempts have been proposed to increase the automation level or to develop fully automated systems for dental identification. In [8], Jain el at proposed a semi automated system for human identification. The system consists of three stages: 1) teeth segmentation, 2) contour extraction, and 3) contour shape matching. This approach only deals with the bitewing and panoramic X-ray dental images and it needs user interaction to choose an initial valley gap point is required to detect the gap valley between the upper and lower jaw.

In [18], Nassar proposed an approach for tooth to tooth matching problem and consider the problem as binary classification problem. They proposed parametric models of class conditional densities, and adaptive strategic technique that used with back propagation to estimate the system parameters. 


\subsection{Ear Identification systems}

Ear structure is a unique characteristic that remains fairly stable over time. Human ear has many variations between individuals, including the various curves and dimensions of the ear [19][20].

It is impossible to prove that an individuals' ear structure is unique, however; several studies have supported this hypothesis. One such study done in California compared over 10,000 ears drawn from a randomly selected sample[4] [21]. This study has shown that in all cases there were no two ears were indistinguishable. Another study examined the difference in ear structures between fraternal and identical twins, who share similarities in many physiological features [22]. It was proven that even though their ear structures were similar, they were still clearly distinguishable. In 1906 Imhofer studied a set of 500 ears and noted that he could clearly distinguish between each ear based on only four features [23]. The results of these studies support the hypothesis that the ear has a unique physiological structure. There is also evidence available to show that ear structure is both stable and permanent. The medical literature reports that ear growth after the first four months of birth is highly linear. It turns out that even though ear growth is proportional, gravity can cause the ear to undergo stretching in the vertical direction. The effect of this stretching is most pronounced in the lobe of the ear and measurements show that the change is nonlinear only in that part. The rate of stretching is approximately five times greater than normal during the period from four months to the age of eight after which it is constant until around the age of seventy when it again increases [22].

Currently, there exists no commercial biometric system to automatically verify the identity of individuals by way of their ear-biometric. However; we present some automated and manual systems that addressed the problem of ear-detection and recognition.

In [4][21], Iannarelli presented personal identification system based on ear features. This system consider one of most popular ear identification system that been introduced in the literature and it has been in use for more than 40 years. The approach is based on extracting 12 features to represent each ear. Each photograph is aligned during development so that the lower tip of a standardized vertical guide on the development easel touches the upper flesh line of the cocha area while the upper tip touches the outline of the antitragus. However; this 
method has some limitations, as all measurements are relative to an origin point, which if not exactly localized, results in all subsequent measurements will be incorrect.

In [22], Chang et. al. presented an approach for biometric recognition based on principle component analysis applied on ear and face images. The results showed that recognition performance is not significantly different between the face and the ear, but the performance significantly improved after using the ear and face results together.

In [24][25], Burge and Burger had used grayscale images of the subject's head in profile images using a CCD camera. They detected the ear by using deformable contours on a Gaussian pyramid representation of the image gradient. Then they made edge extraction, curve extraction, and finally they build a graph model. They have used a novel graph matching based algorithm for authentication, which takes into account the possible error curves.

Chang et al. built multi-modal recognition using both the ear and face [26]. Their research was based on the concept of Eigen faces and Eigen ears using a principal component analysis (PCA) protocol.

Zhang et al. built a hybrid system for ear recognition purpose [27]. This system combines Independent Component Analysis (ICA) and RBF network. The original ear image database is decomposed into linear combinations of several basic images. Then the corresponding coefficients of these combinations are fed up into RBF network instead of an original feature vector comprised of pixel values of grayscale images. The local features extraction of ICA and the adaptability of RBF neural network are then combined.

In [28], Hurley et. al. presented an approach for ear recognition based on force field transformation. They consider the image as an array of Gaussian attractors that work as the source of force field. The extracted features from this technique can be used to create robust and reliable ear biometric system.

In [11] [85], Abdel-Mottaleb et. al. introduced a novel system for ear identification based on the face profile images. The system consists of major steps: 1) ear detection from face profile, and 2) 2-D feature extraction based on force field transformation and recognition based on these features. Testing results applied on 58 query images for 29 subjects showed the efficiency of the system with low time complexity. 


\subsection{Image Segmentation}

Image segmentation is one of the important and challenging issues in image processing field over decades, and it plays critical role in most subsequent image analysis especially in pattern recognition and image matching[29]. The segmentation process is based on recognizing the homogeneity regions in the image as different objects but it is not responsible to define the identity of the object.

There are several techniques proposed for image segmentation. These techniques are devoted to develop fully automated segmentation. However, there is no unified theory for image segmentation and there is hardly any image segmentation technique that performs well in all problems especially when the grey levels of different objects are quite similar. In addition, performance of segmentation technique is greatly affected by noise embedded in images, for example noise due to low resolution and due to poor lighting conditions.

\subsubsection{Medical Imaging Segmentation}

In the following subsections, we will introduce some of techniques that used in medical imaging segmentation.

\subsubsection{Thresholding}

The main goal of thresholding is to divide the image into two classes, the desired objects and undesired objects. A survey of thresholding techniques is available in [30]. Thresholding is effective when the objects in the image have contrasting intensities. Thresholding can be classified into three main categories:

Global thresholding: it utilizes the information of the histogram and may perform iterative thresholding in order to reach the suitable threshold value [9][31] .

Adaptive thresholding: it deals with the spatial information of the pixel in addition to its intensity [32].

Local thresholding: it takes into consideration additional information obtained form edges, or from improving the histogram, or from other variables [33]

However; the performance of the thresholding techniques is highly affected by the initial selected threshold value, and it has limitations in the presence of noise and intensity inhomogeneities [34], or when the objects in the image have very close intensity [35][36]. 
Thresholding may not be used as the only technique in medical image segmentation, but it is used as an essential part in many of the segmentation approaches.

\subsubsection{Region Growing:}

This technique extracts the regions in the images based on grouping the pixels into homogeneous regions by using certain criteria that based on the intensity properties such as edge detection, texture, or/and the type of the available image data. The region growing technique depends on determining a set of seed points from which the region grows based on the specified criteria. Choosing the seeding can be done by user interaction [8]or automatically based on available information such as intensity information [9][10]or texture information[37].

One of the major drawback of this technique is its sensitivity to choosing the initial seed points. Another problem of region growing is choosing the appropriate stopping criteria of growing [37]. Missing some information that the stopping criteria depends on would lead to segmentation error.

However; Region growing is very sensitive to noise [36], and might lead to separate the region into more than one region or lead to merge more than one region. Region growing has large application in field of medical image segmentation. Segmentation examples of medical images are presented in [38][39][40]. Region growing is also used as part of the segmentation process [41][41][42].

\subsubsection{Clustering Algorithms}

Clustering is a supervised method used to partition the pixels in the image into regions that corresponding to different objects. Clustering algorithms do not require training data because they are based on analyzing the property of each object in the image. Clustering is classified into hard clustering where each pixel in the image must belong to one cluster, and soft clustering where pixel can partially belongs to multiple clusters [43]. There are three main algorithms used in clustering[36] i) k-means algorithm that perform iterative computations to group the pixels into clusters based on their distance from the mean of each cluster, ii) fuzzy c-mean algorithm that generalizes the hard c-mean algorithms to allow producing soft clustering, and iii) expectation-maximization. Many approaches based on clustering algorithms have been widely presented in field of medial image segmentation [44][45][46] . 
The major drawback of clustering algorithms is the influence of choosing the initial parameters to the performance of segmentation[36][44][47]. In addition, it doesn't take into consideration the spatial information directly that leads to poor performance in the presence of high level of noise and intensity inhomogeneities [36][48]. To overcome this problem, clustering algorithms are integrated with other techniques such as Markov Random Field (MRF) to perform spatial modeling[48]. On the other hand, the computational cost of MRF is extremely high, and MRF can not keep the topological information [49].

\subsubsection{Neural Network}

Neural Network (NN) are parallel, distributed information structure of processing elements (nodes) that interconnected together in a way similar to neural systems in the human brain. Each node in the network performs an operation to compute its output from its input. The major features of the $\mathrm{NN}$ are 1) its learning capability by modify their behavior in response to their environment where each node adjust its parameters, 2) and generalizing that allows error tolerance and insensitivity to minor changes in the input [50][50]. There are two main types of NN learning algorithms [43]:

1. Supervised learning that used a set of training data. Back propagation is the best known supervised learning algorithms. The main drawback of this method is its bias to patterns similar to ones in the training data. This leads to segmentation error in case of large variety of input images [43].

2. Unsupervised learning that is similar to clustering technique mentioned in the previous section.

However, the main problems of the $\mathrm{NN}$ are determining the network architecture including the number of layers and nodes, lack of transparency, and the long training process in order to achieve acceptable performance [50].

$\mathrm{NN}$ has been widely used in different applications in the medical image segmentation. Examples of these applications are presented in [51][51][52][53].

\subsubsection{Deformable Models}

Deformable model is a powerful technique that combines geometry, physics and approximation theory in order to extract the various shapes of curves, or the surfaces of the desired object. It is also very efficient to locate and track the moving objects. It includes snake, deformable templates, and dynamic contours. A survey in medical image 
segmentation is available in [54]. Deformable model in a static mode depends on setting the initial parameters of the template based on the priori knowledge of the expected shape, placing the template close to desired object, and then performing deformation to fit the curve or surface. The template changes its size and parameters by minimizing the internal force term until it converges to the boundary of the desired object. In a dynamic mode, additional parameter is needed to convey the motion information.

Deformable models have numerous applications in field of medical image segmentation and they have the ability to extract the complex and broad shapes including ultrasound images, X-rays images, CT images , and MR images [54][55][56][57]

Many approaches allow user to initiate the template because deformable models are very sensitive to the initial placement of the templates, and the success of segmentation is highly depending on the good initial position of the template [58]. However; many approaches are presented to decrease the effect of initial parameters.

We summarize the main drawbacks of the deformable models in the following:

1. Adjustment of the parameters in order to minimize the energy term is one of the main problems in the deformable models [59].

2. The performance of the deformable templates is highly affected by occlusion [60].

3. Deformable models are inadequate to deal with variation of the scales, views, and shape [61].

\subsubsection{Mathematical Morphology}

Mathematical morphology (MM) is a topological and geometrical based approach for image analysis, and it is a powerful tool for extracting different shapes and image structures [29]. The shape and the size of the structuring element play an important role in extracting features or objects from the image. Morphological filtering that depends on two major operations [90][62][63], dilation and erosion, has served in a wide range of applications in image segmentation including feature extraction, edge detection, shape representation and description, shape recognition, enhancement, and noise suppression [63]. MM performs enhancement of the image locally rather than globally. It is an excellent tool to extract the dark pixels in the surrounding bright areas and vice versa[64]. MM can work well for segmenting the images that are heavily corrupted with noise. It has the capability to separate 
and eliminate the foreground noise, and the noisy background by applying several MM operators[65].

MM is powerful technique for edge and corner detection even in the presence of noise or in the dark areas [62][66][67]. Many approaches developed for colored images that not only based on RGB component but also on other color component such as HSV [68]. The variety of structuring element shapes and morphological operators make MM robust and powerful technique in field of medical image segmentation including ultrasound images, X-rays images, CT images, and MR images. We will introduce some of mathematical morphology applications in the later sections.

However, MM has some drawbacks when the noise is highly corrupting the images and may lead to oversegmentation [36].

\subsubsection{Radiograph and MR Image Segmentation}

Several radiograph and Magnetic Resonance (MR) image segmentation approaches have been presented in the last decade. In [69] a fully automated technique using Markov random fields was proposed for (MR) images. Noise, inhomogeneity, and structure thickness have bad impact on the performance of the algorithm, and they tend to increase the segmentation error. In [70], Hopfield neural network based on pattern classification using the fuzzy cmeans algorithm was proposed. The computation time and finding the global minimum of the objective function affect the performance. In [71], the segmentation approach is based on analyzing isolable-contour maps to identify coherent regions corresponded to main objects. In [72], the segmentation is based on an improved watershed transform that uses the prior information instead of usual gradient calculations. Although high accuracy was reported in the approaches presented in [71][71][72], user interaction is needed to select interesting regions.

\subsubsection{Dental Image Segmentation}

Although a wide variety of X-ray image segmentation approaches have been presented, there are few researches dedicated to the problem of dental radiograph image segmentation. In [8], Jain and Chen separate the upper jaw and the lower jaw in the bitewing and panoramic dental images by detecting the gap valley between them using the $\mathrm{Y}$-axis projection histogram. Afterwards, the technique isolates each tooth from its neighbors in each jaw by detecting the 
gaps between them using intensity integral projection. Figure 11 shows the steps of segmentation approach proposed by Jain.
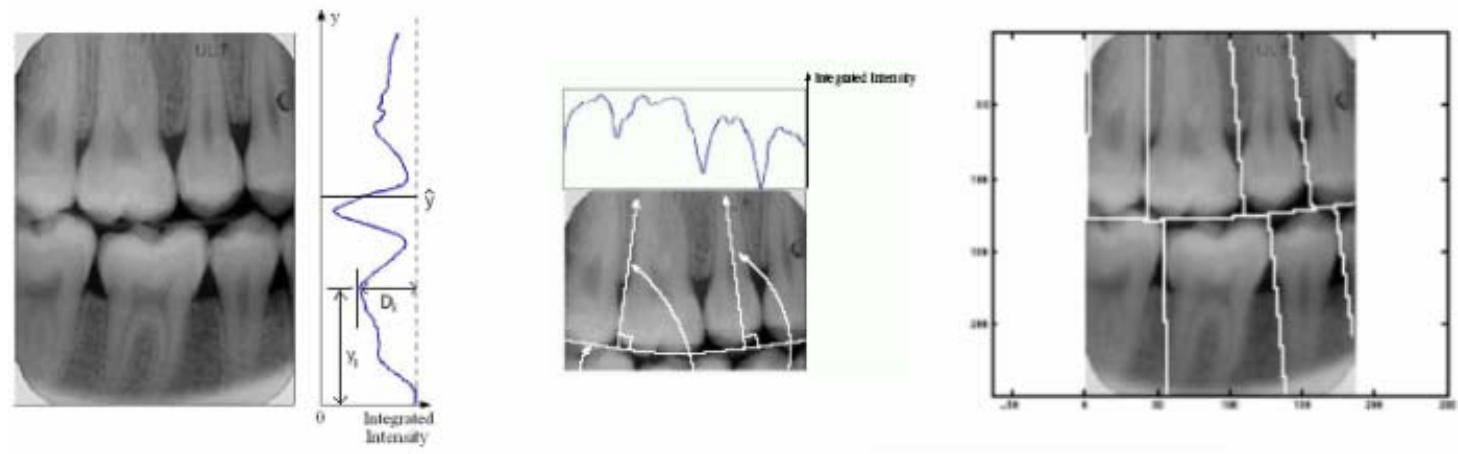

Figure 11: (a) Integral projection on $\mathrm{Y}$ axis, (b) Integral projection of pixels of the upper jaw along the lines perpendicular to the curve of the valley, (c) the segmentation result.

This approach is semi-automated since an initial valley gap point is required to detect the gap valley between the upper and lower jaw. We found from our experiments that the segmentation outcome may vary if we change the position of the selected initial valley gap point. Figure 12 shows two different segmentation results produced by choosing two different initialization points both of which on the valley gap; the choice in Figure 12 (a) leads to perfect segmentation while the choice in Figure 12(b) leads to total segmentation failure.

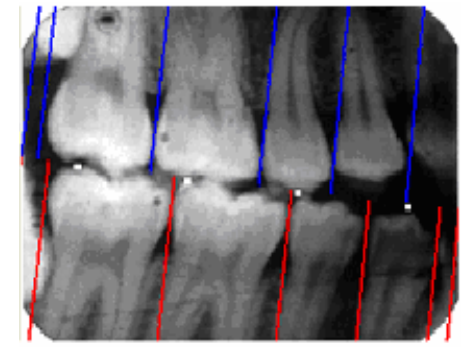

(a)

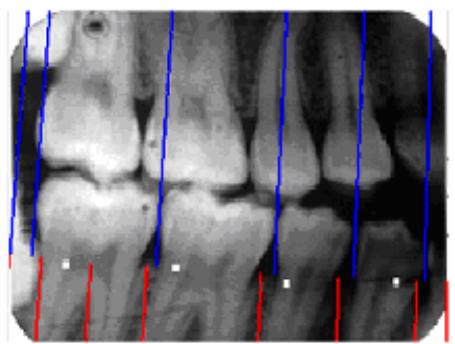

(b)

Figure 12: Two different results for same image. (a) Completely segmented image.(b) Failed segmented image.

In [9], Nomir and Abdel-Mottaleb introduce a fully automated approach for dental x-ray images. The technique depends on applying the following stages (i) iterative threshold to divide the image into two parts, teeth and background, (ii) adaptive threshold in order to increase the accuracy and remove teeth interfering, (iii) horizontal integral projection in order 
to separate the upper jaw from lower jaw, (iv) and finally vertical integral projection in order to separate each individual tooth.

Another fully automated approach for dental x-ray images is introduced by Zhou and AbdelMottaleb [10]. The technique depends on improving the image contrast by applying morphological transformation, and then using window-based adaptive threshold and integral projection to segment the teeth and separate the upper and lower jaw. Figure [13] illustrates some steps of segmentation approach proposed by Mottaleb.
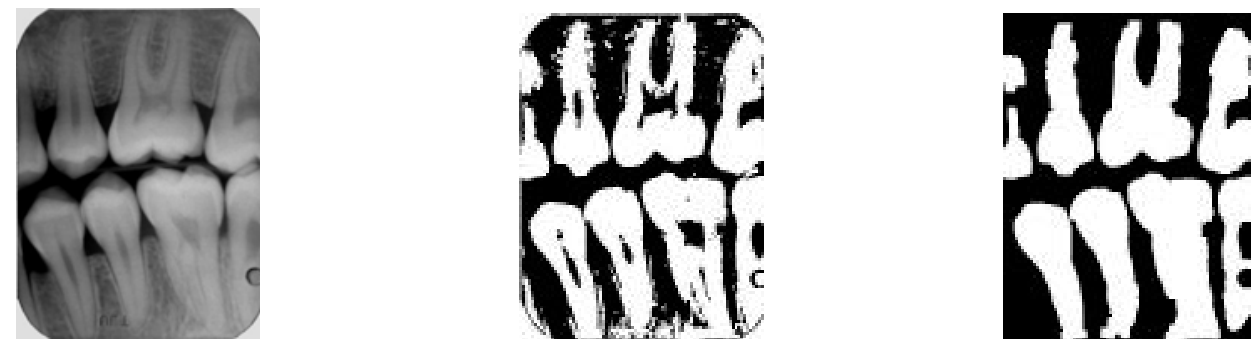

Figure 13: (a) Original Image, (b) the result of MM filtering, (c) the segmentation result.

Table shows a brief comparison between the three algorithms based on underlying principles, type of dental film views they are reported to operate on, and the level of automation they achieve.

Table 1: Summary of comparison between three teeth segmentation algorithms [8][9][10].

\begin{tabular}{|c|c|c|c|}
\hline Algorithm & Principles & Types of views & Is it automated? \\
\hline Jain and Hong & Integral projection & $\begin{array}{c}\text { Bitewing and } \\
\text { Panoramic }\end{array}$ & $\begin{array}{c}\text { No, semi- } \\
\text { automated }\end{array}$ \\
\hline $\begin{array}{c}\text { Nomair and } \\
\text { Abdel-Mottaleb }\end{array}$ & $\begin{array}{c}\text { Iterative and adaptive } \\
\text { thresholding, integral } \\
\text { projection }\end{array}$ & Bitewing only & Yes \\
\hline $\begin{array}{c}\text { Zhou and Abdel- } \\
\text { Mottaleb }\end{array}$ & $\begin{array}{c}\text { Morphology, adaptive } \\
\text { threshold, integral } \\
\text { projection }\end{array}$ & Bitewing only & Yes \\
\hline
\end{tabular}




\subsubsection{Ear Image Segmentation}

In [24][25], Burge et. al. used 300 by 500 profile grayscale images of the subject using CCD camera. They shortly introduced an approach for ear localization based on deformable contour models that presented by affine transformation.

In [11] [85], Abdel-Mottaleb et. al. introduced an approach for ear detection from the profile image that contains the subject's face. The approach consists of three steps: 1) Skin-tone region detection using color and texture thresholding. 2) Edge detection within the region of the skin-tone using canny edge detection and then removing the short edges. 3) Ear detection using ear template that consists of ear's Helix edge.

Chen and Bhanu also used model-based (template matching) based technique for Eardetection [86][86]. The model template is represented by an averaged histogram of shape index. The detection is a four-step process: step edge detection and thresholding, image dilation, connect component labeling and template matching.

Chen and Bhanu then developed another shape model-based technique for locating human ears in side face range images [87] Where the ear shape model is represented by a set of discrete 3D vertices corresponding to ear helix and anti-helix parts. They started by locating the edge segments and grouped them into different clusters which are potential regions containing ears. For each cluster, they register the ear shape model with the edges. The region with the minimum mean registration error is declared as the detected ear region. All approaches presented in [24][25], [11] [85]and, [86][87] used a template matching, where the ear shape is manually predefined. Given an input image, the edges are extracted and then the correlation values with the standard patterns are computed for the different parts/ regions independently. The detection of ear is determined based on the correlation values. This approach has the advantage of being simple to implement.

However, it has proven to be inadequate in the area face detection since it cannot effectively deal with variation in scale, pose, and shape. Multiresolution, multiscale, subtemplates, and deformable templates have subsequently been proposed to achieve scale and shape invariance [88]. 


\subsubsection{Image Segmentation Evaluation}

In [73], Zhang classified the segmentation evaluation methods into two main groups:

analytical, and empirical groups. The analytical methods analyze the segmentation algorithm on basis of its principles, requirements, utilities, complexity, etc. This method is not always able to obtain all of the segmentation algorithm properties because of the limitation of theory for image segmentation [74]. Some of analytical methods are presented in [75][76][77]. The empirical methods evaluate the segmentation algorithms by applying it on test images and then measuring the quality of the segmentation output. Zhang classified the empirical method into two types: goodness method that based on some measurements defined according to human intuition, and discrepancy method that based on comparing the segmentation output with some reference images and computing the difference between them. Some of goodness and discrepancy methods are presented in [78][79][80][81][82][83] respectively. However; goodness method is closer to subjective evaluation rather than objective evaluation of segmentation algorithm, while the discrepancy method depends on the reference images that are not unique in some applications.

In [84], Nassar et. al. present a metrics-based object counting approach for empirical assessment of image segmentation. To evaluate the performance of segmentation algorithm, reference images are used to record the outcome of the experiment in a tabular form as shown in Figure 14.

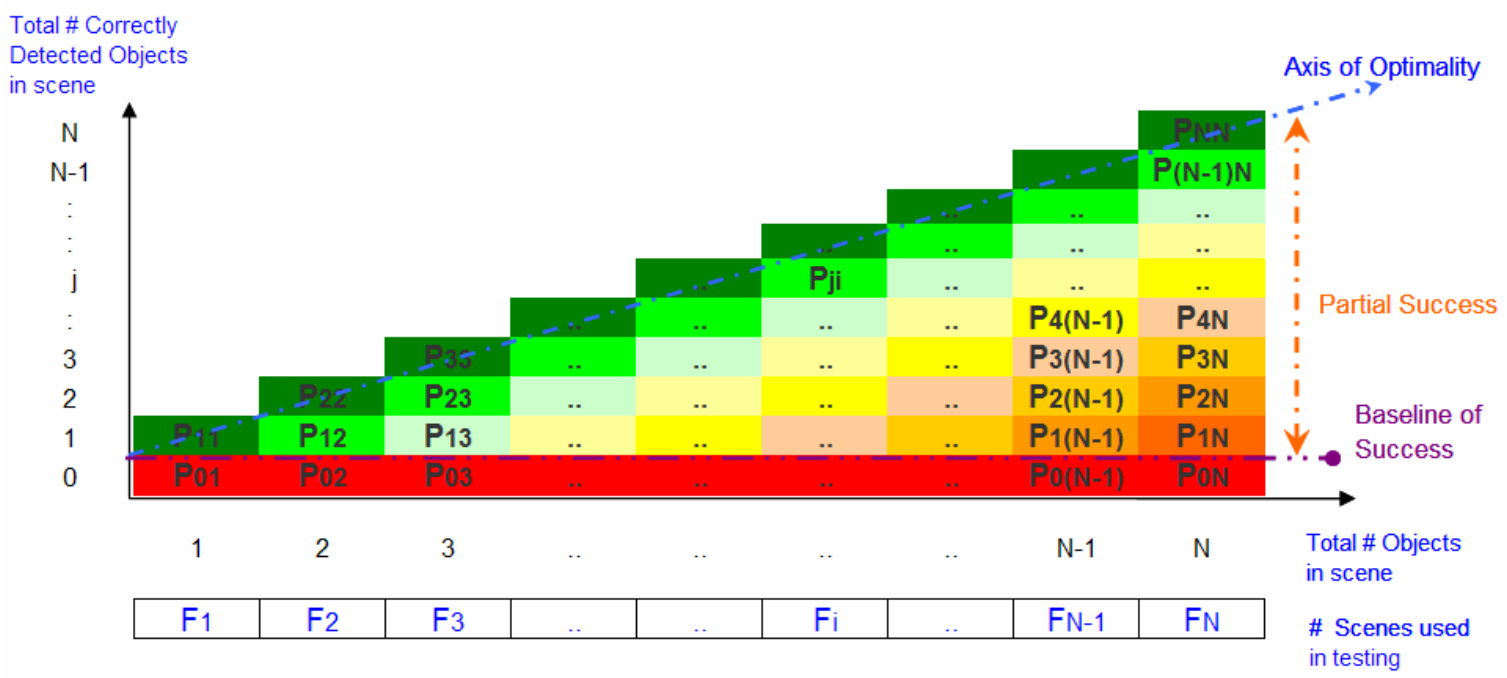

Figure 14: Tabular presentation of segmentation results (adopted from [84]). 
Each cell $P_{j i}$ of the result's table contains the number of instances where the segmentation algorithm correctly detects $j$ objects out of $i$ objects that are present in reference image, with $\sum_{j=0}^{i} P_{j i}=F_{i}$ where $F_{i}$ is the number of reference images that contain exactly $i$ objects. The

results table is used in determining metrics of optimality, sub-optimality and failure based on the relative weights of the main diagonal, the sub-diagonals and the base row respectively, the performance metrics are defined as follows:

Optimality $\equiv 100 * \frac{\sum_{i=1}^{N} P_{i i} F_{i}}{\sum_{i=1}^{N} F_{i}^{2}} \%$
Failure $\equiv 100 * \frac{\sum_{i=1}^{N} P_{0 i} F_{i}}{\sum_{i=1}^{N} F_{i}{ }^{2}} \%$

$\mathrm{m}^{\text {th. }}$ Order Sub-optimality $\equiv 100 * \frac{\sum_{i=1}^{N-m} P_{i(i+m)} F_{(i+m)}}{\sum_{i=1}^{N} F_{i}^{2}} \%$

\subsection{Mathematical morphology}

Mathematical morphology is a topological and geometrical based approach for image analysis, and it is a powerful tool for extracting different shapes and image structures [89]. Morphological filtering [90] has served in a wide range of applications in image processing and analysis. Some of them are in feature extraction, edge detection, shape representation and description, shape recognition, shape smoothing, enhancement, and noise suppression [90]. In this section, I will present the essential morphological operators definitions in the Euclidian setting for gray-scale images. The operators include dilation, erosion, closing, opening, and some morphological filters.

\subsubsection{Gray-Scale Morphology}

\subsubsection{Gray-Scale Dilation}


The dilation of the gray-scale image $f$ and structuring element $g$, with respective Domains $D_{f}$ and $D_{g}$ is defined by:

$$
f \oplus g=D(f, g)=\operatorname{EXTSUP}_{(x, y) \in D f}\left[g_{x, y}+f(\mathrm{x}, \mathrm{y})\right]
$$

Where $g_{x, y}$ represents the translation of an image $g$ by the point $(x, y)$ :

$$
g_{x, y}(s, t)=f(s-t, t-y)
$$

and EXTSUP is defined by:

$$
\begin{aligned}
& \sup \left[f_{k}(t)\right] \quad \begin{array}{l}
\text { if there exists at least one } k \text { such that } f_{k} \text { is } \\
\text { defined at } t \text {, and where the supremum is over } \\
\text { all suck } k
\end{array}
\end{aligned}
$$

$\left[\operatorname{EXTSUP}\left(f_{k}\right)\right](t)=$

$$
\text { undefined if } f_{k}(t) \text { is undefined for all } k
$$

The gray-scale dilation will generate brighter image than the original image, but it will reduce or eliminate the small dark details

\subsubsection{Gray-Scale Erosion}

The erosion of the gray-scale image $f$ and structuring element $g$ is defined by:

$$
f \Theta g=E(f, g)=\operatorname{INF}_{(x, y) \in D g}\left[f_{x, y}+g(\mathrm{x}, \mathrm{y})\right]
$$

Where INF is defined by:

$$
\inf \left[f_{k}(t)\right] \quad \text { if } f_{k}(t) \text { is defined for all } k
$$

$\left[\operatorname{INF}\left(f_{k}\right)\right](t)=$

$$
\text { undefined otherwise }
$$

The gray-scale erosion will generate darker image than the original image, but it will reduce or eliminate the small bright details.

\subsubsection{Gray-Scale Opening and Closing}

We respectively define the dual operations of closing and opening as:

$$
\begin{gathered}
f \bullet g=(f \oplus g) \Theta g \\
f \circ g=(f \Theta g) \oplus g
\end{gathered}
$$

The gray-scale closing will eliminate the peaks in the image and generate an image that has decreased sizes of the small dark details with no appreciable effect on the bright areas, while 
the gray-scale opening will eliminate the holes in the image and generate an image that has decreased sizes of the small bright details with no appreciable effect on the dark areas.

\subsubsection{Closing top-hat transformation}

Closing top-hat transformation is defined by subtracting the image from its morphological closing:

$$
H=(f \bullet g)-f
$$

\subsubsection{Opening top-hat transformation}

Opening top-hat transformation is defined by subtracting the morphological opening of image from itself:

$$
H=f-(f \circ g)
$$

\subsubsection{Morphological gradient}

Morphological gradient is defined by:

$$
\mathrm{G}=(f \oplus g)-(f \Theta g)
$$

\subsubsection{Color Morphology}

There is no straightforward approaches for mathematical morphology for the color images, but we can consider two approaches presented in [91] based on the component wise approach and vector approach.

The component wise approach is based on applying the gray-scale morphology operator on each color of the RGB image:

$$
f \oplus g=\left[f_{r} \oplus g_{r} f_{g} \oplus g_{g}, f_{b} \oplus g_{b}\right]^{T}
$$

The other morphology operators are defined in the same way as above after substituting the dilation denotation with the correspondence operator denotation.

The vector approach is based on considering the color value of each pixel as vector and using reduced ordering functions to define the new vector morphological filtering operations. The vector dilation $\oplus_{v}$ is defined as:

$$
f \oplus_{v} H=\mathrm{a}
$$

Where:

$$
\begin{gathered}
\mathrm{a} \in\{f(r, s):(r, s) \in H\} \\
\mathrm{d}(\mathrm{a}) \geq d(f(r, s)) \forall(r, s) \in H
\end{gathered}
$$


$\mathrm{H}$ is the structuring element, $\mathrm{d}$ is the scalar valued function used for reduced ordering. Similarly, the vector erosion is defined as

$$
f \Theta_{v} H=\mathrm{b}
$$

Where:

$$
\begin{gathered}
\mathrm{b} \in\{f(r, s):(r, s) \in H\} \\
\mathrm{d}(\mathrm{b}) \leq d(f(r, s)) \forall(r, s) \in H
\end{gathered}
$$

\subsubsection{Medical Applications of Mathematical Morphology in Image segmentation}

In [92][93], Leandro et. al. and Zana et.al presented an automated segmentation process for the vessels extraction in the retinal angiographic images. Leandro et. al demonstrate that the mathematical morphology was able to detect very fine structure of the vessels in spite of noise presence. Zana et.al also showed that mathematical morphology is a robust technique for vessel segmentation even for noisy angiographic images.

In [94], Matsopoulos et. al. presented the major medical application using mathematical morphology. They show that morphological filters are robust and powerful tools for medical image processing specially in morphological fusion scheme from different modalities, ultrasound images segmentation scheme, and 3-d reconstruction scheme using morphological edge detection techniques.

In [95], Samarabandu et. al. introduced morphological based methods for fractal analysis of bones Xrays radiographs. These methods present a texture measure of trabecular bones structure that is useful to predict the diseases such as osteoporosis and to classify the textured areas regardless of exposure and magnification.

In [96], Souza et. al. presented an accurate and fast automated approach for extraocular muscles borders detection in orbital CT images using mathematical morphology. This approach is very useful for extraocular muscles segmentation that allow comparisons of graves diseases effect on the eye. In [97], Dogdas et. al. presented new technique of skull segmentation in the MR images using sequence of mathematical morphology filters. They first segment the scalp and the brain from the MR image, and then detect the inner and outer boundaries of the skull using series of thresholding, opening and closing operations.

In [98],Siddiqui et. al. proposed an accurate spot segmentation of DNA microrrary images using mathematical morphology that is used for identification of sequence (gene/gene mutation) and 
determination of gene expression. The results showed that the algorithm is powerful, robust to noise, and it has low time complexity.

\subsection{Principle Component Analysis (PCA)}

Principle component analysis PCA is a statistical method that reduces the number of variables or the dimensionality of the dataset with retaining the maximum amount of variation in it [99]. Because of its ease implementation and reasonable performance [100], PCA has been applied on different application in image processing such as gender classification [101], face recognition [102][103], face detection [104], and teeth classification [110].

Let the vector $\underline{x_{o}}$ represents a dataset of $n d$ dimensional samples $\underline{x_{1}}, \ldots, \underline{x_{n}}$ where the sum of the squared distances between $x_{o}$ and the vectors in the dataset is the minimum. The squared-error criterion can be defined $J_{o}\left(\underline{x_{o}}\right)$ in the following equation:

$$
J_{o}\left(\underline{x_{o}}\right)=\sum_{k=1}^{n}\left\|\left(\underline{x_{0}}-\underline{x_{k}}\right)\right\|^{2}
$$

Let $\underline{m}$ be the mean vector of the given dataset " $\underline{m}=\frac{1}{n} \sum_{k=1}^{n} \underline{x_{k}}$ ". We can write $J_{o}\left(\underline{x_{o}}\right)$ as the following:

$$
\begin{aligned}
J_{o} \underline{\left(x_{o}\right)} & \left.\left.=\sum_{k=1}^{n} \| \underline{\left(x_{0}\right.}-\underline{m}\right)-\underline{\left(x_{k}\right.}-\underline{m}\right) \|^{2} \\
& \left.=\sum_{k=1}^{n} \| \underline{\left(x_{0}\right.}-\underline{m}\right)\left\|^{2}-2 \sum_{k=1}^{n}\left(\underline{x_{0}}-\underline{m}\right)^{T}\left(\underline{x_{k}}-\underline{m}\right)+\sum_{k=1}^{n}\right\| \underline{\left(\underline{x_{k}}-\underline{m}\right) \|^{2}} \\
& \left.=\sum_{k=1}^{n}\left\|\left(\underline{x_{0}}-\underline{m}\right)\right\|^{2}-2\left(\underline{x_{0}}-\underline{m}\right)^{T} \sum_{k=1}^{n}\left(\underline{x_{k}}-\underline{m}\right)+\sum_{k=1}^{n} \| \underline{\left(x_{k}\right.}-\underline{m}\right) \|^{2}
\end{aligned}
$$

The second term is equal to zero because:

$$
\begin{aligned}
-2\left(\underline{x_{0}}-\underline{m}\right)^{T} \sum_{k=1}^{n}\left(\underline{x_{k}}-\underline{m}\right) & =-2\left(\underline{x_{0}}-\underline{m}\right)^{T}\left(\sum_{k=1}^{n} \underline{x_{k}}-\sum_{k=1}^{n} \underline{m}\right) \\
& =-2\left(\underline{x_{0}}-\underline{m}\right)^{T}\left(\sum_{k=1}^{n} \underline{x_{k}}-n \underline{m}\right)
\end{aligned}
$$




$$
=-2\left(\underline{x_{0}}-\underline{m}\right)^{T}\left(\sum_{k=1}^{n} \underline{x_{k}}-n \frac{1}{n} \sum_{k=1}^{n} \underline{x_{k}}\right)=-2\left(\underline{x_{0}}-\underline{m}\right)^{T}(0)=0
$$

Since $\underline{m}=\frac{1}{n} \sum_{k=1}^{n} \underline{x_{k}}, J_{o}\left(\underline{x_{o}}\right)$ can be written as:

$$
\begin{aligned}
J_{o}\left(\underline{x}_{o}\right) & =\sum_{k=1}^{n}\left\|\left(\underline{x_{0}}-\underline{m}\right)-\left(\underline{x_{k}}-\underline{m}\right)\right\|^{2} \\
& =\sum_{k=1}^{n}\left\|\left(\underline{x_{0}}-\underline{m}\right)\right\|^{2}+\sum_{k=1}^{n}\left\|\left(\underline{x_{k}}-\underline{m}\right)\right\|^{2}
\end{aligned}
$$

$J_{o}$ is minimized when $\left(\underline{x_{o}}=\underline{m}\right)$, and the second term is independent of $\underline{x_{o}}$.

The sample mean is a zero-dimensional representation of the data because it will project the data into single point in $\mathbf{R}^{d}$, and it does not give us any of the variability in the data.

$J_{o}(\underline{m})=\sum_{k=1}^{n}\left\|\left(\underline{x_{k}}-\underline{m}\right)\right\|^{2}=\sum_{k=1}^{n}\left(\left\|\underline{x_{k}}\right\|^{2}-2 \underline{x_{k}} \underline{m}+\|\underline{m}\|^{2}\right)$

If we derive $J_{o}$ with respect to the mean vector we will get

$\sum_{k=1}^{n}-2 \underline{x_{k}}+\sum_{k=1}^{n} 2 \underline{m}=-2 n_{-} \underline{m}+2 n_{-} \underline{m}=0$

Therefore, if we project the data onto a line running through the sample mean vector, we will get one dimensional representation of the data. The equation of the line is written as:

$\underline{x}=\underline{m}+a \underline{e}$

Where $\underline{e}$ is a unit vector in the direction of the line, and a is a scalar that represents the distance between the mean vector and any point $\underline{x}_{\underline{x}}$ in the data. By representing $x_{k}$ by $\underline{m}+a_{k} \underline{e}$, we find the optimal coefficient $a_{k}$ by minimizing the squared error criterion function 


$$
\begin{aligned}
j\left(a_{1}, a_{2}, \ldots a_{n}, \underline{e}\right) & =\sum_{k=1}^{n}\left\|\left(\underline{m}+a_{k} \underline{e}\right)-\underline{x_{k}}\right\|^{2} \\
& \left.=\sum_{k=1}^{n} \| a_{k} \underline{e}-\underline{\left(x_{k}\right.}-\underline{m}\right) \|^{2} \\
& \left.=\sum_{k=1}^{n}\left\|a_{k} \underline{e}\right\|^{2}-2 \sum_{k=1}^{n}\left(a_{k} \underline{e}\right)^{T} \underline{\left(x_{k}\right.}-\underline{m}\right)+\sum_{k=1}^{n} \underline{\| x_{k}}-\underline{m} \|^{2} \\
& \left.=\sum_{k=1}^{n} a_{k}^{2}\|\underline{e}\|^{2}-2 \sum_{k=1}^{n} a_{k} \underline{\underline{e}} \underline{\left(x_{k}\right.}-\underline{m}\right)+\sum_{k=1}^{n}\left\|\underline{x_{k}}-\underline{m}\right\|^{2}
\end{aligned}
$$

We minimize the squared error criterion function by partially differentiating it with respect to $a_{k}$, and setting the derivative to zero.

$a_{k}=\underline{e}^{T}\left(x_{k}-\underline{m}\right)$

Another important point is to find the best direction e for the line and that can be resolved through scatter matrix $\mathrm{S}$ defined in the following equation:

$$
S=\sum_{k=1}^{n}\left(\underline{x_{k}}-\underline{m}\right)\left(\underline{x_{k}}-\underline{m}\right)^{T}
$$

The scatter matrix $\mathrm{S}$ is $\mathrm{n}-1$ times the sample covariance matrix.

$$
\begin{aligned}
J_{1}(\underline{e}) & =\sum_{k=1}^{n} a_{k}^{2}-2 \sum_{k=1}^{n} a_{k}^{2}+\sum_{k=1}^{n}\left\|\left(\underline{x_{k}}-\underline{m}\right)\right\|^{2} . \\
& \left.=-\sum_{k=1}^{n}\left[\underline{e}^{T} \underline{\left(x_{k}\right.}-\underline{m}\right)\right]^{2}+\sum_{k=1}^{n}\left\|\left(\underline{x_{k}}-\underline{m}\right)\right\|^{2} \\
& \left.=-\sum_{k=1}^{n} \underline{e^{T}} \underline{\left(x_{k}\right.}-\underline{m}\right)\left(\underline{x_{k}}-\underline{m}\right)^{T} \underline{e}+\sum_{k=1}^{n}\left\|\left(\underline{x_{k}}-\underline{m}\right)\right\|^{2}
\end{aligned}
$$




$$
=-\underline{e}^{T} S \underline{e}+\sum_{k=1}^{n}\left\|\left(\underline{x}_{k}-\underline{m}\right)\right\|^{2}
$$

Vector $\underline{e}$ maximizes $\underline{e}^{T} S \underline{e}$ and at the same time minimizes $J_{1}$

To maximize the $\underline{e}^{T} S \underline{e}$, we use the method Lagrange multipliers. Using $\lambda$ as the undetermined multiplier.

$u=\underline{e}^{T} S \underline{e}-\lambda\left(\underline{e}^{T} \underline{e}-1\right)$

By differentiating the equation with respect to e and set to zero we get:

$\frac{d u}{d \underline{e}}=2 S \underline{e}-2 \lambda \underline{e}=0 \Rightarrow S \underline{e}=\lambda \underline{e}$

More specifically, and since $\underline{e}^{T} S \underline{e}=\lambda \underline{e}^{T} \underline{e}=\lambda$ we can conclude the following:

To maximize $\underline{e}^{T} S \underline{e}$, we select the eigenvector corresponding to the largest eigenvalue of the scatter matrix. As a result, to find the best one dimensional project of the data, we project the data onto a line through a sample mean in the direction of the eigenvector of the scatter matrix having the largest eigenvalue.

\subsubsection{Principle Component Analysis Applications}

In [105], Moon et. al. introduced a generic modular PCA algorithm for face recognition that allows us to systematically change the components and determine the effect of these changes in the performance of algorithm. It consists of three step, normalization, PCA projection, and recognition. The experiment results show that 1) the studies should be applied on large range of image qualities and for more than type of facial images 2) implementation details of the algorithm could have highly effect on the performance and the results of the algorithm.

In [106][107], Turk et. al. presented an Eigen face approach for human face recognition that tracks the head in the image, and then recognize the person according to its characteristic. 
They took the fact that faces have upright views and can be described by a small set of 2-D characteristic views.

The idea behind the approach is to project the face images onto a feature space that spans the variations in the known face images. The variations of face features which extracted from a training set of faces are known as eigenfaces. After that, face recognition is achieved by comparing the weights obtained form projection with those of the known faces. The experimental results show that the eigenface approach is a robust and accurate technique for face recognition even with a substantial unknown rejection rate and its time complexity is reasonable.

In [108], Pentland et. al. proposed view-based multiple observer eigenspace technique for face recognition. They demonstrated an accurate visual recognition using a database of 3000 individuals.

In [109], Rajagopalan et.al. proposed an algorithm for face recognition in the presence of clutter based on PCA. They stated that the traditional eigenface recognition method has poor quality in the presence of background clutter. Therefore they created an eigenbackground space and derived a classifier to recognize the non-face patterns from the faces. The testing results showed that the algorithm has robust performance in the real situation where the cluttered background is presented.

In [110], Nassar presented an automated approach of teeth classification into incisors, canines, premolars, and molar based on PCA and string matching technique. The first step of the approach is capturing the most common features of each of the teeth classes using four image subspaces that defined as eigen teeth. Nassar chose the crown area of the teeth samples to construct the image subspace and resized them into 32X32 pixels based on the bicubic interpolation scheme. He vertically flipped the maxillary plane exemplars to reduce the complexity of the problem.

The next step is normalizing the view of the segmented teeth by: 1) detecting the edges (canny edge detection), groping them based on the connectivity, and eliminating the short edges, 2) estimating the orientation of the edge points, classifying tem as vertical and horizontal, and computing the histogram of the orientations of the vertical points, 3) rotating 
the input image such that side surfaces of the segmented tooth becomes vertical, 4) confining the segmented tooth to square area, and resize it to $32 \mathrm{X} 32$.

The next step is to project the normalization view into the four subspaces, Incisors, Canines, Premolars, and Molar subspaces, and to reconstruct the tooth in the four subspaces. After that Nassar initially classified the tooth which corresponds to the least energy discrepancy between the reconstructed tooth and its approximations. The last step of teeth classification is to validate the correct the initially classified tooth based on string matching. Testing results showed that $75 \%$ of teeth are initially classified correctly; $86 \%$ are classified correctly after validation step. 


\section{Chapter 4: Contribution}

Segmentation step is essential in the biometric identification process. Although the biometric systems depend on capturing the differences between individual characteristic, the segmentation depends on capturing the similarity characteristic and common features of the individuals.

In our research, we develop segmentation algorithm based on mathematical morphology that could be used for different kind of biometric systems and apply it on the dental radiograph films and facial images to extract the teeth and ear respectively. The philosophy behind choosing mathematical morphology is its powerful in dealing not only with the structure and the shape of the desired object but also with intensity of that object.

Mathematical morphology has variant of powerful filters that have the ability to automatically detect the dark pixels on surrounding brighter pixels and vice versa. In addition it locally performs filtering taking the shape, intensity, and the dimension of the desired object in its consideration. Mathematical morphology can be applied on the visible images such as facial images and fingerprints, and invisible image such as X-ray and MR images. We face many factors that introduced difficulties in teeth segmentation and ear detection. Mathematical morphology has the ability to overcome most of these difficulties. These factors are:

1. The lack of a unified standard for scanning and digitization of dental radiographic films.

2. Dental work on the dental radiograph films.

3. High intensity of the bones

4. Image blurring.

5. Teeth interfering and interfering between the ear and other object such as hair, beard, and earrings.

We conclude the new contributions of our work in the following:

1- Develop fully automated segmentation algorithm based on mathematical morphology for biometric systems.

2- Apply the approach on the dental radiograph films and facial images to extract teeth and ear respectively 
3- The approach deals with periapical dental images, while all other approaches for teeth segmentation do not deal with the periapical images.

4- Develop fully ASE of the segmentation outcomes without human interaction. 


\section{Chapter 5: Approach for Segmentation Algorithm}

Automated object segmentation in the biometric systems plays a critical role in leading the process of object to object matching. Segmentation output reflects the position of the object in the image that helps to match related objects in the image.

In this Chapter, we present an approach for fully automated segmentation of some of the biometric systems that relies on Mathematical Morphology, Thresholding, and connectivity properties. We apply this approach on segmenting the teeth from bitewing and periapical images in the dental radiograph films, and the ear from the facial images. After that we evaluate the performance of proposed segmentation algorithm using metrics-based object counting approach for empirical assessment of image segmentation, and compare the performance of our approaches with other approaches presented by Jain, Nomair, and Zhou in [8][9][10] respectively.

\subsection{Overview}

We classify the main objects of the biometric images according to their intensity and shape, and then we use series of morphology filtering operations to ease the segmentation process. Choosing the morphology filters is based on the type of desired object, its intensity, and its shape. After that we apply thresholding on the filtered image in order to obtain binary image, and then we analyze the connected components obtained from thresholded image according to their geometric properties in order to obtain the proper ROIs. Figure 15 shows the main steps of the segmentation algorithm.

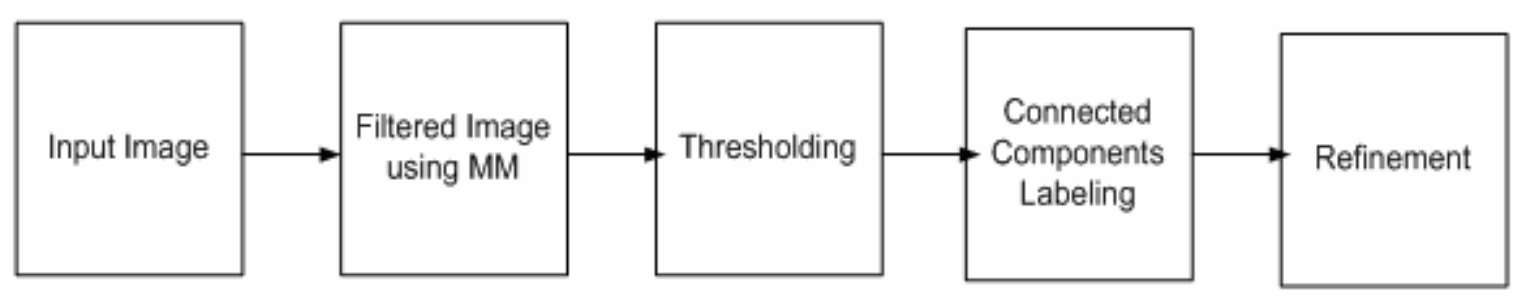

Figure 15: Main stages of proposed segmentation algorithm. 


\subsubsection{Filtering Using Mathematical Morphology:}

Mathematical morphology has variant of powerful filters that have the ability to automatically detect the dark pixels surrounded by brighter pixels and vice versa. In addition, it locally performs filtering, taking the shape, intensity, and the dimension of the desired object in its consideration. The main goal of this step is to emphasize the desired object and to suppress the other objects. The morphological filter is chosen based on the intensity of the desired object with consideration of surrounding object intensity, while the structuring element is chosen based on the shape and dimension of the desired object.

\subsubsection{Thresholding}

In order simplify the image analysis; we produce the binary image from the filtered image produced in the previous stage by using the thresholding. Choosing the thresholding process depends on the features of the desired object.

\subsubsection{Connected components labeling}

We group pixels of the threshold image based on their connectivity and assign them labels that identify the different connected components. The connected component labeling is based on 8-connected neighborhood and 4-connected neighborhood algorithms to group the pixels and to label them.

\subsubsection{Refinement}

The purpose of refinement is to analyze the connected components based on geometric properties (area, position and dimension) and then to eliminate the improper ROIs. We define the position, area, and dimension of a region by:

1- The position is defined by using the position of the centriod of the region.

2- $\quad$ The area is defined by the number of pixels of the region.

3- The dimensions are defined by the width and height of the region.

The rules used for refinement differ according to desired object properties.

We apply the previous stages on two different biometric systems; one of them is using dental $\mathrm{X}$-ray images in order to segment the teeth, and the other one is using facial images with different views in order to segment the ear. 


\subsection{Teeth Segmentation from Dental $x$-rays Images}

We define three main classes of objects in the X-ray dental images, teeth that map the areas with "mostly bright" grayscale and semi rectangular shape, bones that map areas with "midrange" grayscale with no certain shape, and background that maps "dark" grayscale with no certain shape. In the following subsections, we will present in details the main stages of extracting teeth from dental radiograph.

\subsubsection{Filtering in the Dental Images:}

We start with detection of the gap valley between the upper jaw and the lower jaw, bones between the teeth, teeth interference, and the gaps between the teeth. We define intrinsic noise as the combination of these factors. Detection and suppression of the intrinsic noise help to emphasize the teeth with respect to the background. Figure 16 shows three samples of grayscale line profiles; the upper horizontal line profile illustrates the bones between the teeth, the lower horizontal line profile shows the gap between the teeth, while the vertical line profile illustrates the gap valley. Closing top hat transformation, which is defined by subtracting the image from its morphological closing, provides an excellent tool for detecting pixels that are dark on the surrounding bright areas, and it locally performs suppression of teeth and emphasizes intrinsic noise.

The grayscale line profiles of the closing top hat transformed image represented in Figure 17 show the emphasized intrinsic noise. Figure 18 shows an example of a dental film before and after removal (subtraction) of intrinsic noise. We use a rectangular structuring element with dimensions $[w / 4, h / 2]$ for bitewing images, and with dimensions $[w / 3,2 * h / 3]$ for periapical images, where $w$ and $h$ are the width and the height of the image respectively. Our choice of these structuring elements is based on an experimental study on a set of 20 bitewing views and 20 periapical views of different sizes and qualities. 

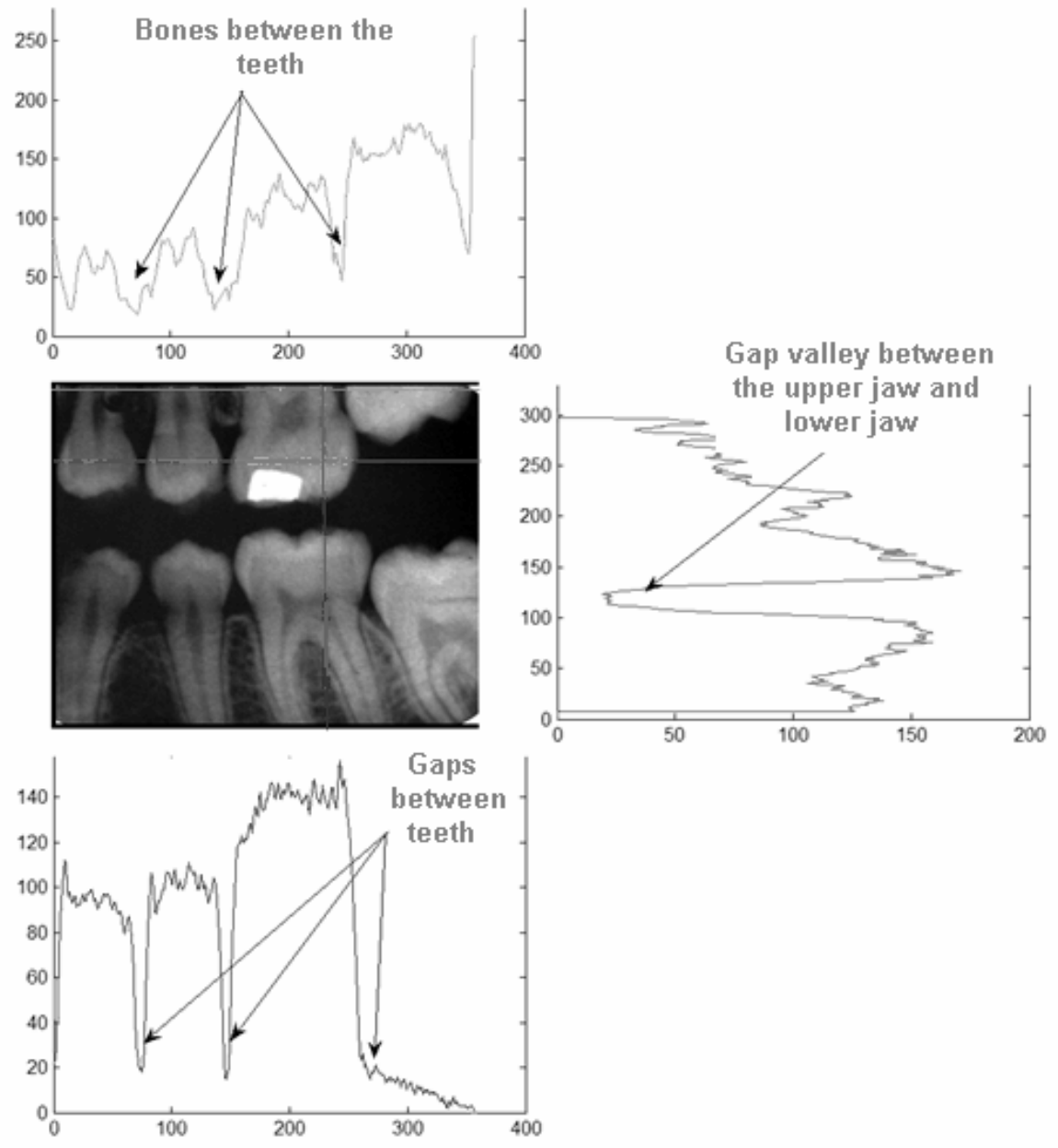

Figure 16: Grayscale line profiles of the input image, the upper horizontal line profile illustrates the bones between the teeth, the lower horizontal line profile shows the gap between the teeth, while the vertical line profile illustrates the gap valley. 

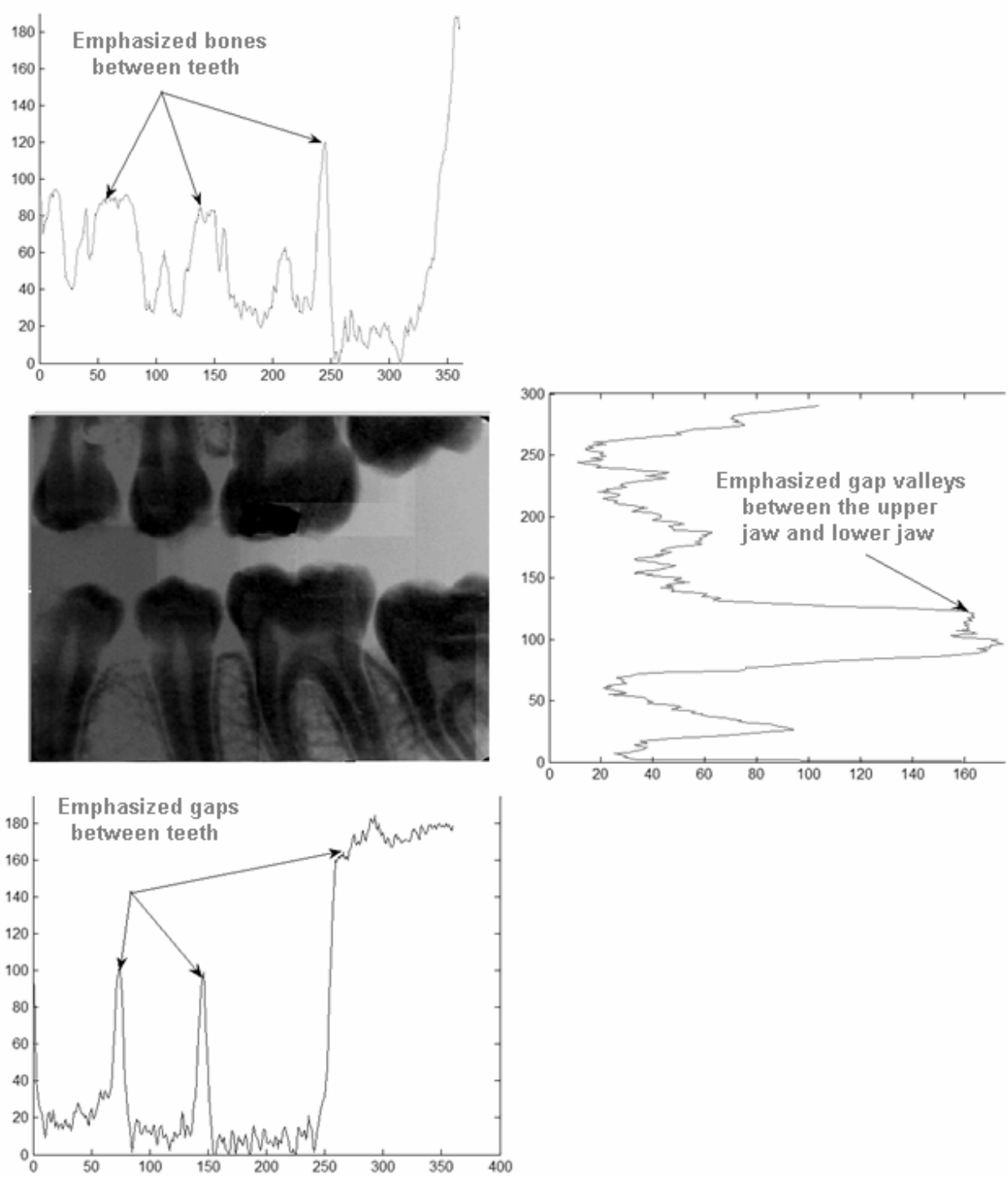

Figure 17: Grayscale line profiles of the closing top hat transformed image that are corresponded to the same line profiles in Figure 16 


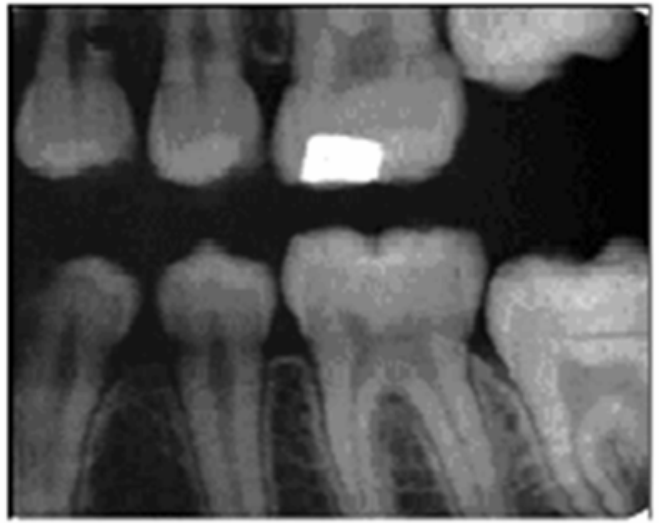

(a)

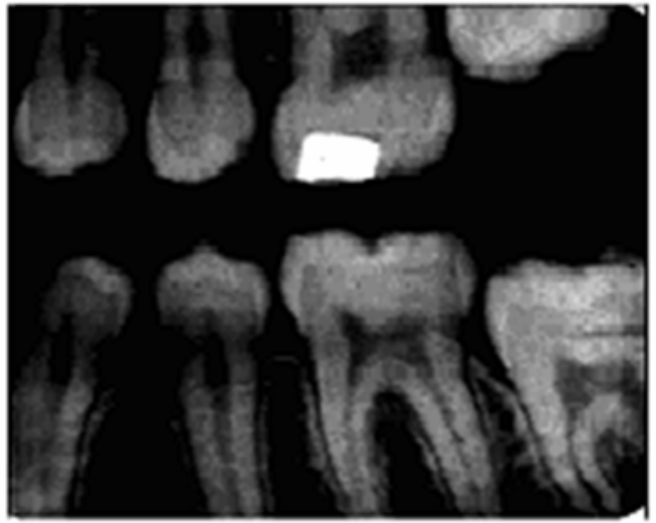

(b)

Figure 18: (a) Original image. (b) Filtered image.

\subsubsection{Thresholding in the Dental Images}

After reducing the noise effect, we use threshold operation to separate the desired teeth from the background and the remaining noise. In many of the dental radiographs, we notice presence of a shading effect that manifests as a gradient of image brightness from side to side as shown in the horizontal grayscale line profiles of the image in Figure 16. Therefore, choosing a single threshold value is not preferable because it may result in missing information pixels. The cumulative histogram of the filtered image, which contains the percentage of pixels below a certain grayscale level, gives the percentage of pixels that are set to zero after reducing the noise. In our example, around $50 \%$ of the image pixels are set to zero as shown in Figure 19. According to an experimental study applied on a set of 100 dental images, we found that taking three threshold values would produce the most number of the proper ROIs in the following stages. The threshold values $T_{1}, T_{2}$, and $T_{3}$ fall between the mean value of the filtered image and zero, where: $T_{1}=$ mean (Filtered Image), $T_{2}=0.66$ $T_{1}, T_{3}=0.33 T_{1}$.

Images in Figure 20 (a)-(c) show the three different results obtained from thresholding 


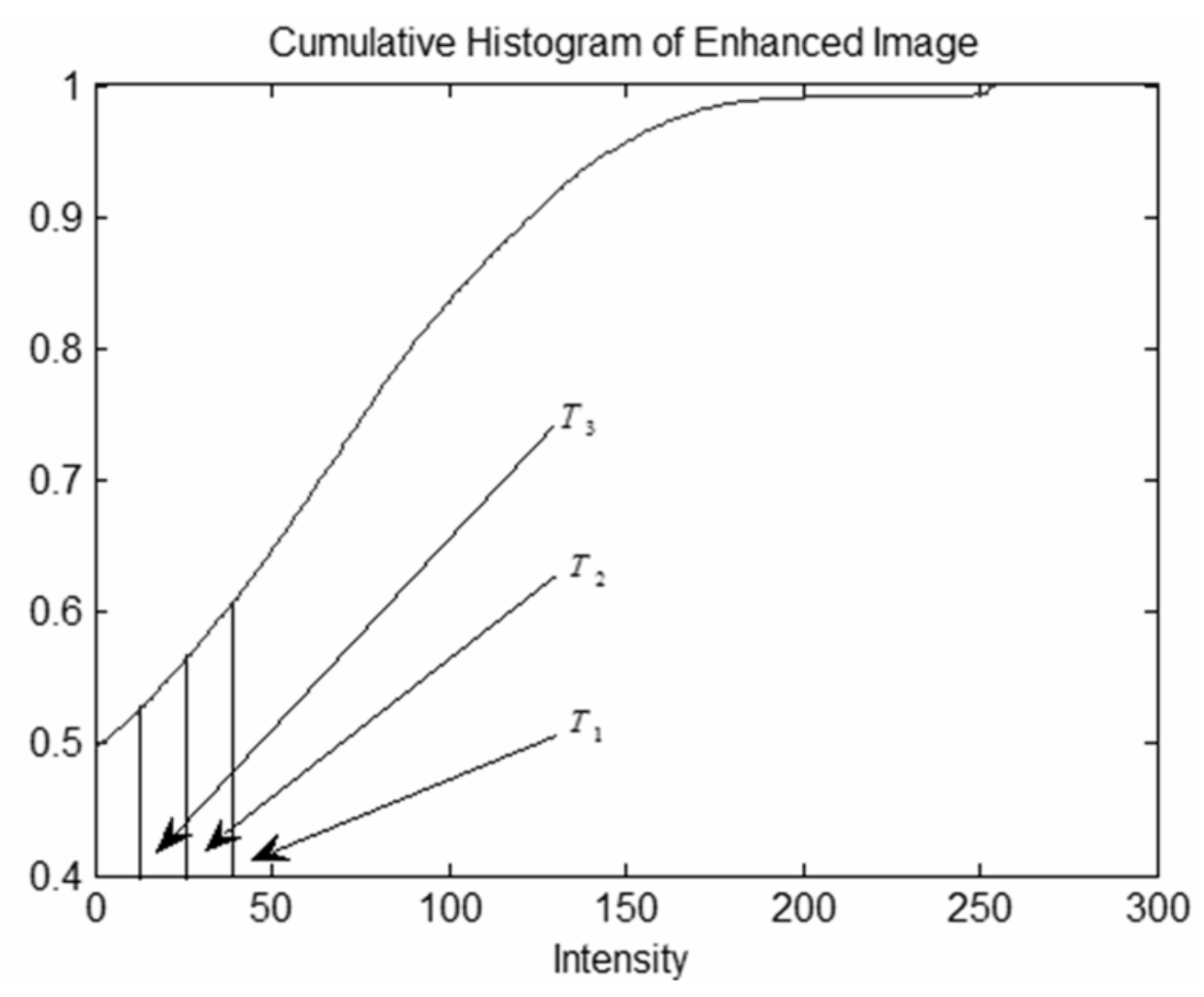

Figure 19: Cumulative histogram of the filtered image.

\subsubsection{Connected Components Labeling in the Dental Images}

We group pixels of the threshold image based on their connectivity and assign them labels that identify the different connected components. Images in Figure 20 (d)-(f) show the results of the connected components labeling for each thresholded image shown in Figure 20 (a)-(c) respectively. The connected components in the dental images can be attributed to (i) teeth that are considered as ROIs, (ii) more than one tooth because of teeth interference, fillings, or high intensity bone structures, (iii) background or bones, (iv) part of the tooth such as crown or root.

\subsubsection{Refinement in the Dental images}

We analyze the connected components based on their geometric properties including area and dimension, and then we only pass the objects that satisfy the refinement rules. Table 2 shows the rules used for refinement based on an experimental study applied on 30 images. 


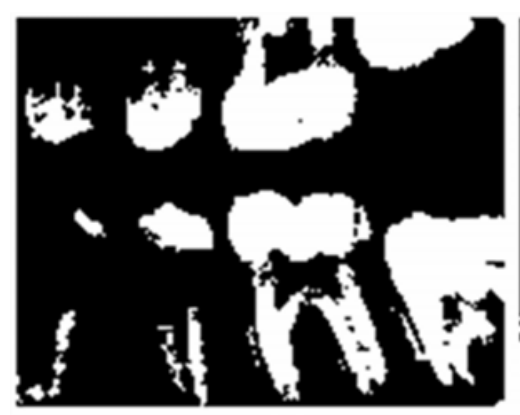

(a)

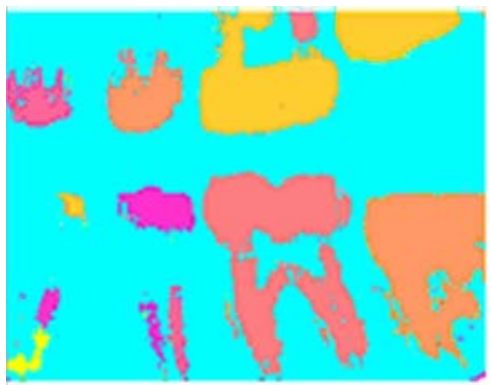

(d)

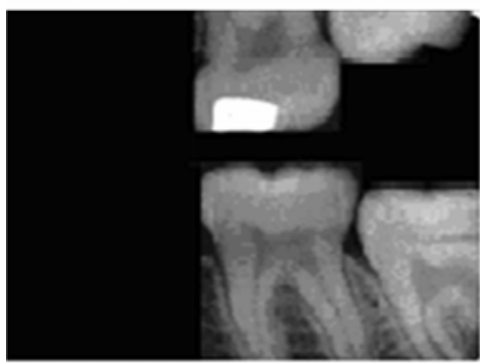

(g)

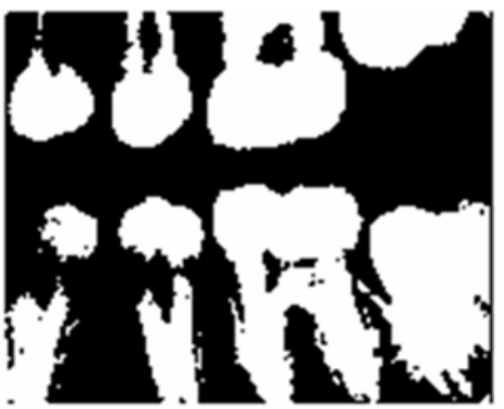

(b)

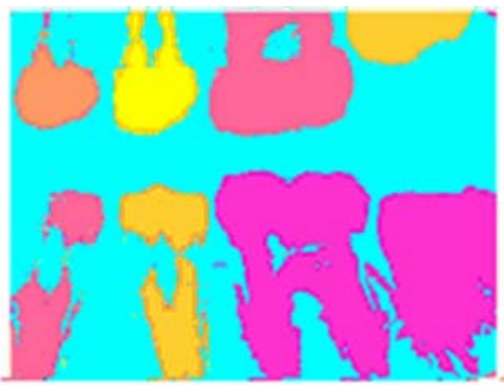

(e)

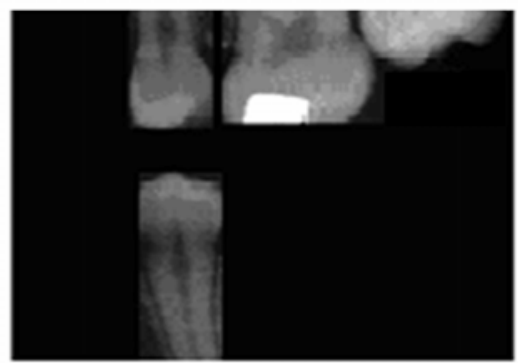

(h)

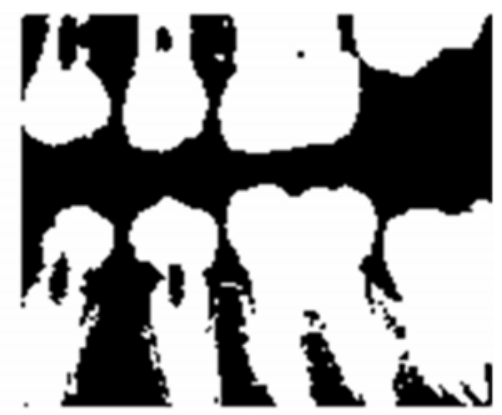

(c)

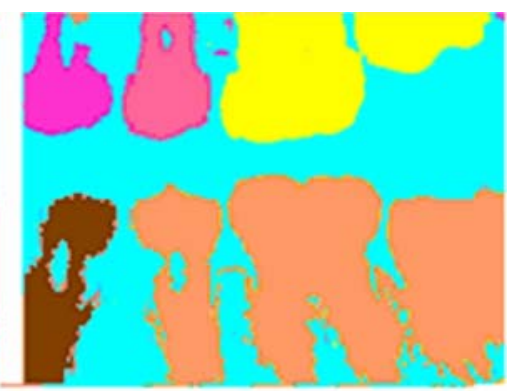

(f)

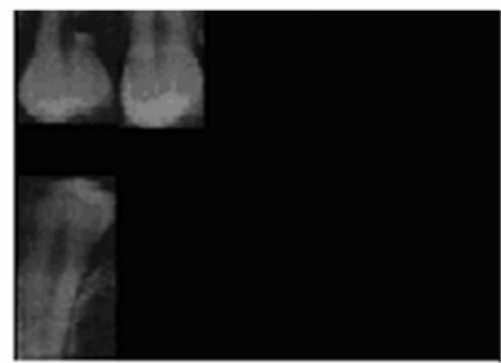

(i)

Figure 20: (a)-(c) Thresholded Images. (e)-(g) Result of connected component labeling for (a)-(c). (g)-(i) Proper ROIs generated from (e)-(g).

Images in Figure 20 (g)-(i) show the proper ROIs. If two or more proper ROIs are generated from the three different thresholds for the same tooth, we unify them to generate the single ROI. The union of proper ROIs represents the final results of segmentation as shown in Figure 21.

Table 2: Rules used in refinement stage to determine the proper ROIs.

\begin{tabular}{cccc}
\hline \hline View Type & ROI height & ROI width & ROI area \\
\hline Bitewing & $0.15 \mathrm{~h}<$ height $<0.7 \mathrm{~h}$ & $0.15 \mathrm{w}<$ width $<0.4 \mathrm{w}$ & Area $>0.0225 \mathrm{hw}$ \\
Periapical & $0.3 \mathrm{~h}<$ height $<0.9 \mathrm{~h}$ & $0.15 \mathrm{w}<$ width $<0.5 \mathrm{w}$ & Area $>0.045 \mathrm{hw}$ \\
\hline
\end{tabular}




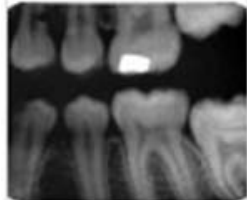

(a)

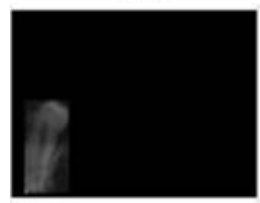

(f)

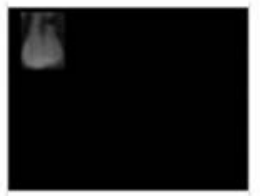

(b)

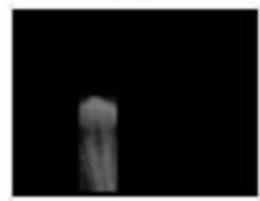

(g)

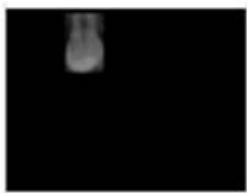

(c)

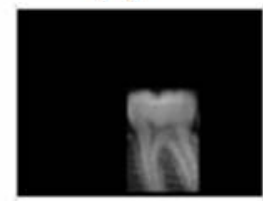

(h)

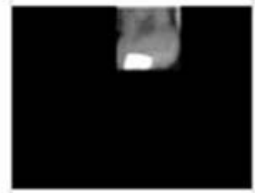

(d)

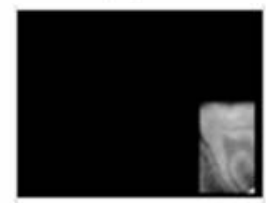

(i)

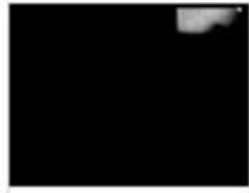

(e)

Figure 21: (a) Original image. (b)-(i) Results of segmentation.

The previous example shows the segmentation stages that applied on bitewing dental images. Figure 22 shows the results of segmentation stages applied on periapical dental images.

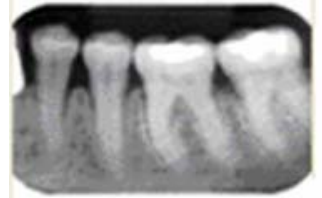

(a)

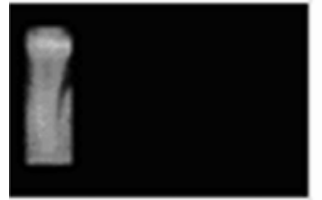

(e)

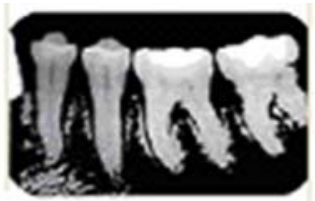

(b)

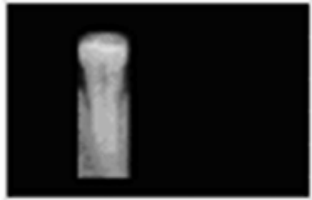

(f)

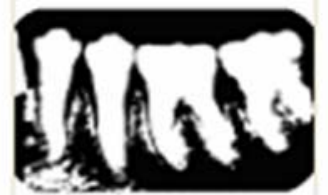

(c)

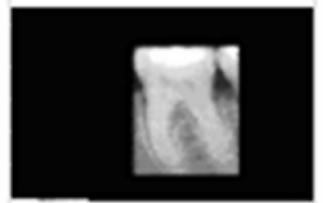

(g)

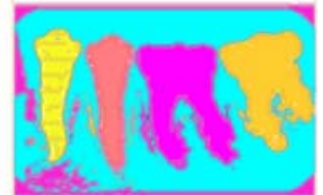

(d)

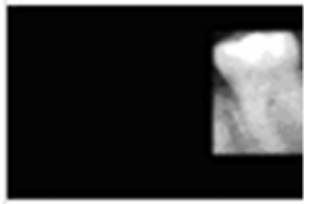

(h)

Figure 22: (a) Original image, (b) Filtered image, (c) Thresholded Images ,(d) Result of connected component labeling, (e)-(h) Proper ROIs.

Figure 23, Figure 24, and Figure 25 show some samples of image segmentation results. The images in Figure 23 have fully succeeded; the images in Figure 24 have partially succeeded, while the images in Figure 25 have failed to give any ROI. In each of Figure 23 and Figure 24 , there are two rows of images, the upper row shows the original images, while the lower row shows teeth obtained from segmentation. It is obvious that the better quality of the dental image the more number of ROIs can be extracted from that image. 

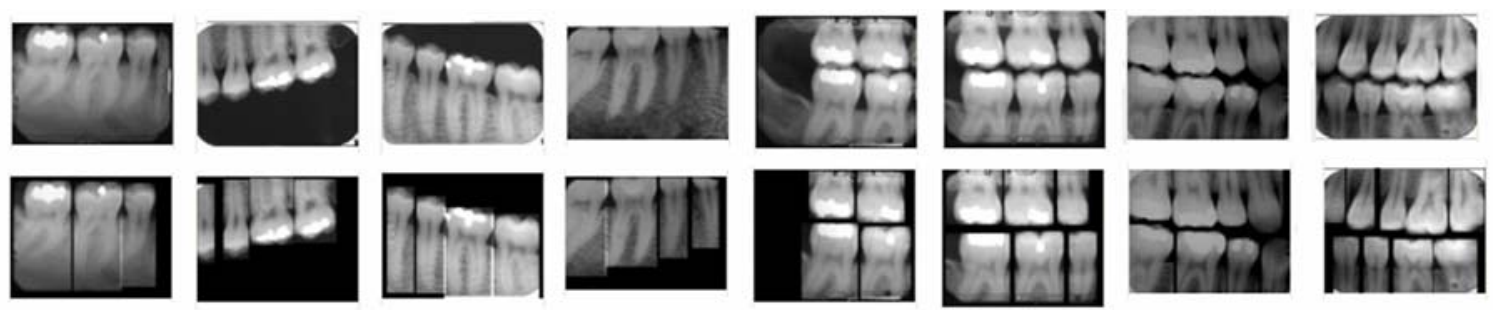

Figure 23: Examples of fully successful images and their segmentation outcomes
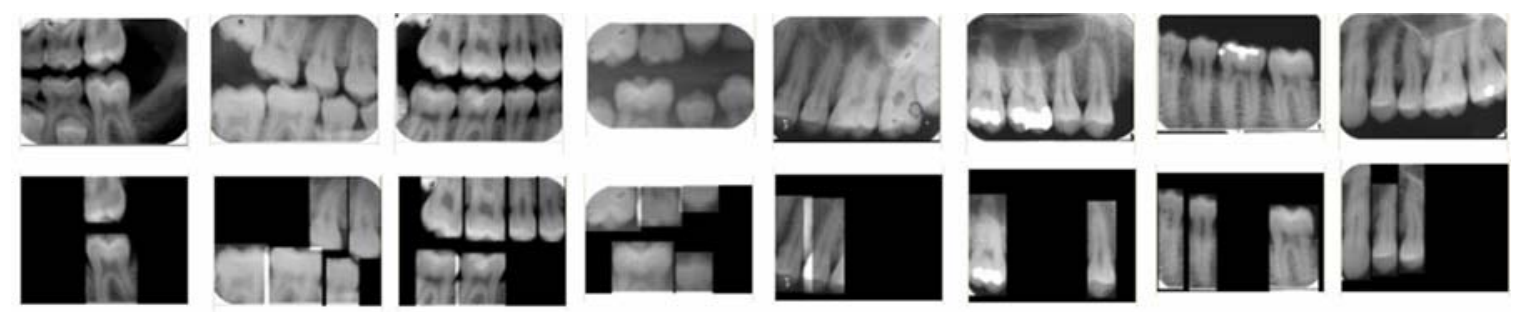

Figure 24: Examples of partially successful images and their segmentation outcomes
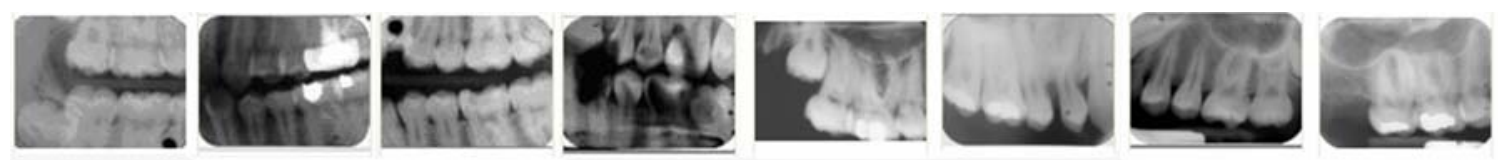

Figure 25: Examples of completely failed images where no tooth can be detected.

\subsection{Performance Assessment in the Dental Image Segmentation}

In this section, we compare the performance of the segmentation algorithm presented in previous section (with and without the enhancement step proposed in [111]). We also compare the performance with those presented in [8][9][10]. We empirically compare between these algorithms on basis of teeth count and their time complexities.

\subsubsection{Teeth Count}

We follow the performance evaluation methodology proposed in [84] in order to compare the performance of the algorithms. Our experiments use two test sets of 500 bitewing and 130 periapical dental radiographic films selected from large dental radiographic databases [115][116]. All films in the bitewing radiographic set contain up to 10 teeth per film, and films in the periapical radiographic set contain up to 5 teeth per film. 
In counting the number of correctly detected teeth in a film, we visually inspect the outcome of segmentation for each film using a simple rule of object containment within each segment of a given film.

Testing results of the algorithm we propose in the previous section for bitewing radiographic set before and after enhancement are shown in Figure 26 and Figure 27 respectively, while testing results for periapical radiographic set before and after enhancement are shown in Figure 28 and Figure 29 respectively.

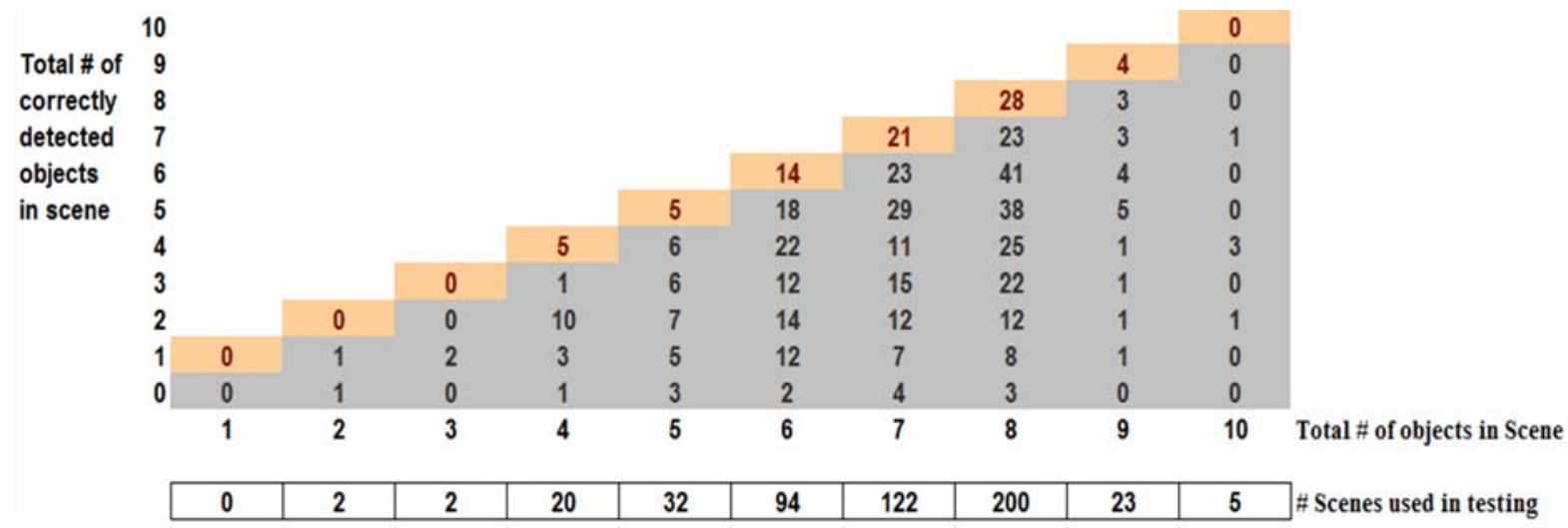

Figure 26: Testing results for of our teeth segmentation algorithm with bitewing views.

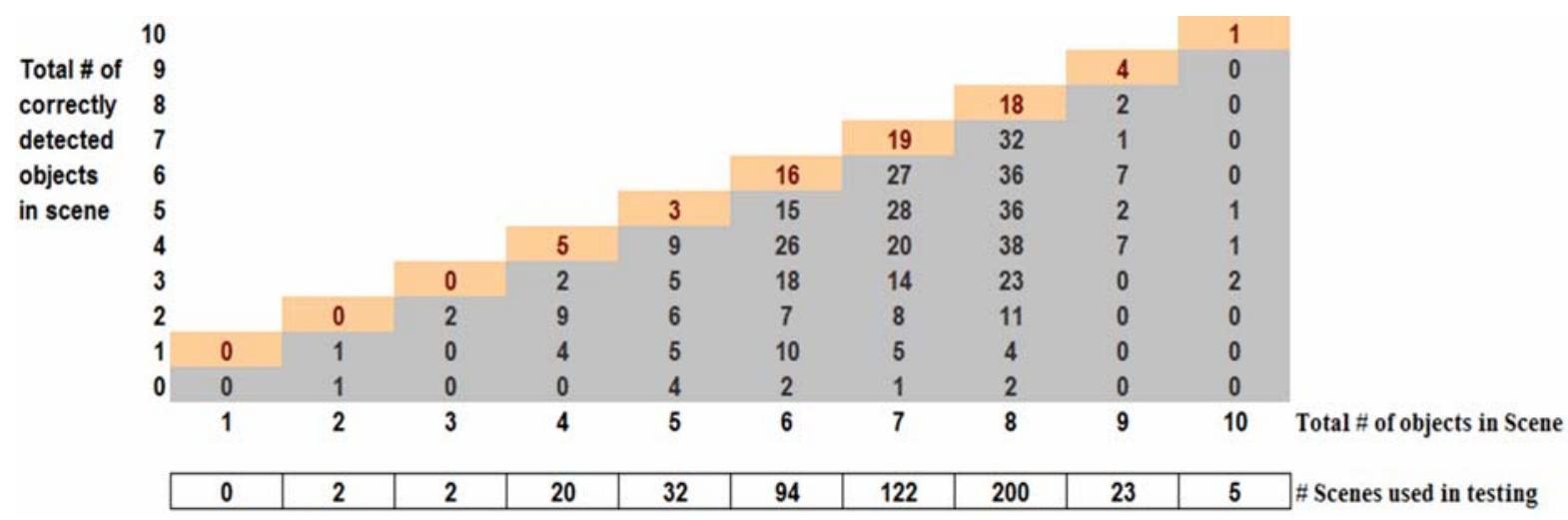

Figure 27: Testing results for of our teeth segmentation algorithm with contrast stretched bitewing views. 


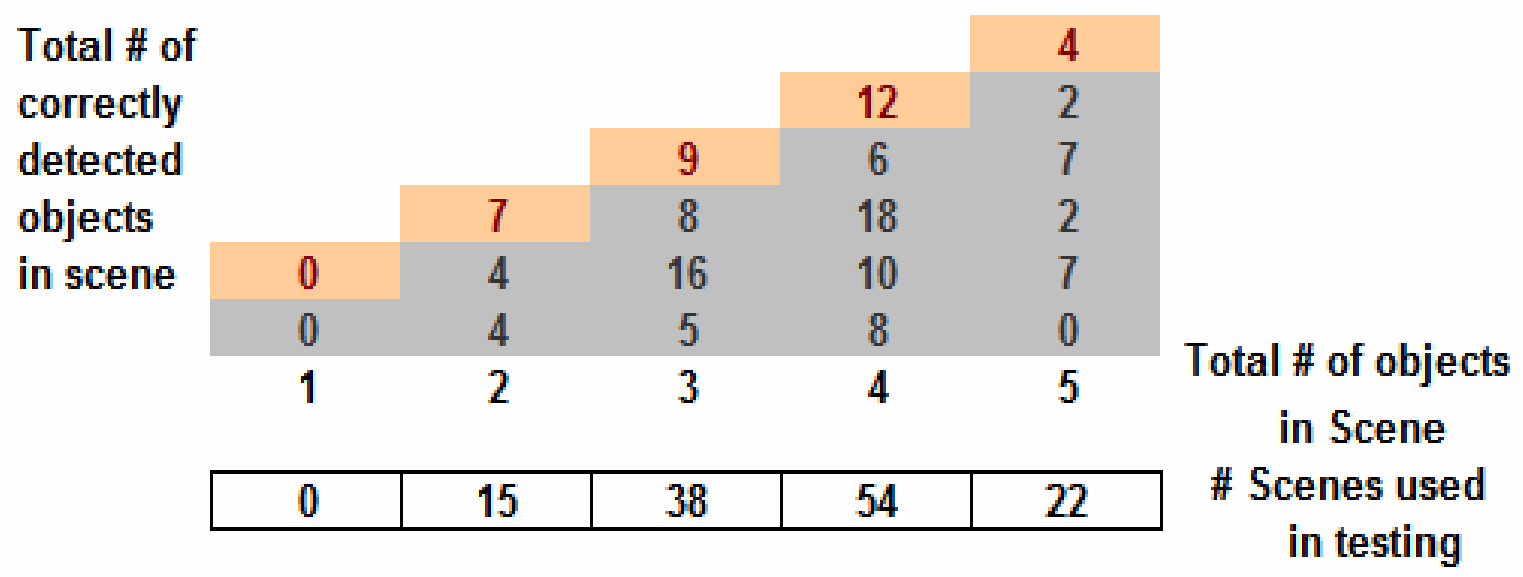

Figure 28: Testing results for of our teeth segmentation algorithm with periapical views.

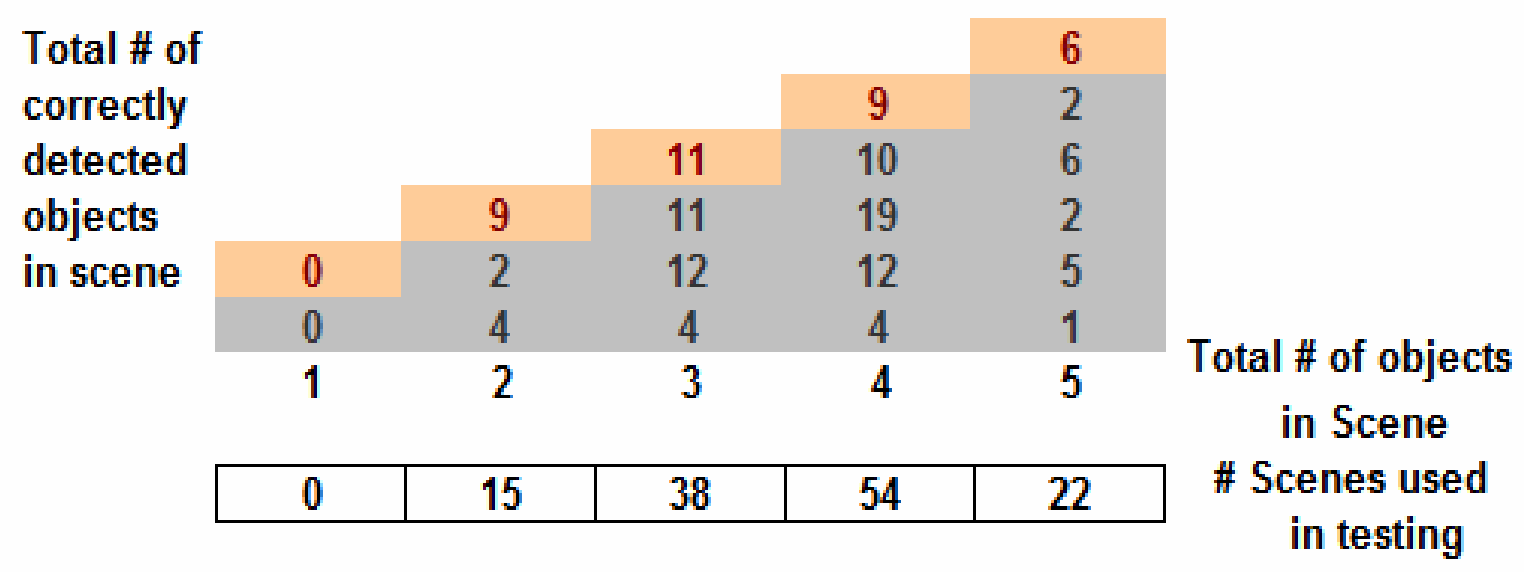

Figure 29: Testing results for of our teeth segmentation algorithm with contrast stretched periapical views.

Testing results of the algorithms proposed in [8][9], and [10] are shown in Figure 30, Figure 31, and Figure 32 respectively. The bitewing set of dental images used for testing is the same as the one used for testing our segmentation approach. Figure 33 shows a graphical comparison using the metrics in [84] between the proposed dental film segmentation algorithm, the proposed algorithm after enhancement presented [111], and the analogous algorithms proposed in [8] [9][10]. 


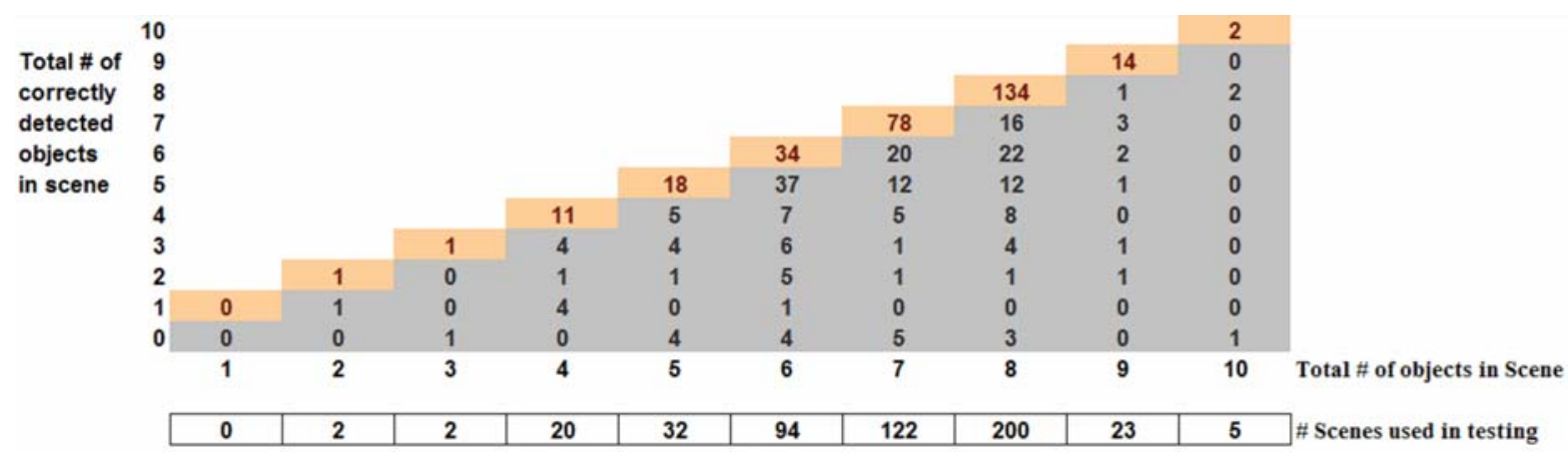

Figure 30: Testing results of Jain and Chen segmentation algorithm [8].

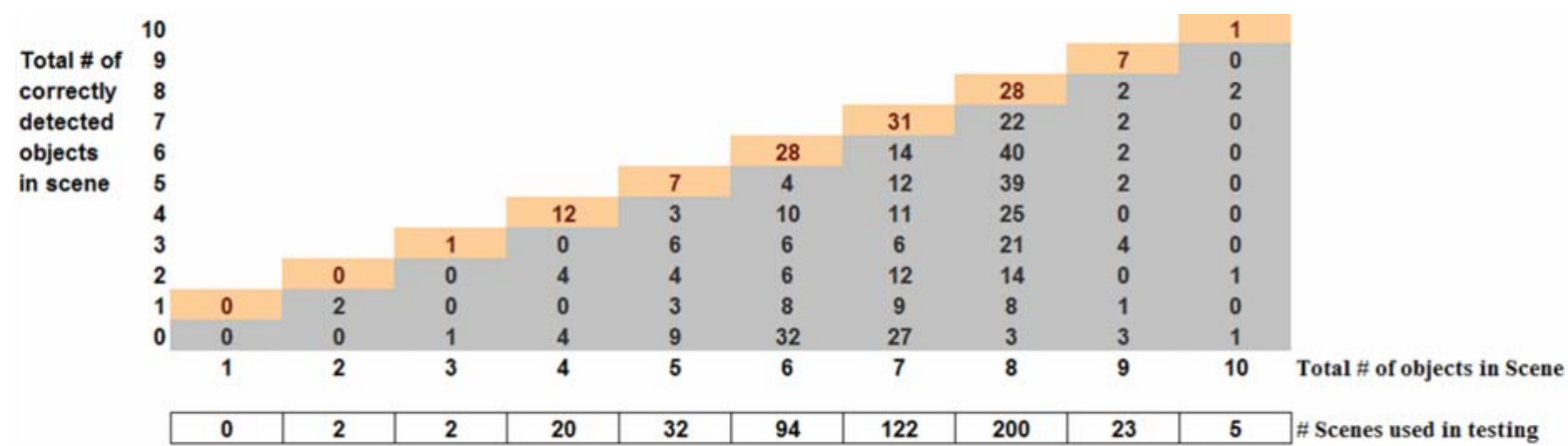

Figure 31: Testing results of Nomair and Abdel-Mottaleb segmentation algorithm [9].

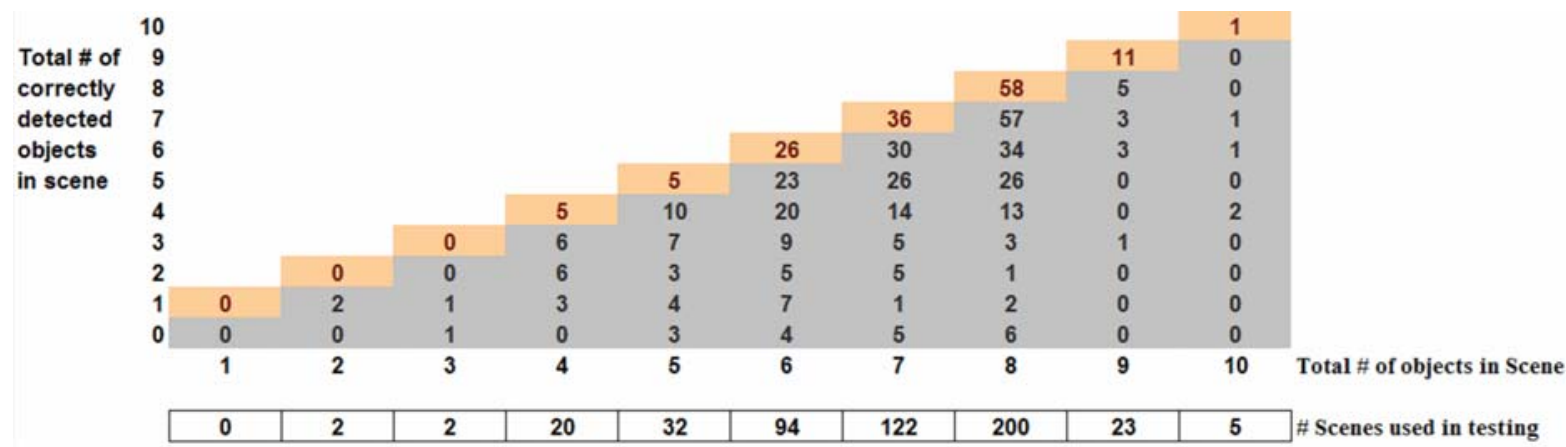

Figure 32: Testing results of Zhou and Abdel-Mottaleb segmentation algorithm [10]. 


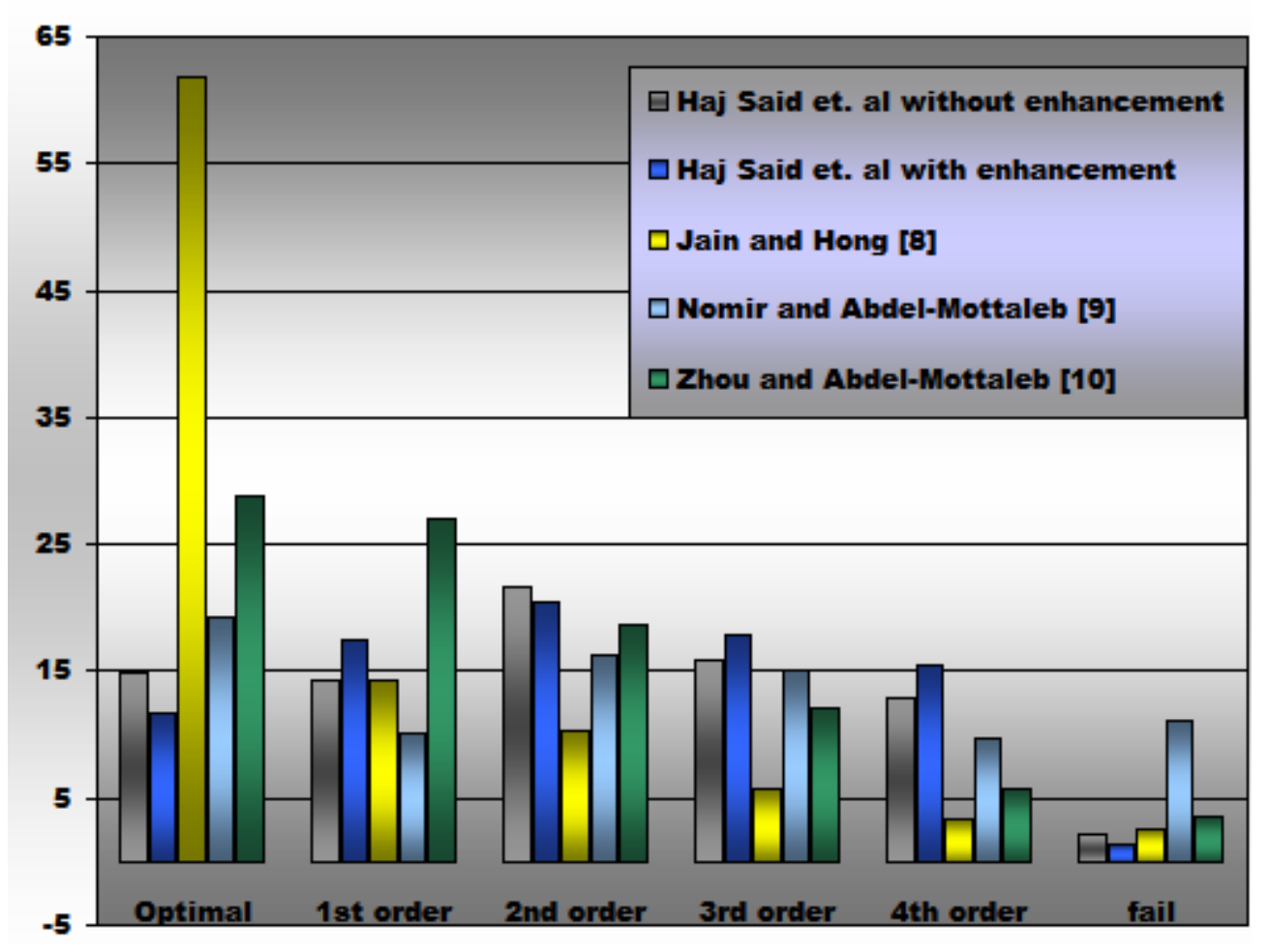

Figure 33: Performance comparison between bitewing segmentation algorithms.

While optimality and failure percentages capture instances of extreme performance of a segmentation algorithm, sub-optimality measures capture the performance of algorithms in between the two extremes. For example, in teeth segmentation, a 1st. order sub-optimality is a measure of the tendency of the algorithm to miss detection of exactly one tooth and detect all the others, but with no failure.

In practical cases, it is difficult to achieve optimal performance with $100 \%$ of images, and when comparing segmentation algorithms one should favor those whose failure rates are the lowest and their optimality and low-order measures of sub-optimality predominate the testing results [84].

The failure rate is especially important, when assessing teeth segmentation algorithms, since those films where no teeth can be properly segmented cannot be used in the identification process.

We conclude the following observations from Figure 33 and Table 3: 
- The optimality of the algorithm proposed in [8] is superior to other algorithms, but it is still a semi-automated algorithm.

- The optimality of the algorithm proposed in [10] is superior to other full-automated algorithms.

- Failure rate of the proposed algorithm is the lowest (see Table 3).

- Slight improvement has been made in the failure rate of the proposed algorithm after applying the image enhancement scheme.

- Enhancement dropped the optimality of the proposed algorithm by approximately $3 \%$ for the cases lie between the axis of optimality and the second order sub optimality.

- Enhancement increased the percentage of the cases lie between optimality and 4th order sub-optimality to $83 \%$.

Table 3: Comparison between the failure rates of the variant teeth segmentation algorithms.

\begin{tabular}{cccccc}
\hline \hline Algorithm & $\begin{array}{c}\text { Haj Said, } \\
\text { Nassar, Fahmy, } \\
\text { and Ammar } \\
\text { algorithm without } \\
\text { enhancement }\end{array}$ & $\begin{array}{c}\text { Haj Said, } \\
\text { Nassar, Fahmy, } \\
\text { and Ammar } \\
\text { algorithm with } \\
\text { enhancement }\end{array}$ & $\begin{array}{c}\text { Jain } \\
\text { and } \\
\text { Hong } \\
\text { [ 8 ] }\end{array}$ & $\begin{array}{c}\text { Nomair and } \\
\text { Abdel-Mottaleb } \\
\text { [ } \text { ] ] }\end{array}$ & $\begin{array}{c}\text { Zhou and } \\
\text { Abdel- } \\
\text { Mottaleb } \\
{[10]}\end{array}$ \\
\hline Failure rate & $2.12 \%$ & $1.27 \%$ & $2.61 \%$ & $11.18 \%$ & $3.47 \%$ \\
\hline
\end{tabular}

While the algorithms proposed in [8], [9][10] do not work with the periapical dental radiographs, Figure 34 shows a graphical comparison using the metrics in [84] between the proposed algorithm and the proposed algorithm with enhancement. It is clear that the enhancement decreases the failure rate from $9.4 \%$ to $6.7 \%$, and it improves the optimality and $1^{\text {st }}$ order sub optimality. 


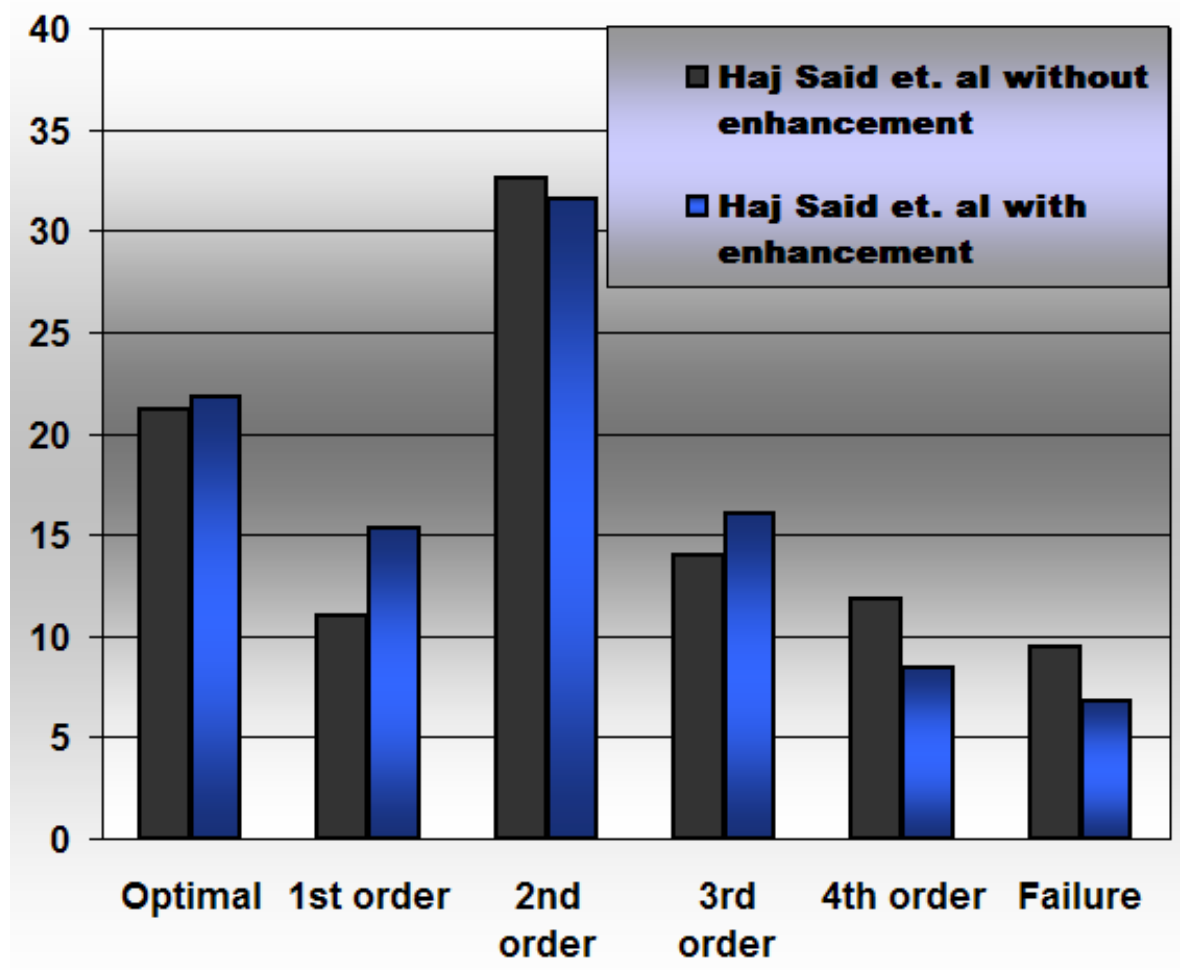

Figure 34: Performance comparison between proposed algorithm and proposed algorithm with enhancement for periapical views.

\subsubsection{Time Complexity}

To compare between the time complexities of the proposed algorithm and those proposed in [8][9], and [10], we used 40 bitewing films with different dimensions. We invoked

MATLAB ${ }^{\circledR}$ implementations of each of the algorithms on an Intel Pentium 4® $2.00 \mathrm{GHZ}$, 512 MB DRAM platform. Table 4 summarizes the outcome of the time complexity comparison between the four teeth segmentation algorithms; $h$ is the image height, $w$ is the image width, and $n$ is the size of window used in adaptive thresholding.

Figure 35 records the execution times of the four teeth segmentation algorithms for each of the 40 bitewing films. Based on Figure 35 and Table 4, we observe that: (i) the proposed algorithm is the fastest among the other algorithms, (ii) the difference in the average time execution between the proposed algorithm, algorithm [8], and algorithm [10] is small, (iii) the time complexity of the algorithm [9] is significant comparing to the other algorithms. 
Table 4: Comparison between the time complexities of the four teeth segmentation algorithms.

\begin{tabular}{|c|c|c|c|c|}
\hline Algorithm & $\begin{array}{c}\text { Haj Said, } \\
\text { Nassar, Fahmy, } \\
\text { and Ammar } \\
\text { Algorithm }\end{array}$ & $\begin{array}{l}\text { Jain and } \\
\text { Hong } \\
\text { [ 8 ] }\end{array}$ & $\begin{array}{c}\text { Nomair } \\
\text { and Abdel- } \\
\text { Mottaleb [ } 9 \text { ] }\end{array}$ & $\begin{array}{c}\text { Zhou and } \\
\text { Abdel-Mottaleb } \\
{[10]}\end{array}$ \\
\hline $\begin{array}{l}\text { Average time } \\
\text { complexity } \\
\text { (sec.) }\end{array}$ & 4.614 & 5.658 & 57.251 & 7.323 \\
\hline $\begin{array}{c}\text { Time } \\
\text { complexity } \\
\text { order }\end{array}$ & $\mathrm{O}(\mathrm{hw})$ & $\mathrm{O}(\mathrm{hw})$ & $\mathrm{O}(\mathrm{nhw})$ & $\mathrm{O}(\mathrm{nhw})$ \\
\hline
\end{tabular}

\subsection{Ear Segmentation from Facial Images}

In order to segment the ear in the facial images, we need first to extract the face from the background, and then segment the ear from the detected face. We define two main classes of objects in the facial images; face that map to areas with variant grayscale, and background that maps area with smooth grayscale.

\subsubsection{Face Detection}

While we are working with colored RGB facial images, we consider each of the color components of the image as a single grayscale image. We apply the morphology operations on each of color components of the RGB image. Figure 36 shows the main steps of face extraction from the background. We first subtract the subject image from its erosion, and then we threshold the resulted image using basic thresholding (255/10). We use a disk structuring element with dimension [w/40] for erosion operation, where $\mathrm{w}$ is the width of the image. Finally we apply binary dilation and holes filling to ensure that the whole face is comprised.

Figure 37 shows the face detection steps in details that corresponded to steps shown in Figure 36. Figure 38 shows the face boundary as a result of this stage. 


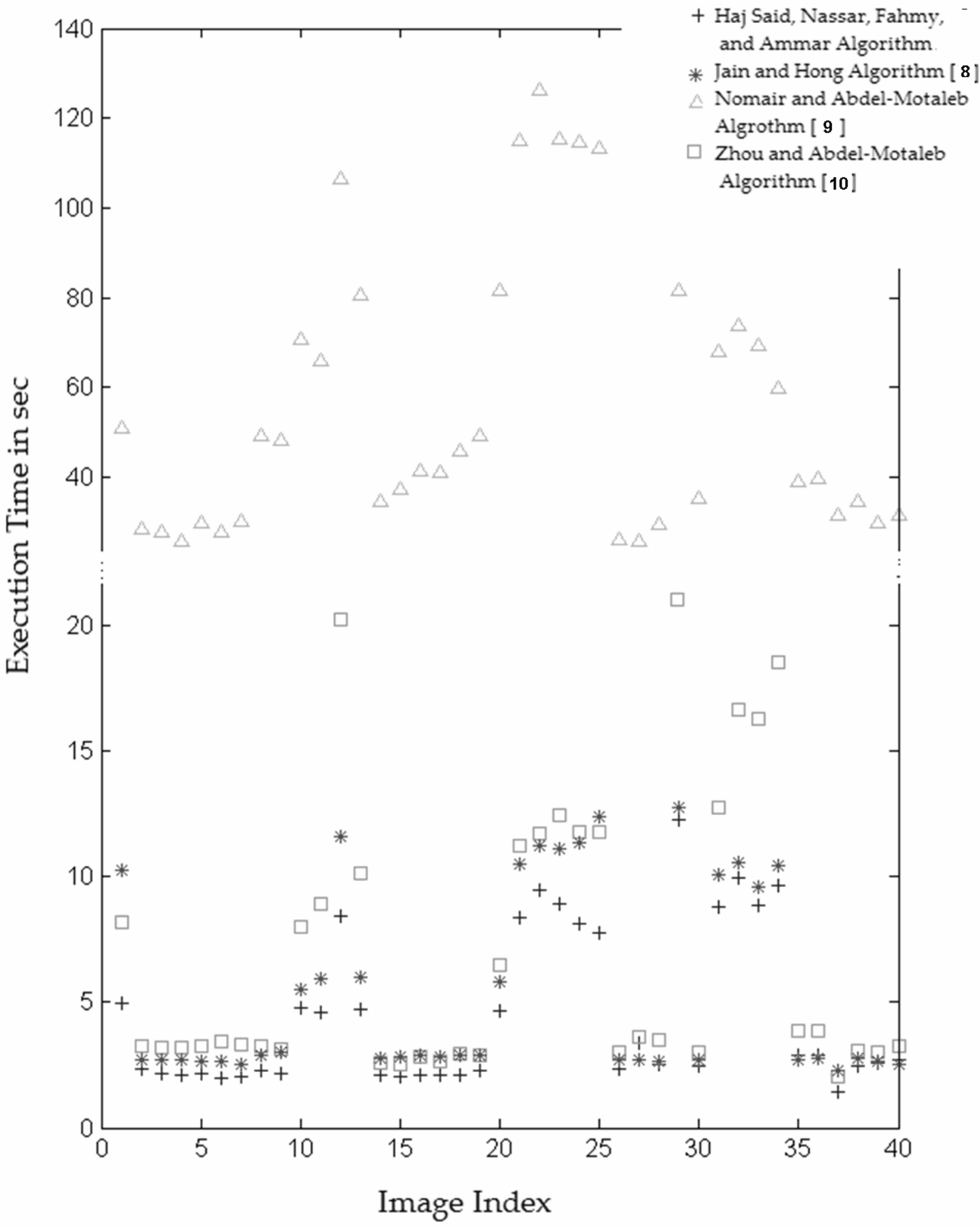

Figure 35: Time complexity distribution of 40 images for variants algorithms 


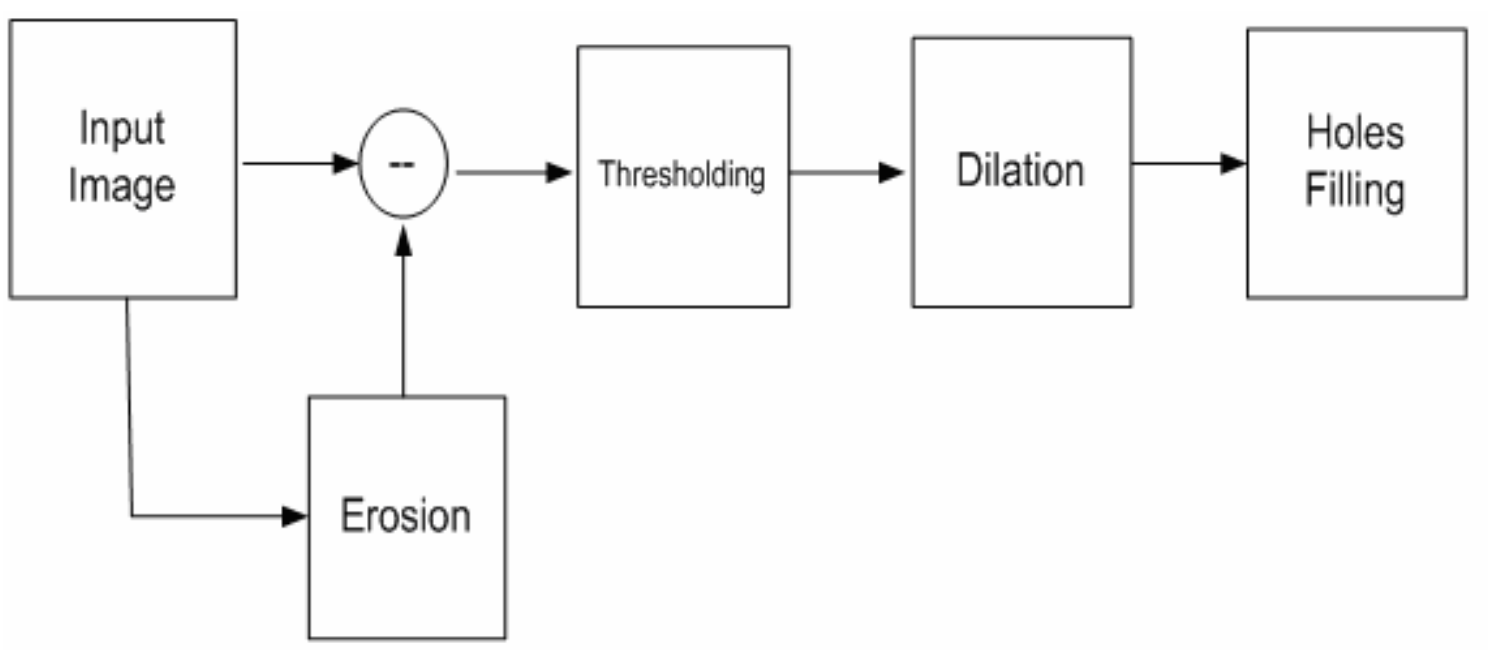

Figure 36: Main Stages of Face Detection

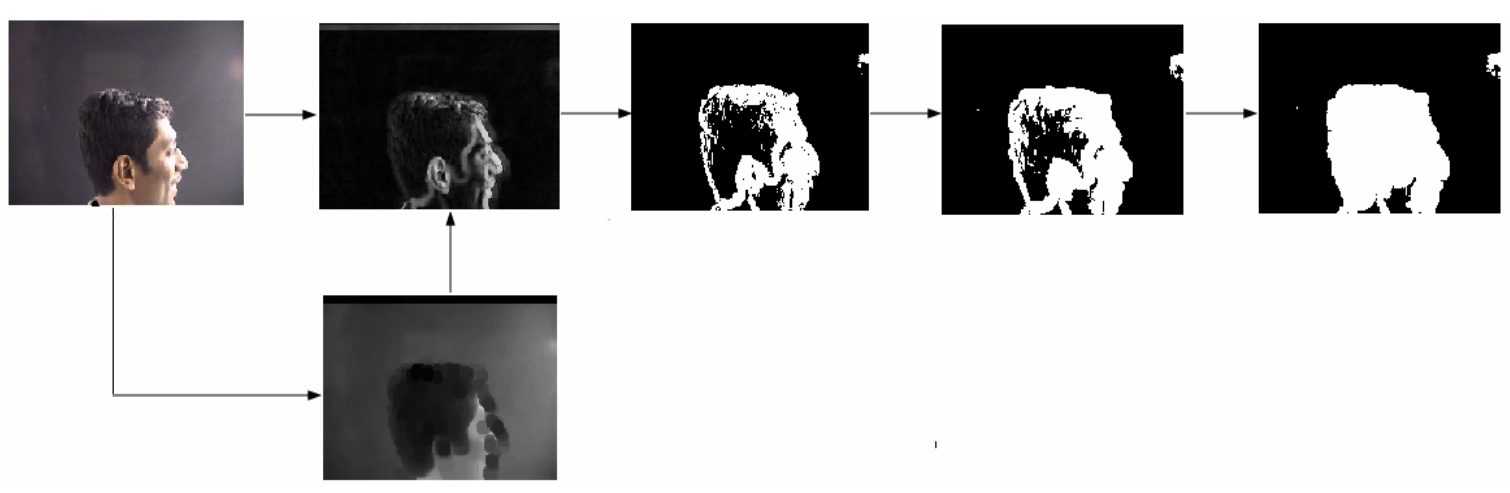

Figure 37: An Example shows the stages of face detection

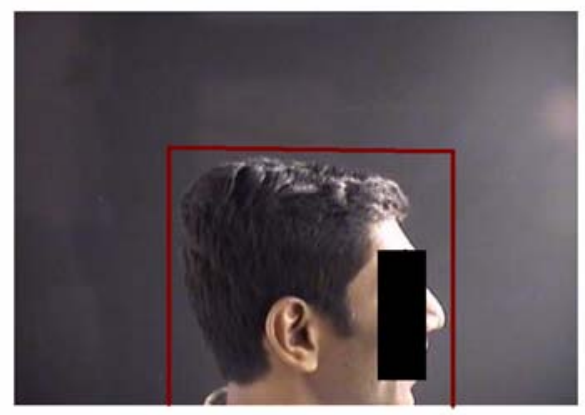

Figure 38: Face Boundary

\subsubsection{Filtering Stage in the Facial images}


The main goal of filtering is to emphasize the bright and variant grayscale areas and to suppress the dark and smooth grayscale areas. We apply opening top hat transformation, which is defined by subtracting morphological opening of the image from itself. Opening top hat transformation provides an excellent tool for detecting pixels that are bright on the surrounding dark areas, and it locally performs suppression of dark and smooth areas. The idea of using top hat transformation in order to emphasize the ear is that i) the ear is surrounded by darker pixels such as hair and smooth areas such as cheek, ii) the ear object itself contains dark gaps which lead to emphasize the ear edges after applying the transformation.We use a disk structuring element with dimensions [w/30] for facial images and $(255 / 20)$ for basic thresholding. Figure 39 shows the facial image before and after filtering. It is clear how the ear and some part of the face have been emphasized while the other objects that are dark and smooth have been suppressed.
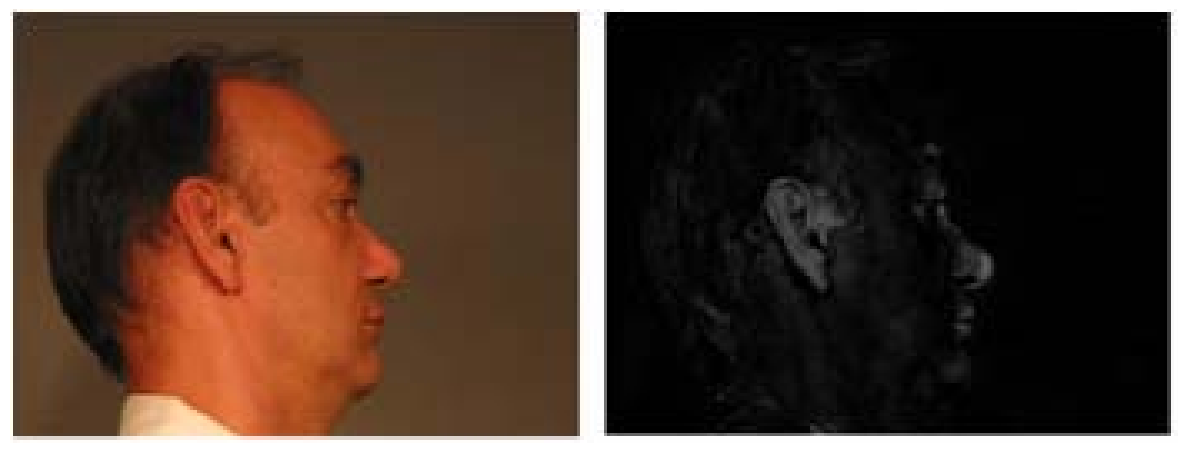

Figure 39: Original and filtered images.

\subsubsection{Thresholding in the Facial Images}

We use the K-mean Clustering algorithm in order to produce the binary image from the filtered imaged that produced from the previous stage. We first partition the image pixels into two sets based on their intensity; one belongs to low intensity set, and the other to the high intensity one. After convergence, we assign the highest intensity value in the low intensity set as the threshold value. Figure $\mathbf{4 0}$ shows thresholded image that produced from partitioning the filtered image into two clusters using K-mean clustering algorithm. 


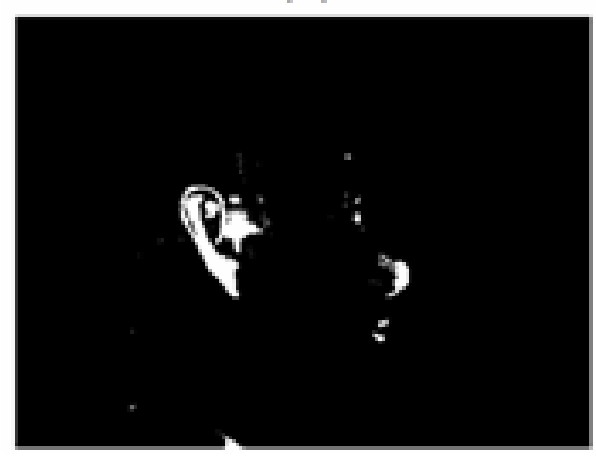

Figure 40: Thresholded image

\subsubsection{Connected component Labeling in Facial images}

Using the thresholded image obtained from the previous stage, we determine the number of potential regions in the facial image by running connected component labeling algorithm, We used an 8-connected neighborhood to group the pixels and to label them. The idea behind choosing 8-connected neighborhood instead of 4-connected neighborhood is to produce less and larger regions. The connected components can be corresponded to an ear or any other part of the face as shown in Figure 41.

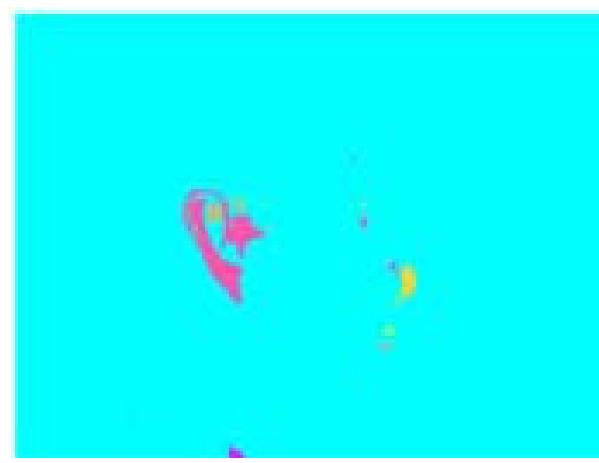

Figure 41: Result of connected component labeling of the thresholded image

\subsubsection{Refinement in Facial Images}

The purpose of refinement stage is i) to remove the smaller connected components obtained from the previous stage whose areas are less or greater than threshold value, and ii) to analyze the remaining connected components based on their geometric properties including position and dimensions. We used the following rules to determine the proper ROIs and to eliminate the improper ROIs: 
1. The area of object should be less than $10 \%$ of the area of the detected face, and greater than $2.5 \%$ of the area of the detected face.

2. The object's centroid locates in the middle quarters of the detected face .

1. The height of the object is less than $0.4 h_{f}$ and greater than $0.15 h_{f}$, where $h_{f}$ is the height of detected face

2. The width of the object is less than $0.4 w_{f}$ and greater than $0.1 w_{f}$, where $w_{f}$ is the width of the detected face

3. The width of the object should not increase its height.

4. The centriod of object should not belong to the brighter half of the detected face.

Figure 42 shows the result of the refinement stage where the ear is bounded by red rectangular.

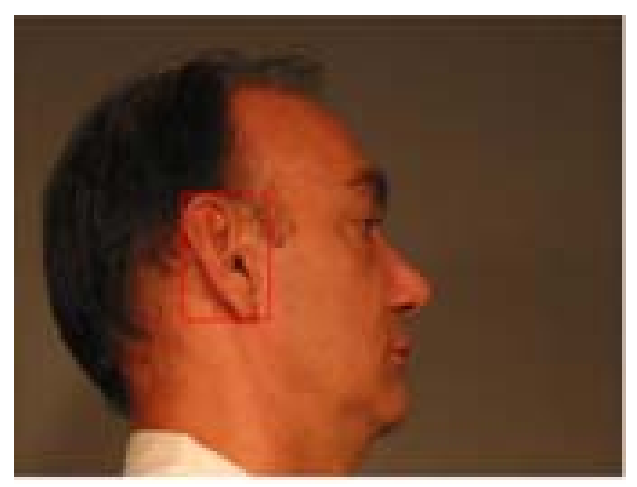

Figure 42: Detected ear after refinement stage.

Figure 43, Figure 44, Figure 45, and Figure 46 show some samples of ear segmentation results from facial images [112][113], and [114]. All images have been chosen from different sets of facial images with different background color and different views. The images in Figure 43 and Figure 45 have fully succeeded; the images in Figure 44 and Figure 46 have partially succeeded or completely failed to give an object. 

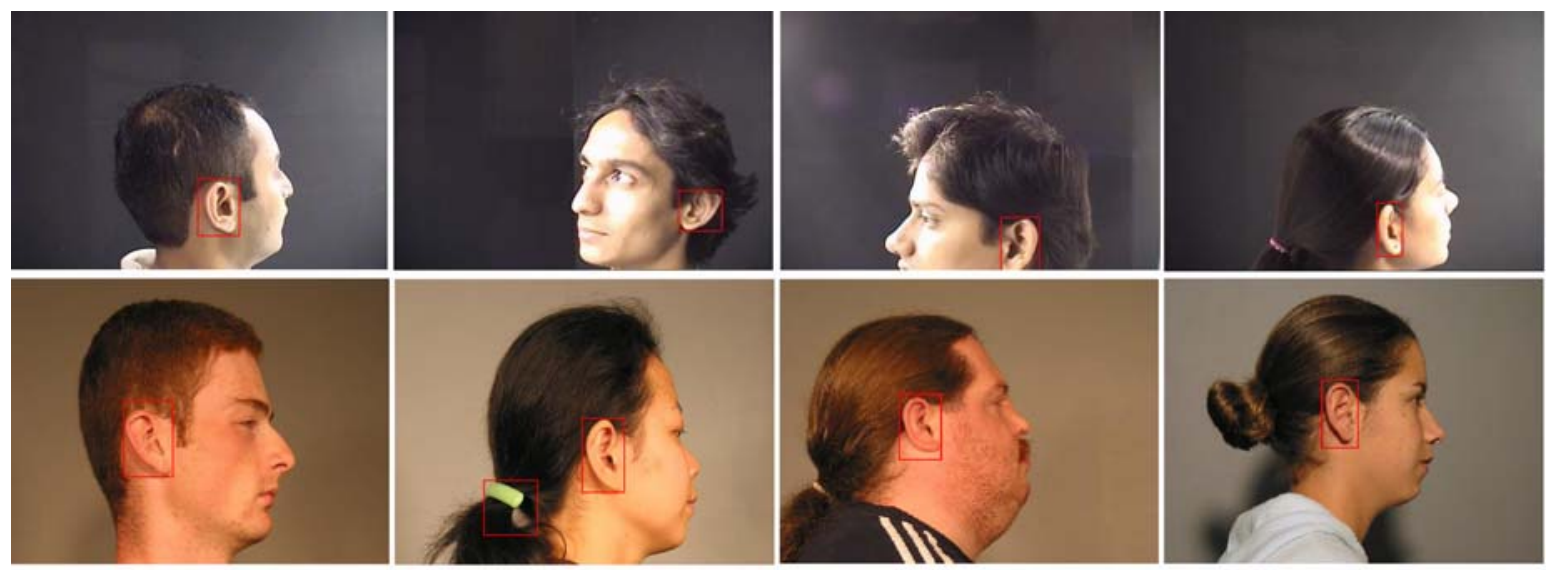

Figure 43: Examples of completely successful images and their segmentation outcomes chosen from [112][113].

The results show that the segmentation algorithm effectively works i) with different views and angles (-10 to 75 degree for each side), ii)with different genders, iii) with different races, iv) and with presence of objects interfering such as bears, hair, earrings, glasses and hats. In some cases, the effect of illumination variation, hair, or earrings will lead to partially succeeded or totally failed to detect the ear. However; there no subject failed to give any correct detected ear from his/her video frames.
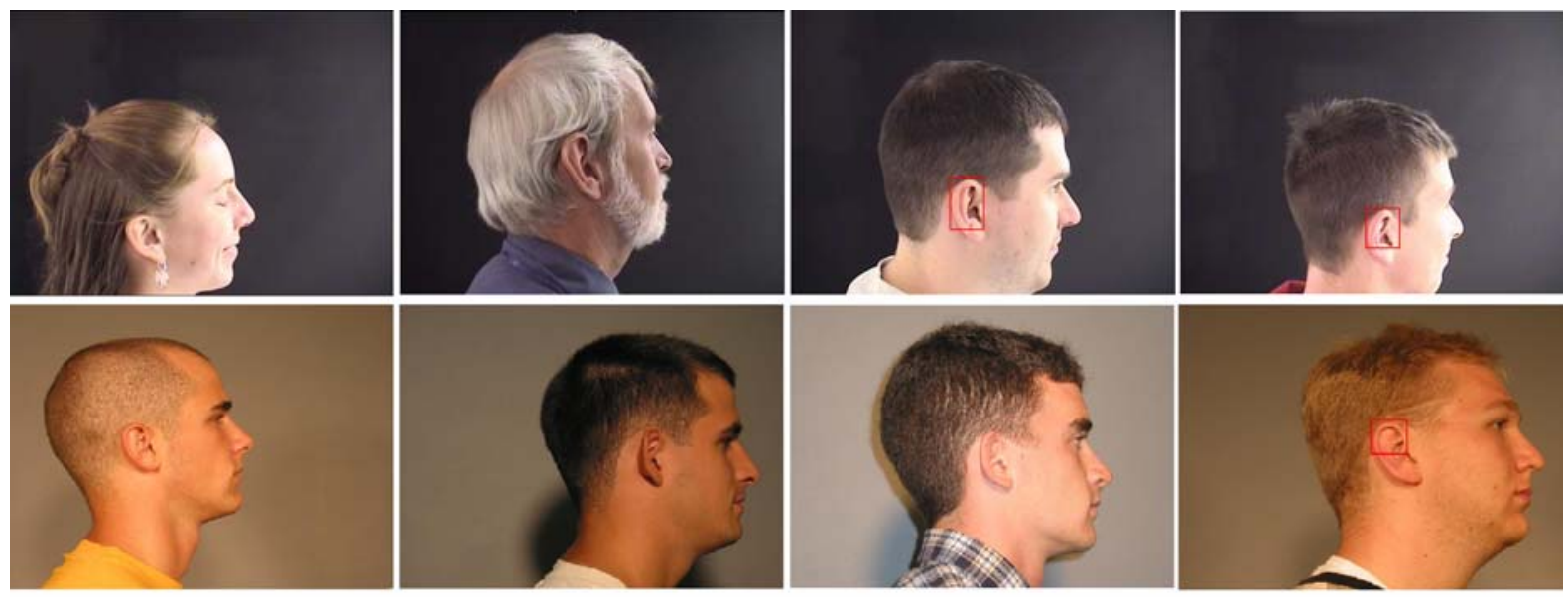

Figure 44: Examples of completely failed images and partially successful succeeded images and their segmentation outcomes chosen from [112][113]. 


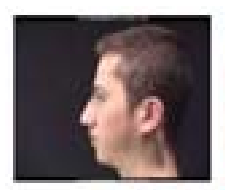

a

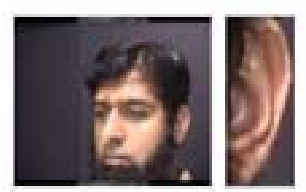

e

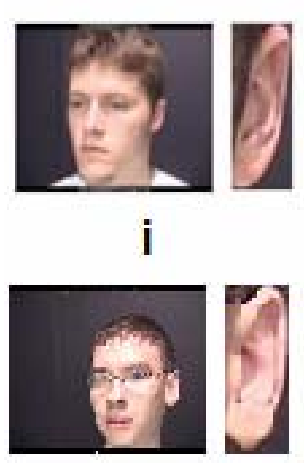

m

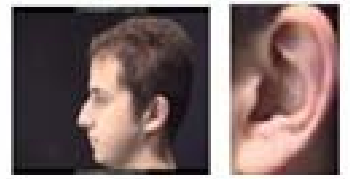

b

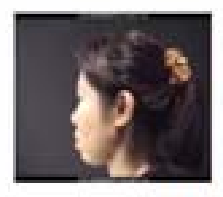

f
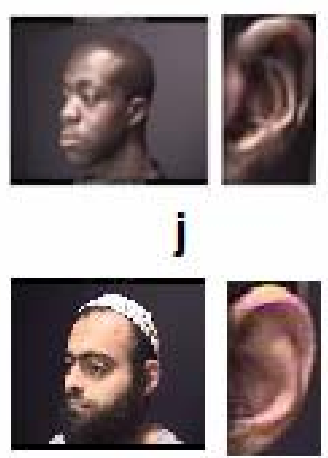

n

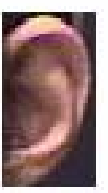

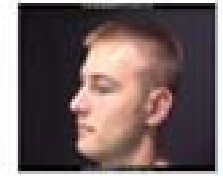

C

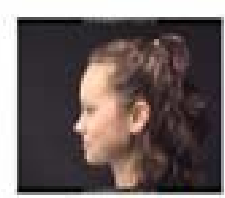

g

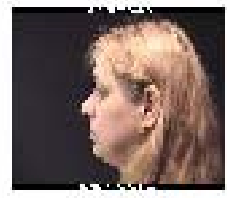

k

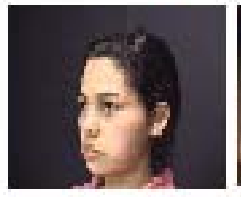

0

.
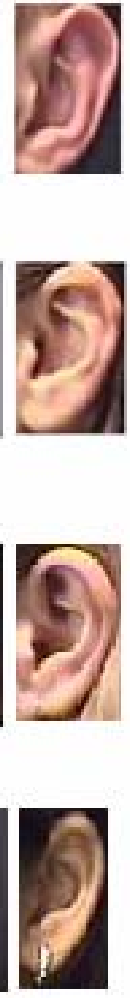
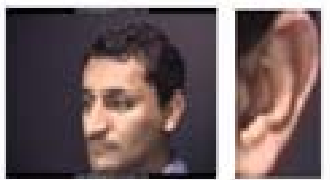

d

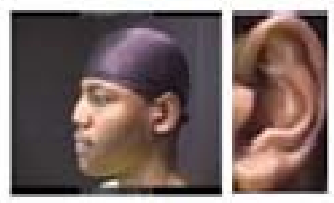

h

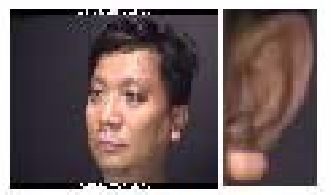

I

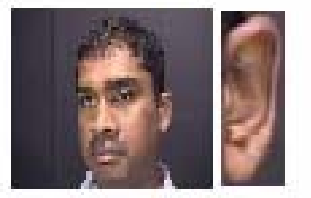

p

Figure 45: Examples of completely successful images and their segmentation outcomes.

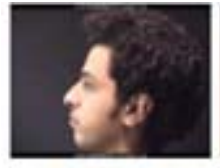

a

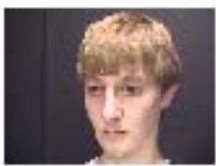

d
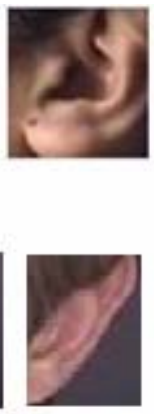

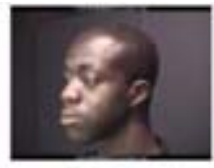

b

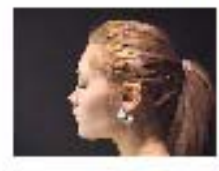

e
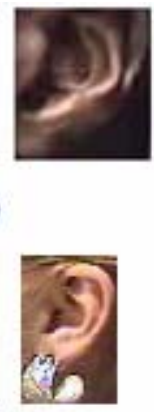

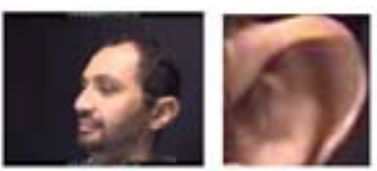

C
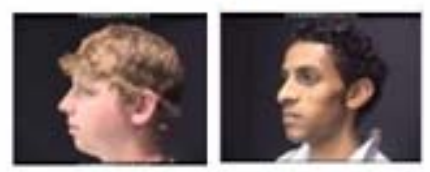

f

Figure 46: Examples of completely failed and partially succeeded images and their segmentation outcomes.

\subsection{Performance Assessment of Ear Segmentation}

In order to evaluate the performance of the ear segmentation, we apply it on three different test sets of facial images taken under different conditions and different quality. The testing sets are: 
1- The first set contains 150 images [113] taken from 50 video frames for 50 subjects with different views and variant heights.

2- The second set contains 100 profile images [112] with different background and variant illumination conditions.

3- The third set contain 3500 images taken from 226 video frames [114] for 226 subjects with different views.

After that, we compare the performance of the segmentation algorithm with the one presented in [11] on basis of their output and their time complexities. All the images used for testing have size of $640 \times 480$. We visually inspect the outcome of segmentation for each image using a simple rule of object containment within each segment of a given image. We invoked MATLAB ${ }^{\circledR}$ implementations of each of the algorithms on an Intel Pentium M 1.70 GHZ, 1024 MB SDRAM platform.

Testing results of the proposed algorithm and the one proposed in [11] for the three test sets are shown in Table 5, Table 6, and Table 7. The tables show the percentage of fully succeeded images, partially succeeded images, and failed images. Tables also show the outcome of the time complexity comparison between the two algorithms for the three test sets.

The results show that our algorithm is superior to the algorithm proposed in [11] in terms of performance. In addition, our approach works with different views, angles, height, background, genders, races, and it worked properly with three different databases. The percentage of the success exceeds $90 \%$ of the images, and no subject has failed to give any successful segments.

Table 5: Comparison results between our approach and the approach presented in [11] for the first testing set [113]

\begin{tabular}{|c|c|c|c|c|}
\hline Approach & $\begin{array}{c}\text { Percentage of fully } \\
\text { succeeded images }\end{array}$ & $\begin{array}{c}\text { Percentage of } \\
\text { partially succeeded } \\
\text { images }\end{array}$ & $\begin{array}{c}\text { Percentage of fully } \\
\text { failed images }\end{array}$ & $\begin{array}{c}\text { Time } \\
\text { complexity }\end{array}$ \\
\hline $\begin{array}{c}\text { Our } \\
\text { Approach }\end{array}$ & $92 \%$ & $4.66 \%$ & $3.3 \%$ & 5.21 second \\
\hline $\begin{array}{c}\text { Approach } \\
\text { presented in [1] }\end{array}$ & $29.3 \%$ & $23.3 \%$ & $47.3 \%$ & 2.53 second \\
\hline
\end{tabular}


Table 6: Comparison results between our approach and the approach presented in [11] for the second testing set [112]

\begin{tabular}{|c|c|c|c|c|}
\hline Approach & $\begin{array}{c}\text { Percentage of fully } \\
\text { succeeded images }\end{array}$ & $\begin{array}{c}\text { Percentage of } \\
\text { partially } \\
\text { succeeded images }\end{array}$ & $\begin{array}{c}\text { Percentage of fully } \\
\text { failed images }\end{array}$ & Time complexity \\
\hline $\begin{array}{c}\text { Our } \\
\text { Approach }\end{array}$ & $89 \%$ & $6 \%$ & $5 \%$ & 4.93 second \\
\hline $\begin{array}{c}\text { Approach presented } \\
\text { in [11] }\end{array}$ & $26 \%$ & $30 \%$ & $44 \%$ & 3.72 second \\
\hline
\end{tabular}

Table 7: Comparison results between our approach and the approach presented in [11] for the third testing set [114].

\begin{tabular}{|c|c|c|c|c|}
\hline Approach & $\begin{array}{c}\text { Percentage of fully } \\
\text { succeeded images }\end{array}$ & $\begin{array}{c}\text { Percentage of } \\
\text { partially } \\
\text { succeeded images }\end{array}$ & $\begin{array}{c}\text { Percentage of fully } \\
\text { failed images }\end{array}$ & Time complexity \\
\hline $\begin{array}{c}\text { Our } \\
\text { Approach }\end{array}$ & $92.51 \%$ & $5.17 \%$ & $2.32 \%$ & 5.87 second \\
\hline $\begin{array}{c}\text { Approach presented } \\
\text { in [11] }\end{array}$ & $23.14 \%$ & $21.6 \%$ & $55.26 \%$ & 2.73 second \\
\hline
\end{tabular}

\subsection{Advanced Approach for Dental Image Segmentation}

In order to improve the segmentation performance, we develop different segmentation approach that is based on mathematical morphology and convolution. In this approach, we first blur the image by convolving it using 2D filter Point spread Function PSF that simulates a motion blur and specifies the length and angle of the blur. The blurring process can be defined by:

$$
b(x, y)=f(x, y) * h(x, y)
$$

Where: $\mathrm{b}(\mathrm{x}, \mathrm{y})$ is the blurred image, $\mathrm{f}(\mathrm{x}, \mathrm{y})$ is the original image, and $\mathrm{h}(\mathrm{x}, \mathrm{y})$ is the PSF that is defined by:

$$
\mathrm{h}(\mathrm{x}, \mathrm{y})=\frac{1}{\underline{L}} \delta(\underline{L})
$$

Where $\underline{L}$ is the line segment of length L oriented at an angle of $\theta$ degrees from the x-axis. 
After blurring the image, we subtract it from its original image and then apply basic threshold to get thresholded image. Each pixel has an intensity zero is set to zero, and one otherwise. Figure 47 shows the blurred image and the image resulted from subtracting it from the original image. Figure 48 shows the thresholded image and its complement after filtering the small region of the thresholded image. It is clear that the effect of this process in reducing or eliminating the bones, background, and teeth interference.

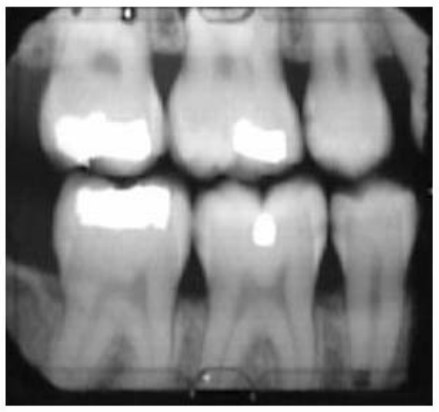

a

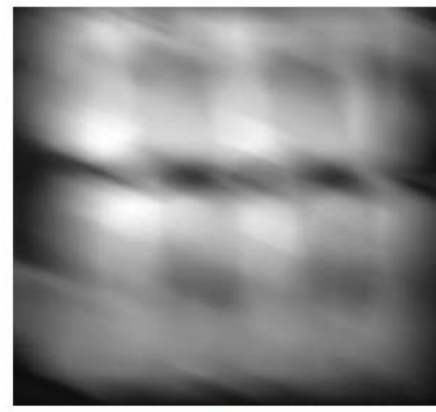

b

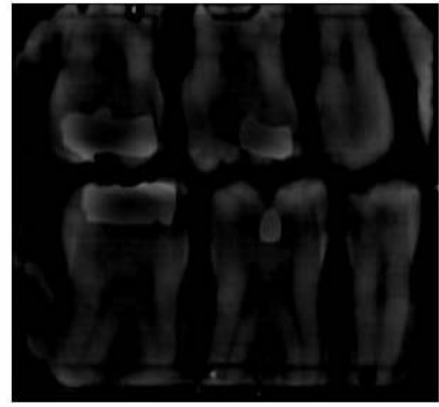

C

Figure 47: (a) Original image, (b) blurred image, (c) image resulted from subtracting the blurred image form its original

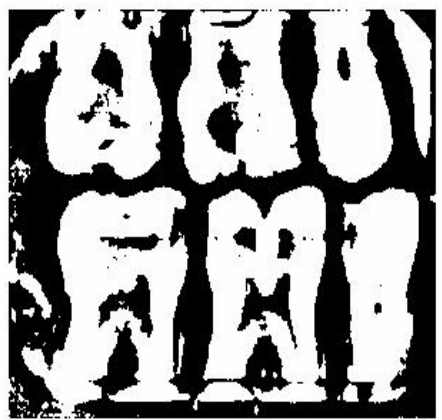

a

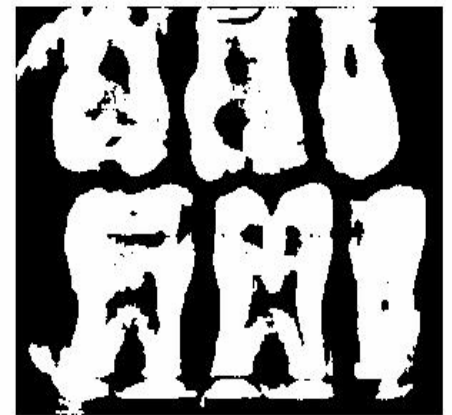

b

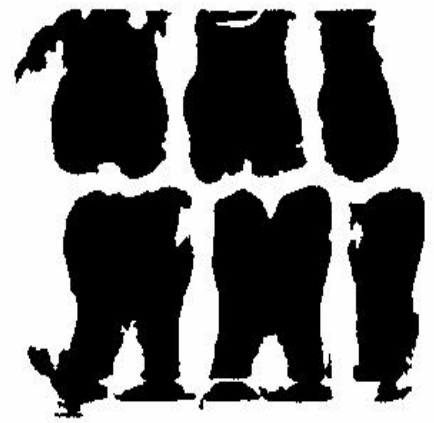

C

Figure 48: (a) Thresholded image, (b) thresholded image after filtering the small regions, (c) complement of the thresholded image.

Thinning process can be used to skeletonize the foreground in the binary image. It is defined by subtracting the hit-or miss transformation of the image from itself. In our case, the foreground of the complement of the thresholded image is corresponded to bones, teeth interfering and background in the threshold or original image. Therefore, the thinned image will divide the dental images into teeth regions or separate the neighbor teeth. Figure 49 shows the thinned image of the complement of the thresholded image. It is obvious that the 
thinned image separated the dental image into several regions; each one belongs to one tooth. The later stages of this approach including connected components labeling and refinement are the same as the first one. In this approach, we avoid choosing appropriate value for thresholding. We instead set the pixels that equal to zeros to zero and one to the other values.

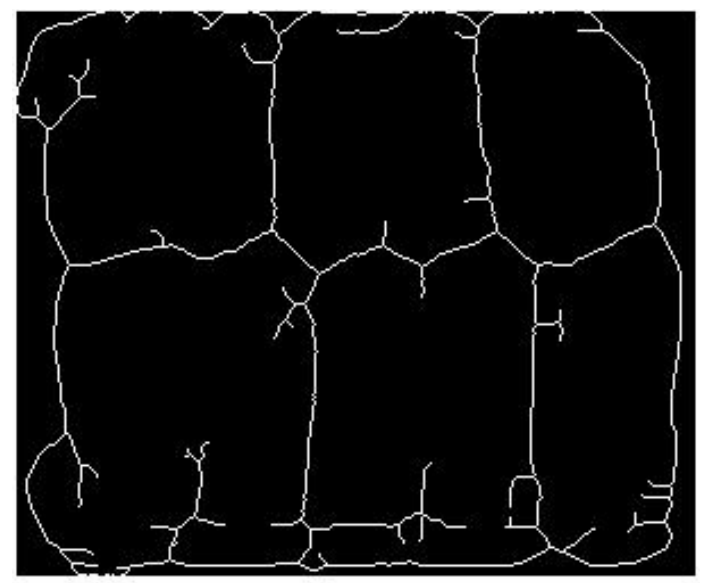

Figure 49: Thinned image

Figure 50, Figure 51, and Figure 51 show some samples of images that fully segmented successfully, partially segmented successfully, and totally failed to give any proper ROI respectively.
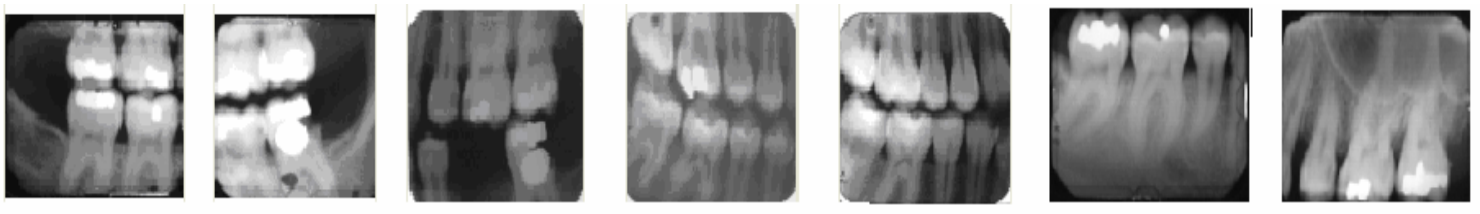

Figure 50: Examples of completely succeeded images.
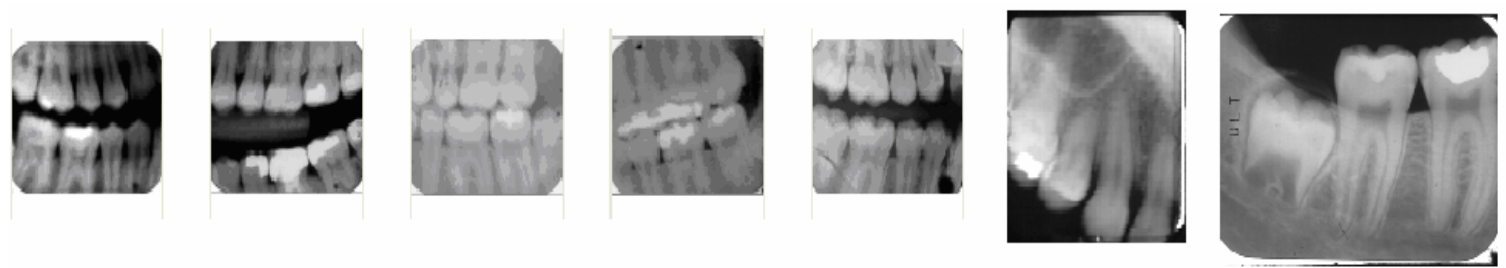

Figure 51: Examples of partially succeeded images. 

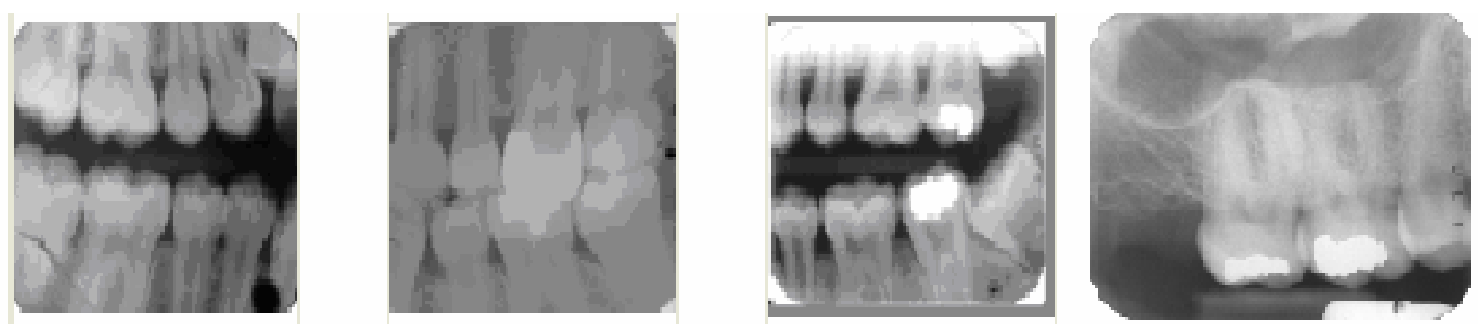

Figure 52: Examples of completely failed images.

In order to compare the performance of this approach with other approaches, we apply it on the same test sets of bitewing and periapical images that used for testing in section 5.3. Testing results of the algorithm we propose in this section for bitewing and periapical radiographic are shown in Figure 53 and Figure 54 respectively.

Figure 55 shows a graphical comparison for bitewing dental radiographs using the metrics in [84] between the dental film segmentation algorithm we proposed in this section, the proposed algorithm before and after enhancement presented in section 5.2, and the analogous algorithms proposed in [8][9], and [10]. Figure 56 shows a graphical comparison for periapical dental radiographs between the dental film segmentation algorithm we proposed in this section, and the proposed algorithm before and after enhancement presented in section 5.2.

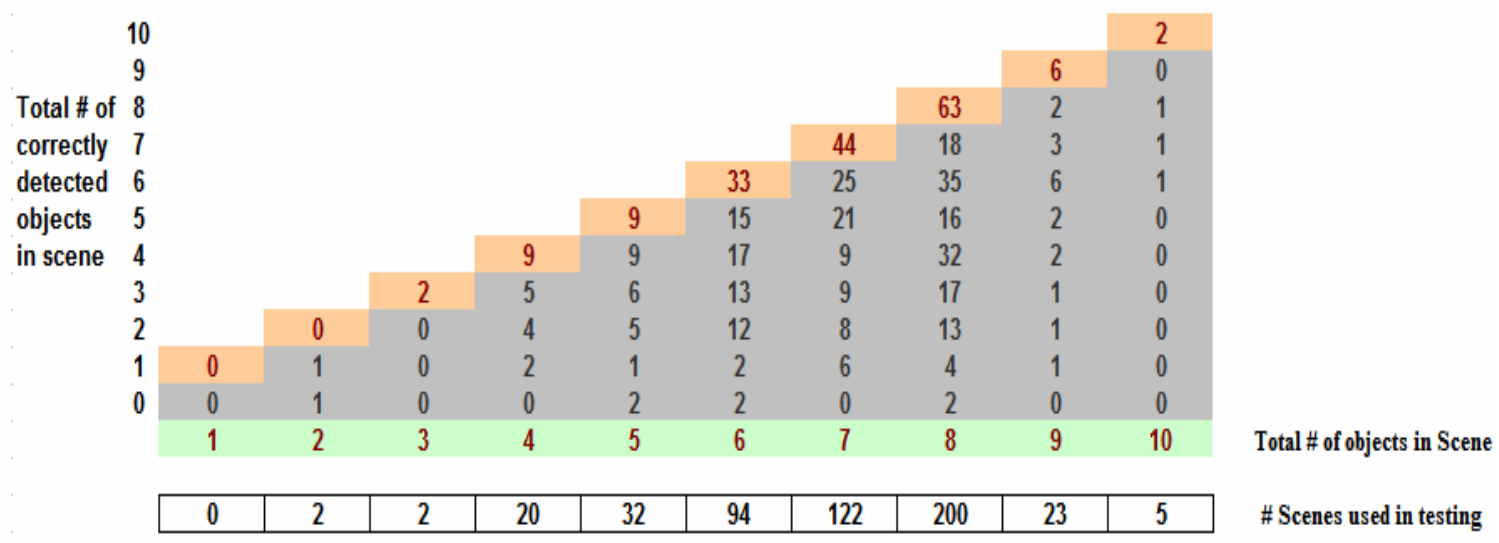

Figure 53: Testing results of our advanced teeth segmentation algorithm with bitewing views. 


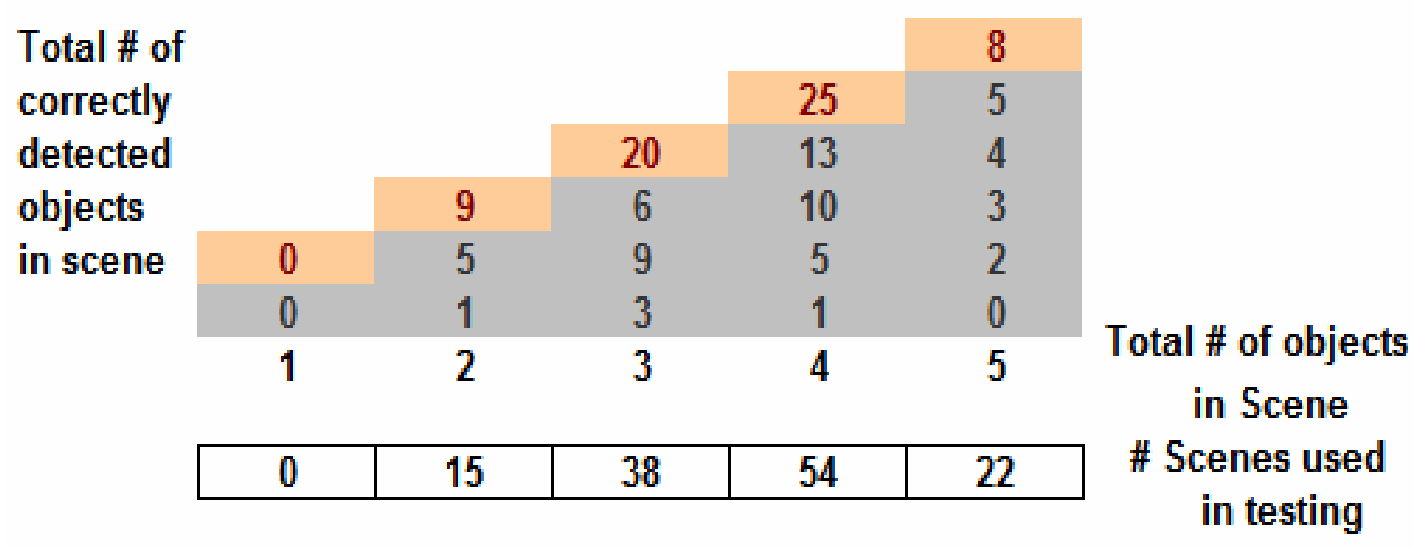

Figure 54: Testing results of our advanced teeth segmentation algorithm with periapical bitewing views.

We can conclude the following from the performance comparison:

- The optimality of the advanced approach is superior to other automated algorithms for both periapical and bitewing views..

- Failure rate of the advanced approach is the lowest for both periapical and bitewing views.

- The optimality of the advanced approach is double the one proposed in section 5.2 for periapical and bitewing views.

- In the advanced approach, more than $63 \%$ and $84 \%$ of the cases lie between the optimality and the second order optimality for bitewing and periapical views respectively.

- In the advanced approach, more than $85.5 \%$ of the cases lie between the optimality and the forth order optimality in the bitewing views. 


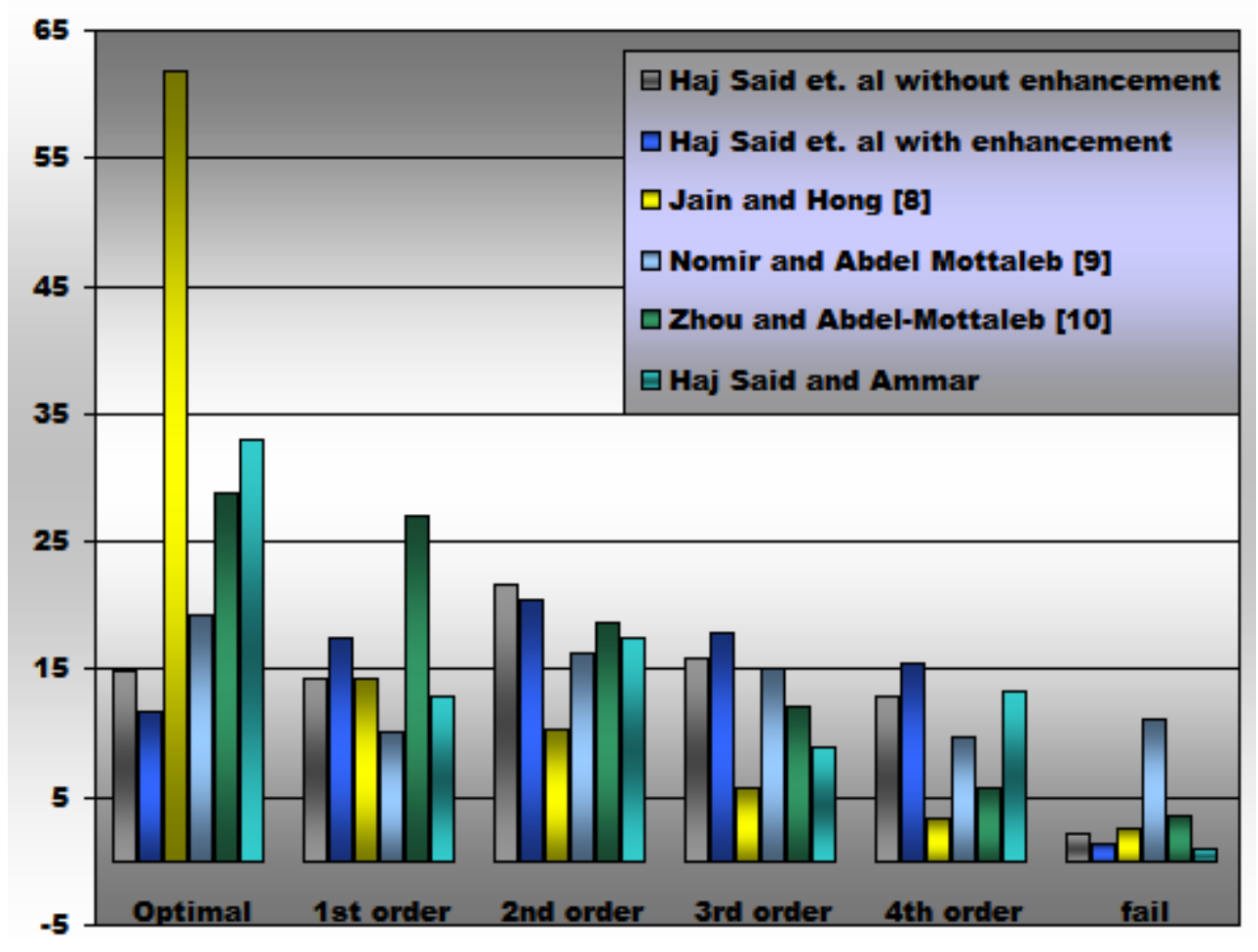

Figure 55: Performance comparison between bitewing segmentation algorithms.

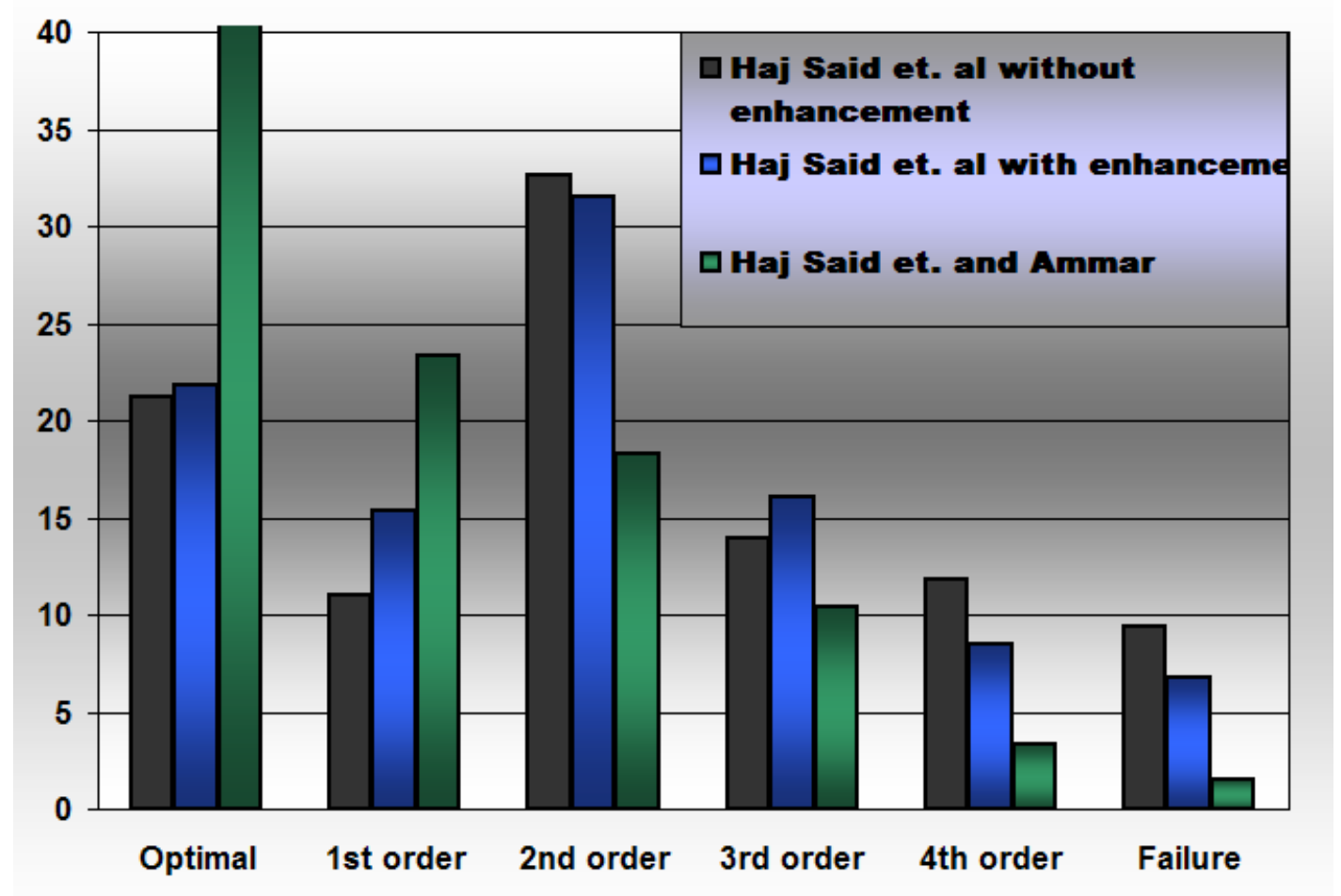

Figure 56: Performance comparison between segmentation algorithm proposed in section 5.2 and the one presented in section 5.6 for periapical views. 
We expand the test sets that used for studying the performance of this approach. Therefore, we apply it on the three test sets of 800 bitewing images, 333 upper jaw periapical images, and 321 lower jaw periapical images. Testing results of the algorithm we propose in this section for bitewing and both views of periapical dental radiographic are shown Figure 57, Figure 58, and Figure 59 in respectively.

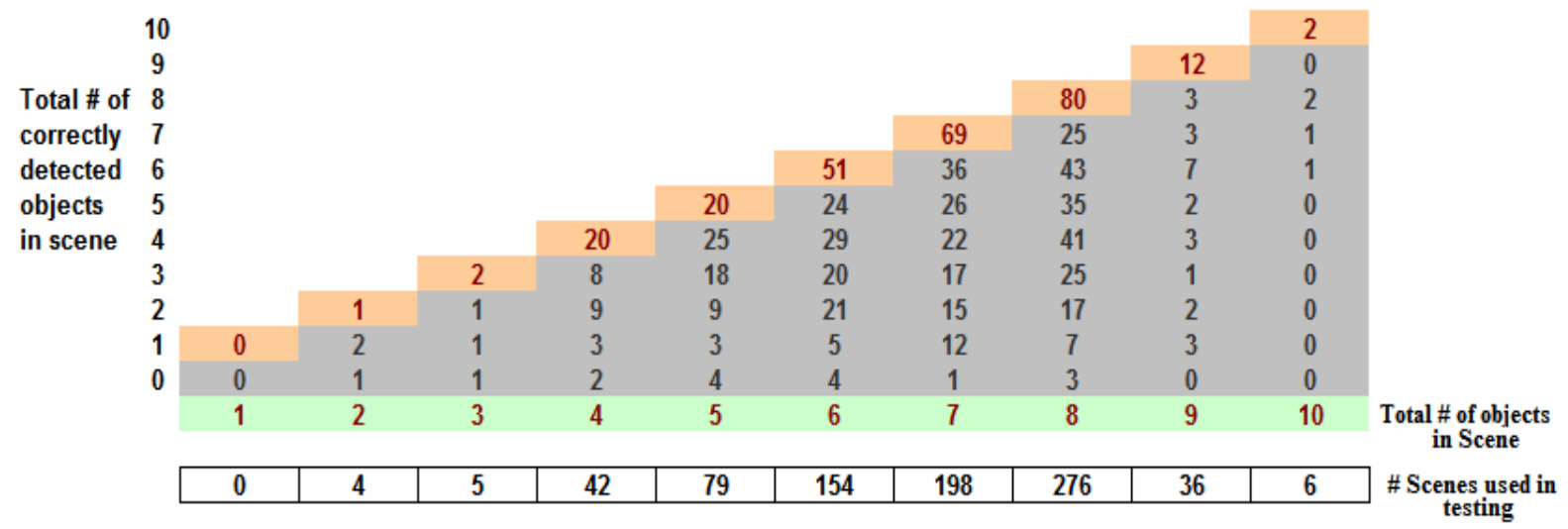

Figure 57: Testing results of our advanced teeth segmentation algorithm with 800 bitewing images views.

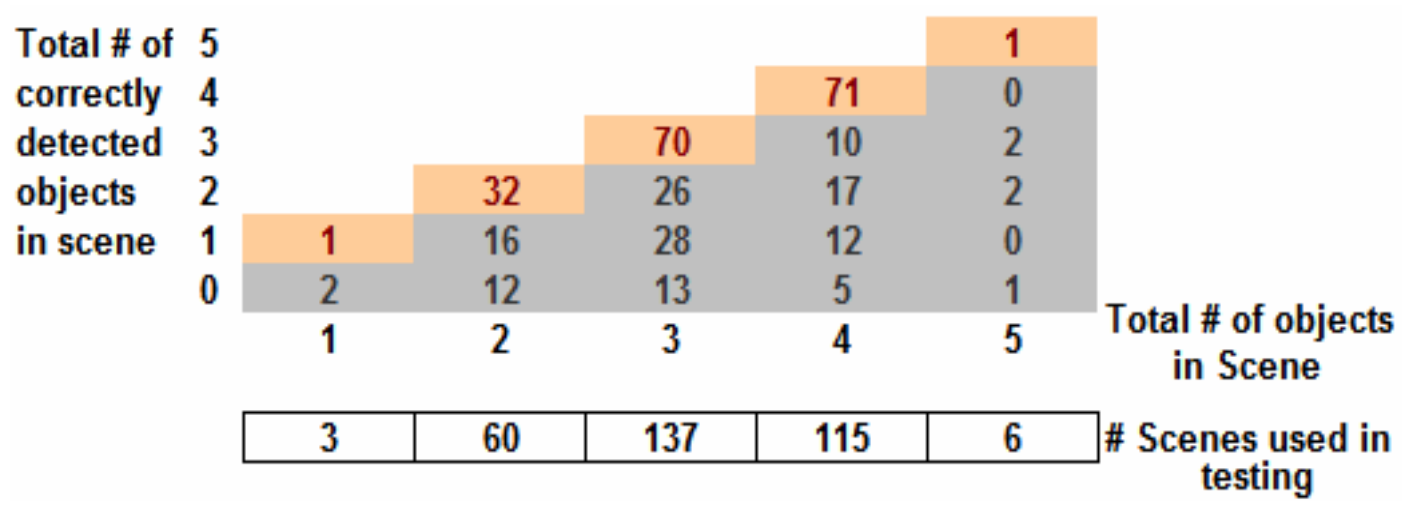

Figure 58: Testing results of our advanced teeth segmentation algorithm with 333 upper periapical images views 


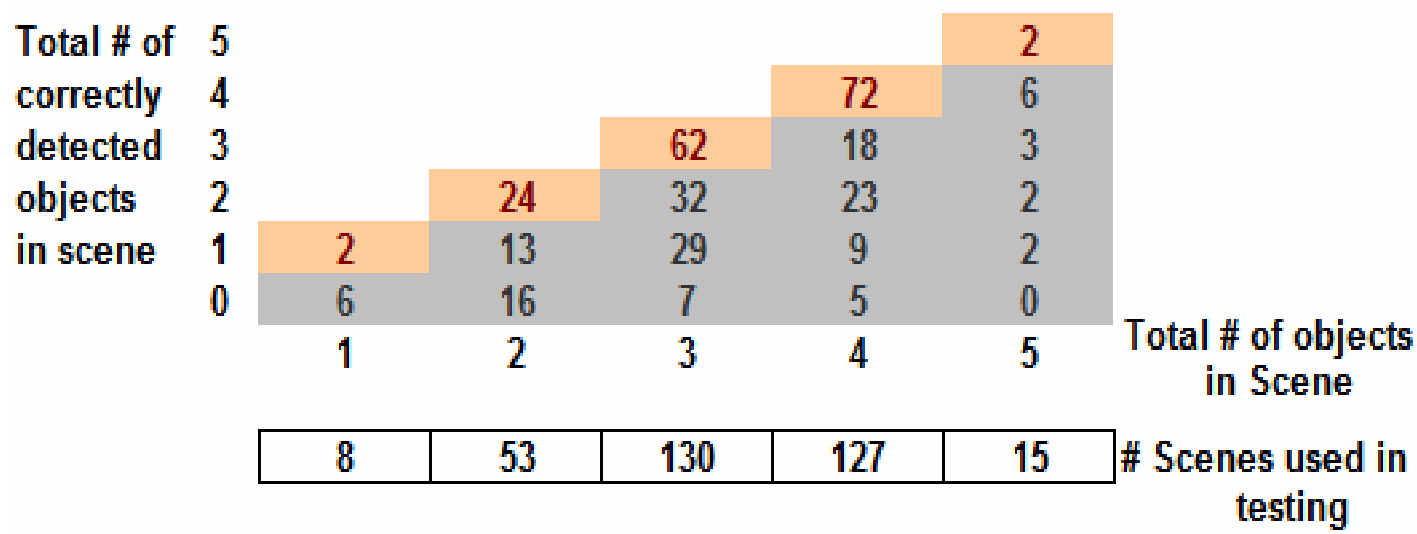

Figure 59: Testing results of our advanced teeth segmentation algorithm with 321 lower periapical images views.

Table 8 shows the performance of this approach using the metrics in [84] for larger database of bitewing, upper periapical, and lower periapical views. It is obvious that we increase the optimality of periapical views to more than $50 \%$, and we decrease the failure rate. Overall, the performance of this approach is the superior among the other automated approaches.

Table 8: Percentage of optimality, failure, and sub-optimality of our advanced teeth segmentation for various dental images views.

\begin{tabular}{|c|c|c|c|}
\cline { 2 - 4 } \multicolumn{1}{c|}{} & Bitewing & Upper Periapical & Lower Periapical \\
\hline Optimality\% & 31.14 & 57.38 & 49.49 \\
\hline $\begin{array}{c}1^{\text {st }} \text { order } \\
\text { optimality\% }\end{array}$ & 12.93 & 9.8 & 16.96 \\
\hline $\begin{array}{c}2^{\text {nd }} \text { order } \\
\text { optimality\% }\end{array}$ & 15.63 & 15.93 & 18.13 \\
\hline $\begin{array}{c}3^{\text {rd }} \text { order } \\
\text { optimality\% }\end{array}$ & 12.31 & 10.53 & 7.2 \\
\hline $\begin{array}{c}4^{\text {th }} \text { order } \\
\text { optimality\% }\end{array}$ & 12.6 & 0 & 1.89 \\
\hline Failure\% & 1.29 & 6.3 & 4.3 \\
\hline
\end{tabular}

\subsection{Summary}

Automated image segmentation in the biometric systems is essential for guiding the 
identification process. In this chapter, we have presented an automated image segmentation algorithm that handles two different types of biometric images, the dental radiograph images and facial images to extract the teeth and ear respectively. Our approach is based on Mathematical Morphology. Although the two kinds of images - radiograph images and visual graphs- are different in their properties, but the approach works well for both kinds.

The proposed algorithm includes:

1- Enhancement to emphasize the desired objects and suppress the undesired ones. In dental images, we emphasize the teeth and suppress bones, background, and teeth interfering. In the facial images, we emphasize the ear and suppress the other objects in the face

2- Thresholding to isolate the teeth/ear from the background.

3- Analyzing connected components labeling to determine the proper ROIs based on geometrical properties.

We also presented another automated approach for teeth segmentation to enhance the segmentation performance. The approach is based on performing convolution operations using point spread function and using different mathematical morphology operations to improve the isolation between the teeth. The later stages of this approach - connected components labeling and refinement- are the same as the first one.

We presented performance comparison between variants of the dental image segmentation In dental image segmentation, the main advantages of our approaches over the work introduced in $[8][9][10]$ are that:

1- They can deal not only with bitewing as other approaches but also with periapical images that represent more than $70 \%$ of the standard dental record.

2- The two proposed algorithm have the lowest failure rate in term of the segmentation result.

3- The second approach has the highest optimality among the other automated approaches for the dental bitewing images.

4- The second approach has higher optimality than the first approach before and after enhancement for the periapical views.

However, the algorithm proposed in [8] has the highest optimality, but it is still semi automated algorithm and its performance is sensitive to the manually selected initial valley 
gap point.

In ear segmentation, the main advantages of our approaches over is its ability to work with different views, angles, height, background, genders, races, and it worked properly with three different databases. The percentage of the success exceeds $90 \%$ of the images, and no subject has failed to give any successful segments. 


\section{Chapter 6: Approach for Automated Segmentation Evaluator ASE}

There is hardly no single segmentation technique that performs well for all kind of images, in addition, the performance of segmentation techniques is not the same and may vary from image to image in the same application. The problem of evaluating the segmentation outcome as proper or improper segment without human interaction or without presence of manually segmented reference image for comparison task is a difficult one.

In this chapter, we present an approach for fully ASE of the segmentation outcomes presented in the previous chapter. As we mentioned that the outcome of segmentation may not contain the proper segment or the proper ROI. Passing improper segments through the biometric systems may affect the performance of the system and waste computing resources on images that will not produce trusted identification results. Therefore, we need to implement a real time ASE that recognizes the proper and improper segments in order to reduce the segmentation error. Figure 60 shows the main goal of ASE which is to only pass the proper segments and drop the improper ones. We apply this approach on evaluating the outcomes of teeth and ear segmentation.

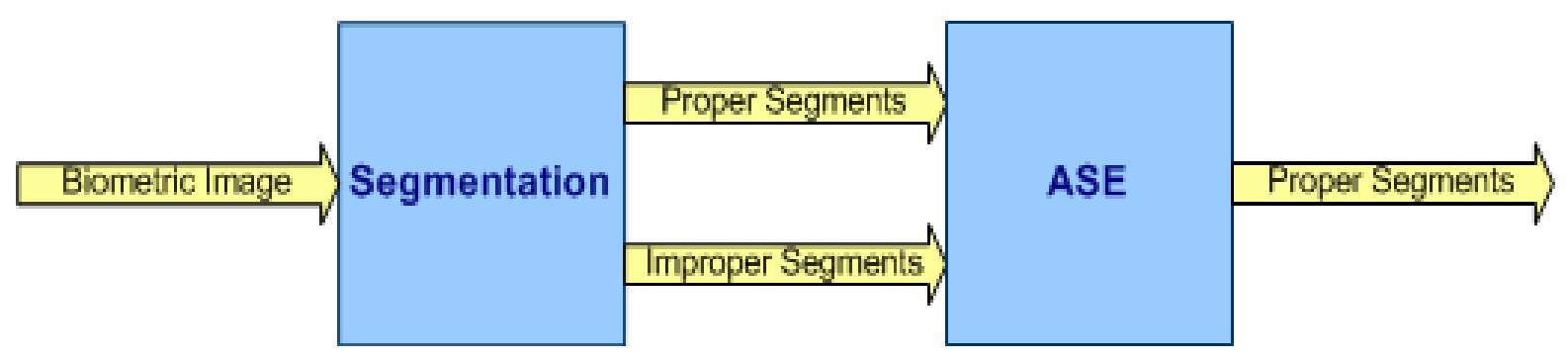

Figure 60: Automated Segmentation Evaluator ASE

\subsection{Overview:}

Our approach for implementing ASE depends on low computational-cost and appearancebased features. As illustrated in Figure 61, two stages are involved in our approach, the offline and on-line. In the off-line stage, we first classify all possible segmented outcomes from our segmentation approach into many sub-classes based on their common features, similarity, and if the segmented outcomes is proper or improper segments. These sub-classes rely not 
only on the type of biometric systems but also on the approach used for segmentation process.

For each sub-class of the segmentation outcomes, we need to choose sufficient and representative training set to be used in the training process. The next step is to standardize the view of the segmentation outcome in terms of scale and rotation. Finally, we create the Eigen-spaces corresponding to the chosen training sets. The process of the off-line stage of ASE is shown in Figure 61 (a).

In the on-line stage, we automatically evaluate the outcome of segmentation outcome online. We first normalize the segmentation outcome in the same way in the off-line stage, and then we project the segmentation outcome onto the Eigen-spaces obtained from the off line stage. Finally we used the evaluation scheme in order to determine if the outcome is proper or improper segment. The process of the on-line stage of ASE is shown in Figure 61 (b). We apply the proposed approach on two different biometric systems; the first one is evaluate the outcome of dental x-ray image segmentation, and the other one is evaluate the outcome of ear segmentation. The segmentation approach that used as pre-stage of evolution is the one presented in the previous chapter.

In section 6.2 we describe the manual classification of the segmentation outcomes into different sub-classes and the construction of the training sets. In section 6.3 we describe the view normalization process for dental and ear image segmentation outcome. In section 6.4 we describe the construction process of Eigen-spaces for each sub-class of the training sets on basis of the principle components analysis PCA. In section 6.5, we present the evaluation scheme to decide whether the segmentation outcome is proper segment or improper segment. After that, in section 6.6 and section 6.7 we report our experiment results of ASE for teeth segmentation and ear segmentation. Finally we conclude this chapter and discuss the results of our approach in section 6.8 .

\subsection{Manual Classification of the segmentation outcomes:}

In order to evaluate the segmentation outcomes, we need first to classify the segmentation outcomes into sub-classes based on their common features, similarity, and if they are proper or improper segments. After that we choose sufficient and representative samples of each of the subclasses in order to create training sets that used in the subsequence steps in the 
evaluation process. However, the training sets are highly affecting the classification process because the results will be biased to the training sets.

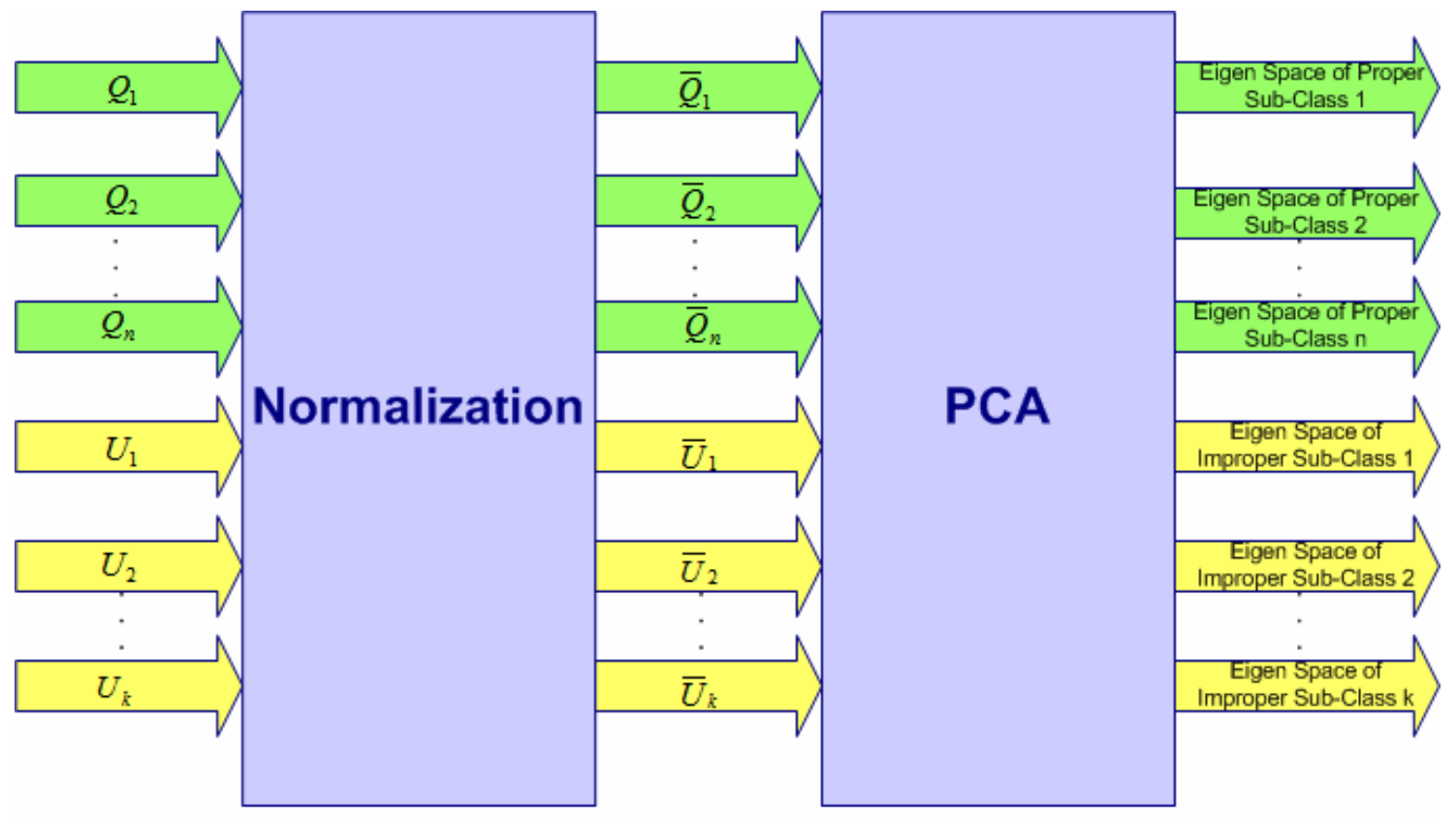

(a)

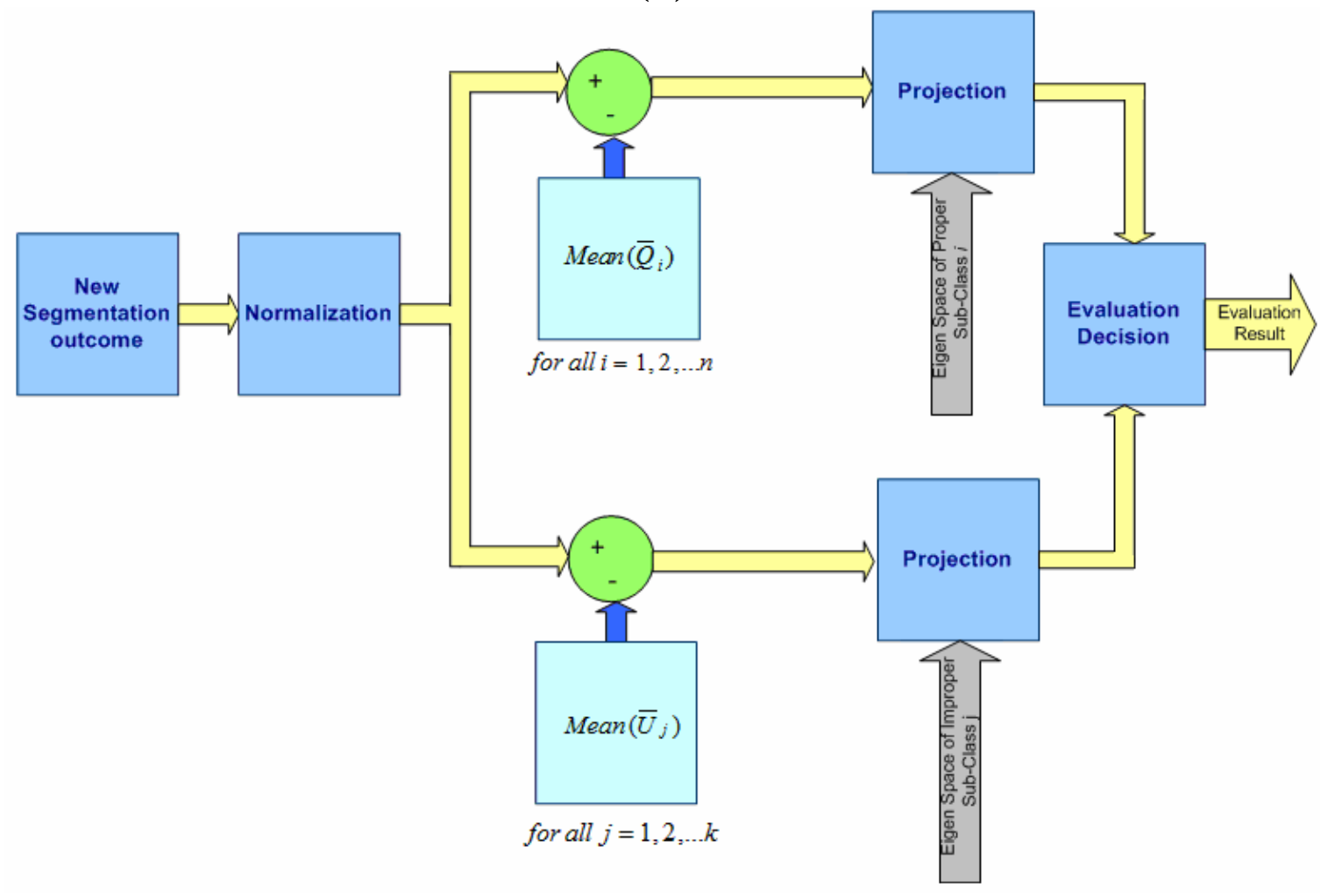

(b)

Figure 61: (a) off-line stage of ASE. (b) on-line stage of ASE. 
In the following subsections, we will show the main sub-classes used in evaluation process for the dental image segmentation and ear segmentation.

\subsubsection{Dental Image Segmentation Sub-classes}

We classify the segmentation outcome of dental images into seven sub-classes in the bitewing images, four subclasses in the upper periapical images, and four subclasses in the lower periapical images. In Table 9, we present the sub-classes of segmentation outcomes of bitewing, upper periapical, and lower periapical. The classification is based on i) location of the tooth in the upper or lower jaw, ii) if the segmentation outcome is tooth, undersegmentation, oversegmentation, or non tooth object, iii) the dental film type (bitewing, upper periapical, or lower periapical) . Figure 62- Figure 68 depict a sample of exemplars from each subclass from bitewing dental images segmentation. Figure 69-Figure 71 and Figure 72-Figure 75 depict a sample of exemplars in each subclass from upper and lower periapical dental images segmentation respectively.

Table 9: Classification of teeth segmentation outcomes for various views of dental x-rays radiographs, bitewing, upper periapical, and lower periapical

\begin{tabular}{|c|c|c|c|}
\hline Subclass & Bitewing & $\begin{array}{c}\text { Upper } \\
\text { Periapical }\end{array}$ & $\begin{array}{c}\text { Lower } \\
\text { Periapical }\end{array}$ \\
\hline $\begin{array}{c}\text { Upper Jaw } \\
\text { Tooth }\end{array}$ & $\mathrm{X}$ & $\mathrm{X}$ & $\mathrm{N} / \mathrm{A}$ \\
\hline $\begin{array}{c}\text { Lower Jaw } \\
\text { Tooth }\end{array}$ & $\mathrm{X}$ & $\mathrm{N} / \mathrm{A}$ & $\mathrm{X}$ \\
\hline $\begin{array}{c}\text { Upper Jaw } \\
\text { Oversegmentation }\end{array}$ & $\mathrm{X}$ & $\mathrm{X}$ & $\mathrm{N} / \mathrm{A}$ \\
\hline $\begin{array}{c}\text { lower Jaw } \\
\text { Oversegmentation }\end{array}$ & $\mathrm{X}$ & $\mathrm{N} / \mathrm{A}$ & $\mathrm{X}$ \\
\hline $\begin{array}{c}\text { Upper Jaw } \\
\text { Undersegmentation }\end{array}$ & $\mathrm{X}$ & $\mathrm{X}$ & $\mathrm{N} / \mathrm{A}$ \\
\hline $\begin{array}{c}\text { lower Jaw } \\
\text { Undersegmentation }\end{array}$ & $\mathrm{X}$ & $\mathrm{N} / \mathrm{A}$ & $\mathrm{X}$ \\
\hline Background & $\mathrm{X}$ & $\mathrm{X}$ & $\mathrm{X}$ \\
\hline
\end{tabular}




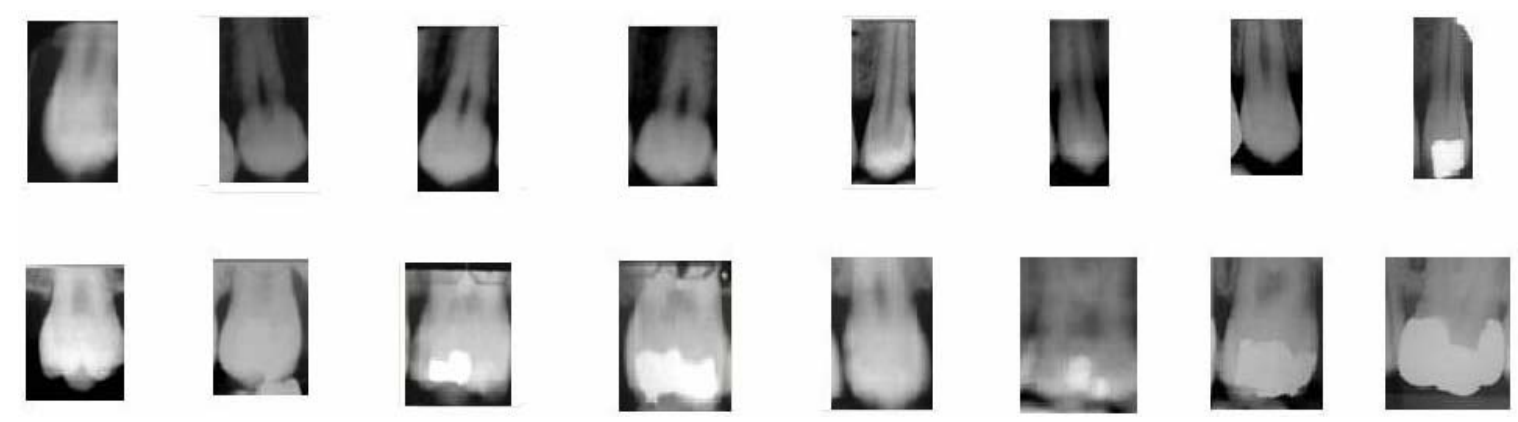

Figure 62: Samples of upper jaw teeth in the Bitewing images
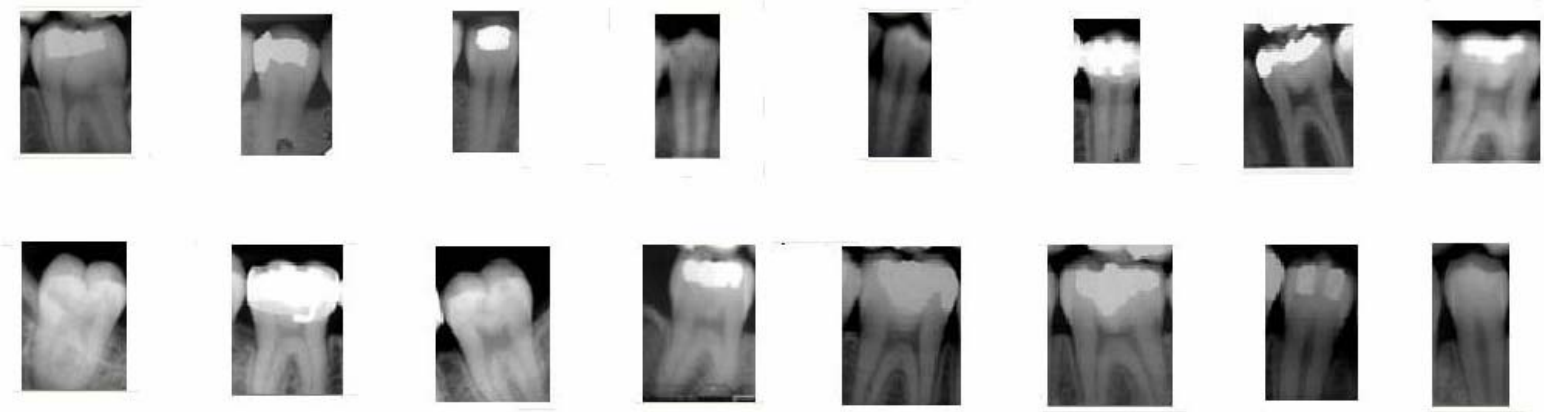

Figure 63: Samples of lower jaw teeth in the Bitewing images
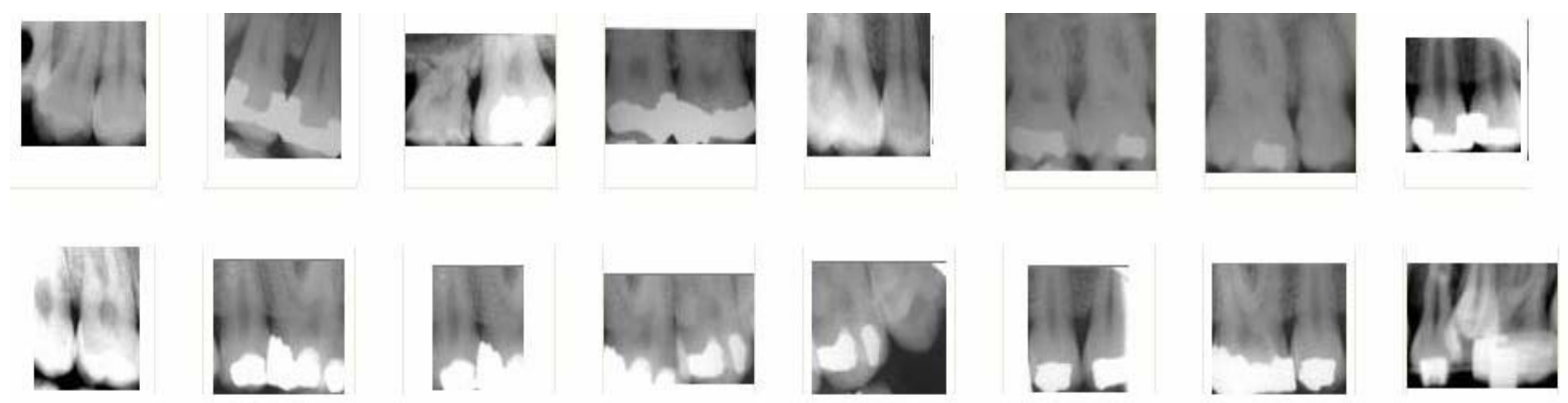

Figure 64: Samples of upper jaw oversegmentation outcomes in the Bitewing images
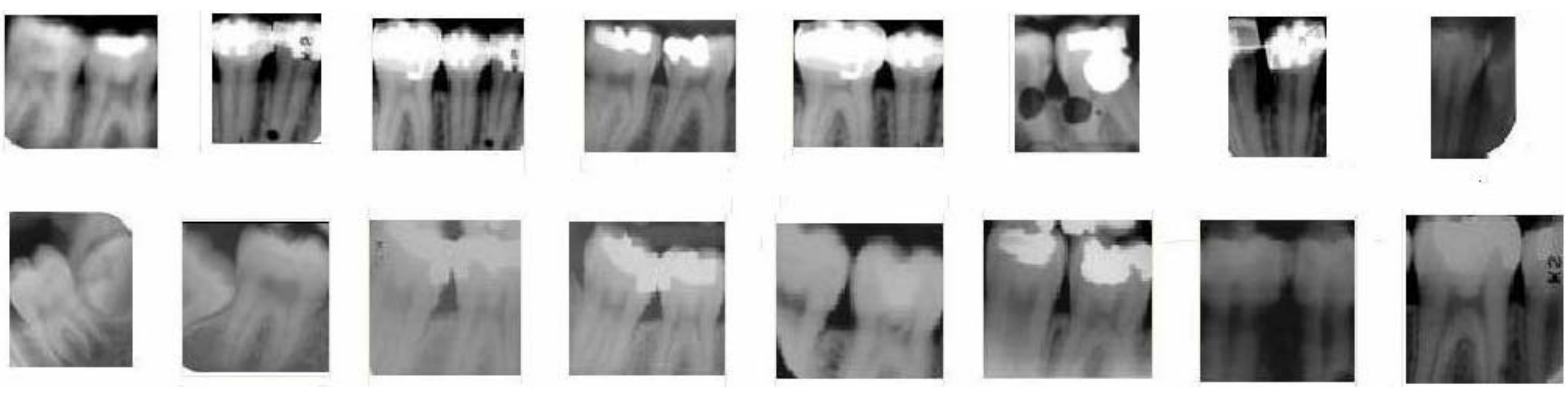

Figure 65: Samples of lower jaw oversegmentation outcomes in the Bitewing images 

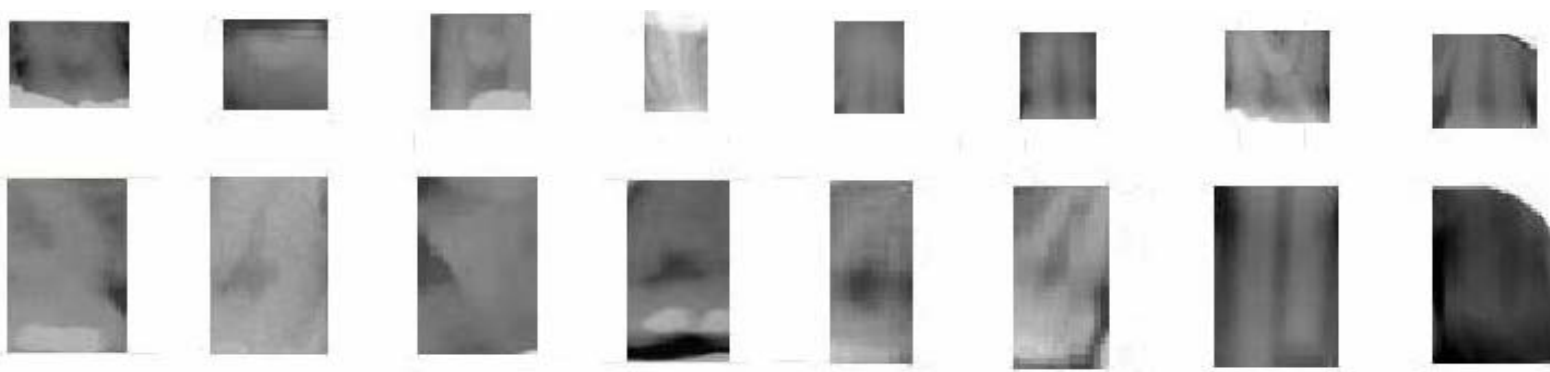

Figure 66: Samples of upper jaw undersegmentation outcomes in the Bitewing images
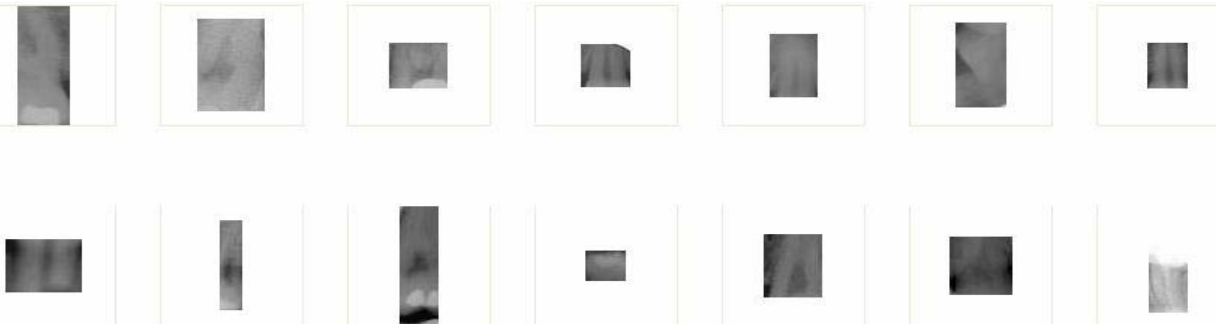

V

Figure 67: Sample of lower jaw undersegmentation outcomes in the Bitewing images
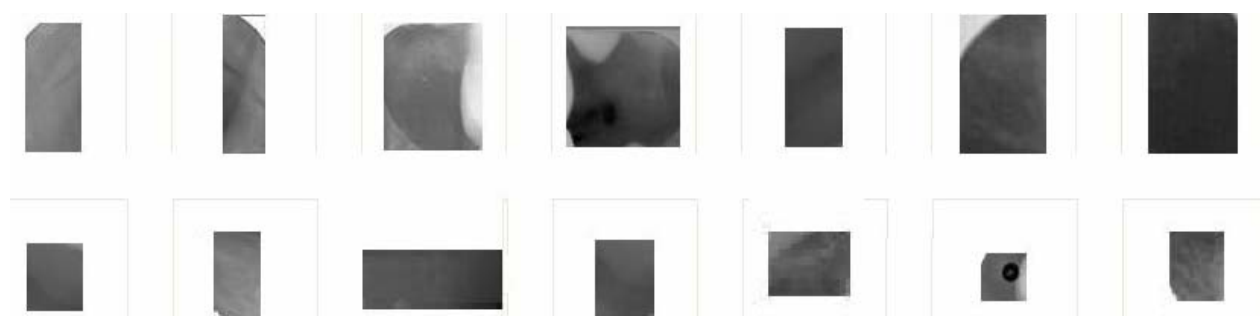

Figure 68: Samples of non-teeth/background outcomes in the Bitewing images
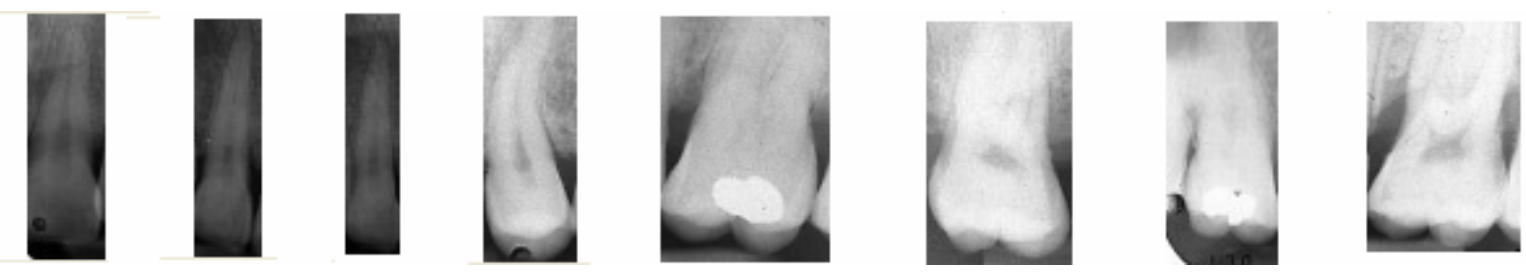

Figure 69: Samples of teeth in the upper jaw periapical images
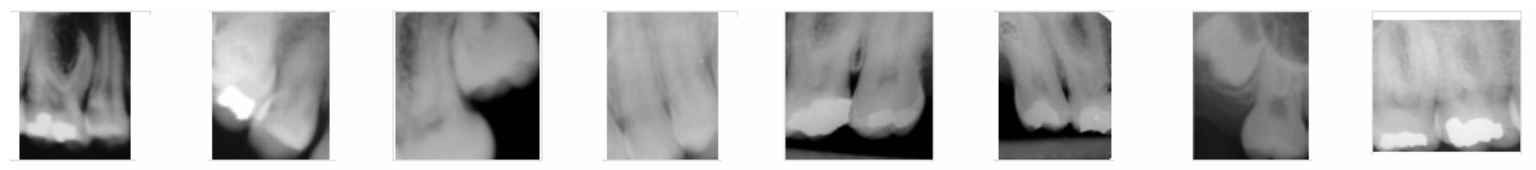

Figure 70: Samples of upper jaw oversegmentation outcomes in the upper jaw periapical dental images. 

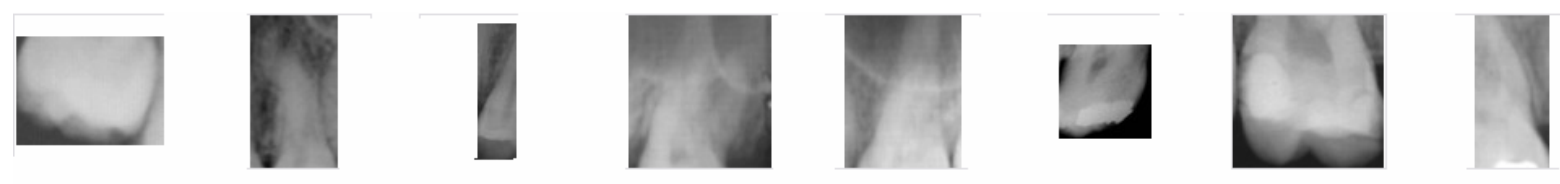

Figure 71: Samples of upper jaw undersegmentation outcomes in the upper jaw periapical dental images.
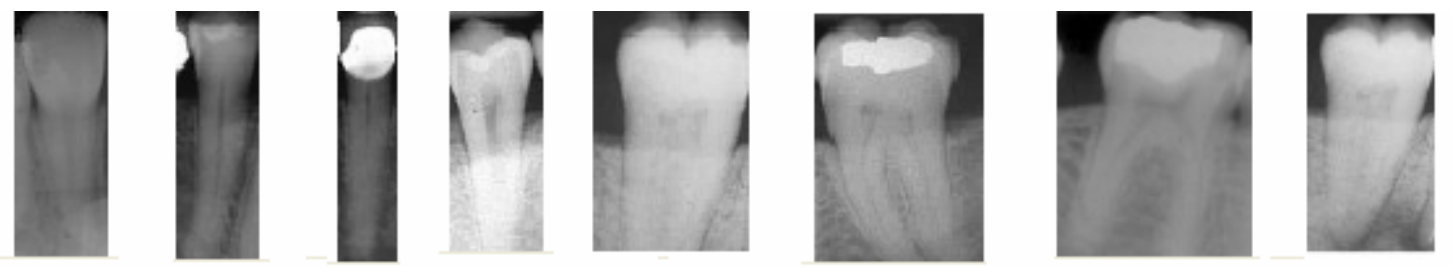

Figure 72: Samples of teeth in the lower jaw periapical images
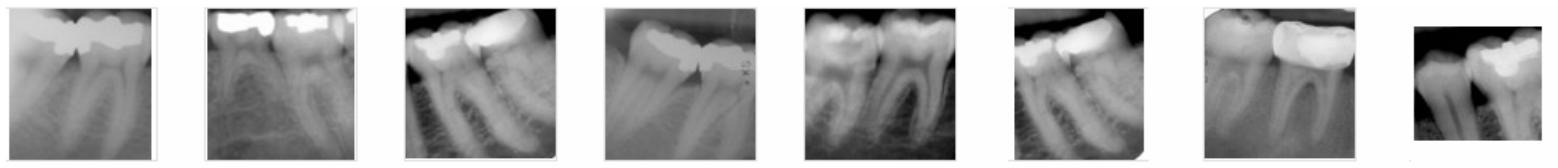

Figure 73: Samples of upper jaw oversegmentation outcomes in the lower jaw periapical dental images.
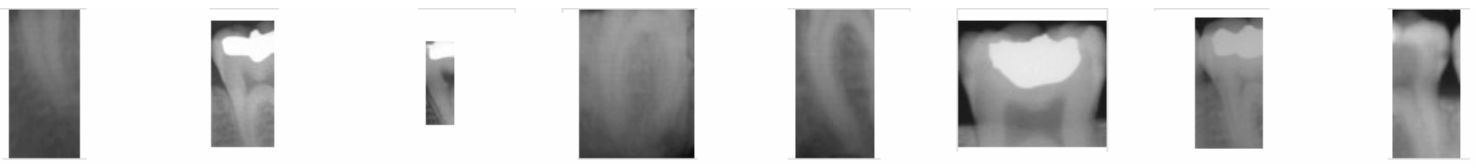

Figure 74: Samples of lower jaw undersegmentation outcomes in the lower jaw periapical dental images.
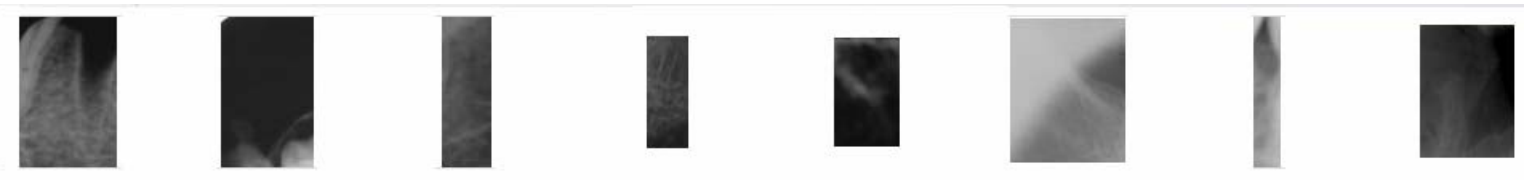

Figure 75: Samples of background outcomes in the periapical dental images.

\subsubsection{Ear image segmentation subclasses}

We classify the ear segmentation outcome of facial images into five subclasses based on the facial angle view and ear/ non ear segments. The five subclasses are:

1- Segments contain ear in an angle view between $[-10,40]$.

2- Segments contain ear in an angle view between $[41,75]$.

3- Segments contain part of the ear in an angle view between $[-10,40]$.

4- Segments contain part of ear in an angle view between $[41,75]$. 


\section{5- Non ear segments}

Figure 76-Figure 80 depict a sample of exemplars from each subclass from ear image segmentation outcomes with different views.
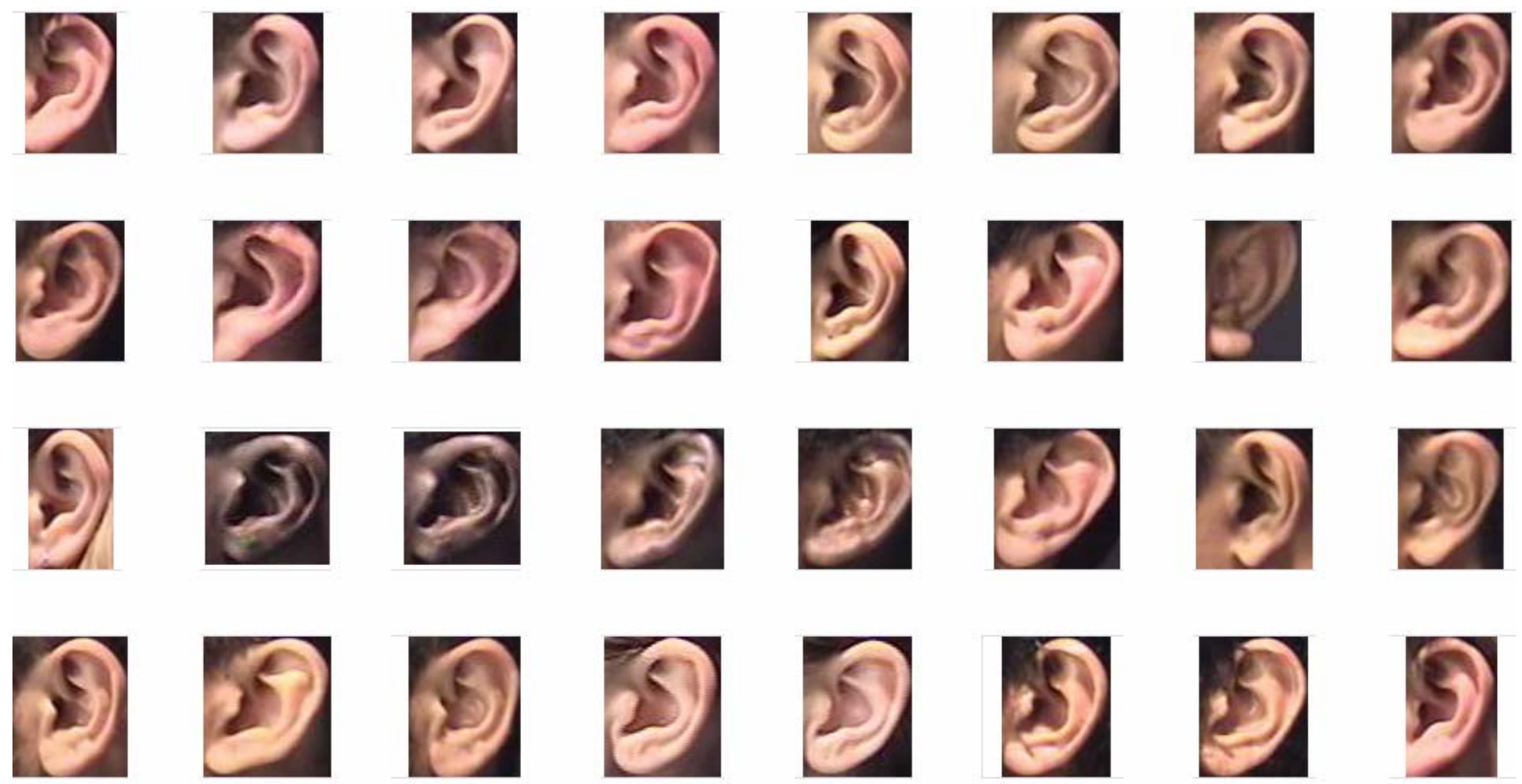

Figure 76: Samples of proper ear segmentation outcomes in a angle view between [-10, 40].
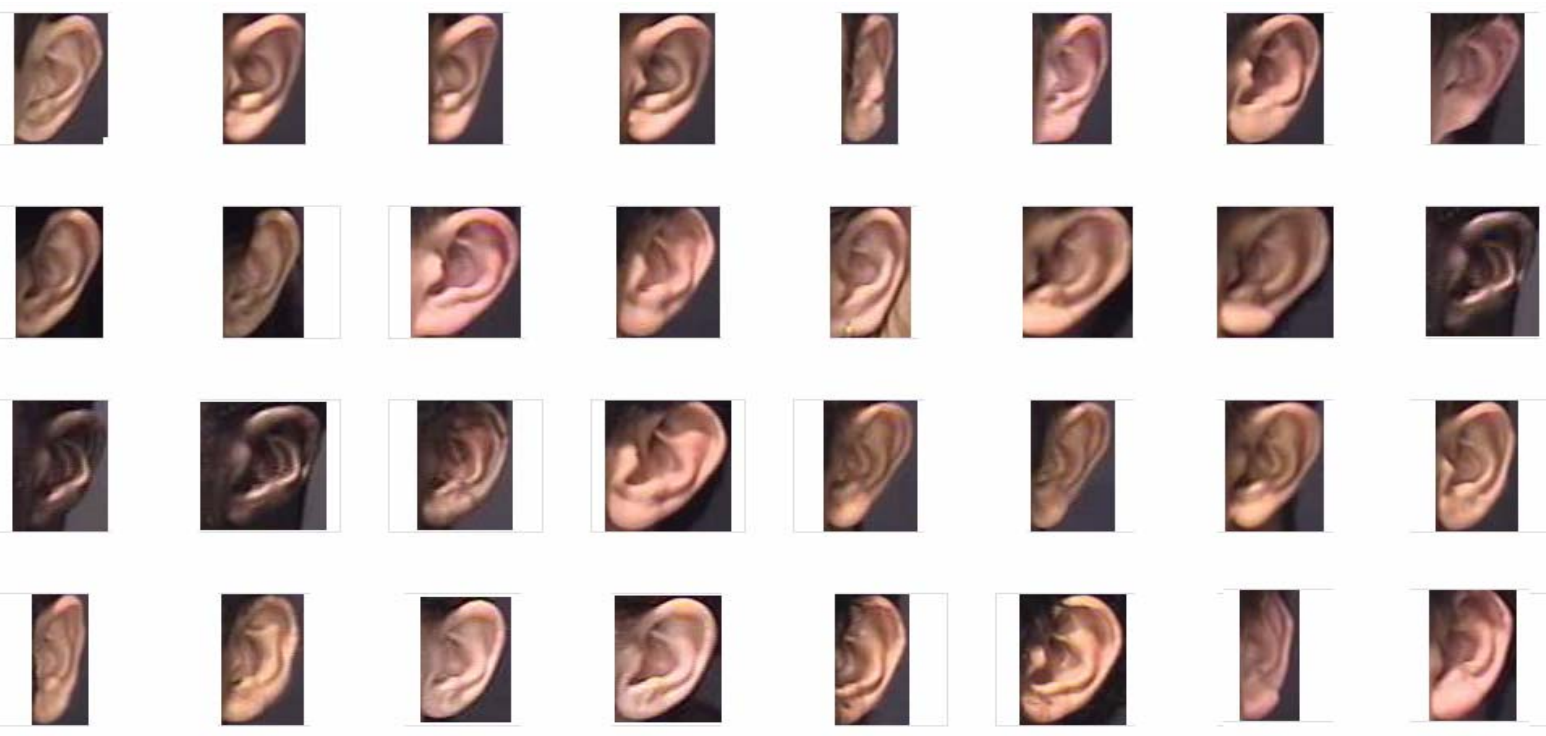

Figure 77: Samples of proper ear segmentation outcomes in a angle view between $[40,80]$. 

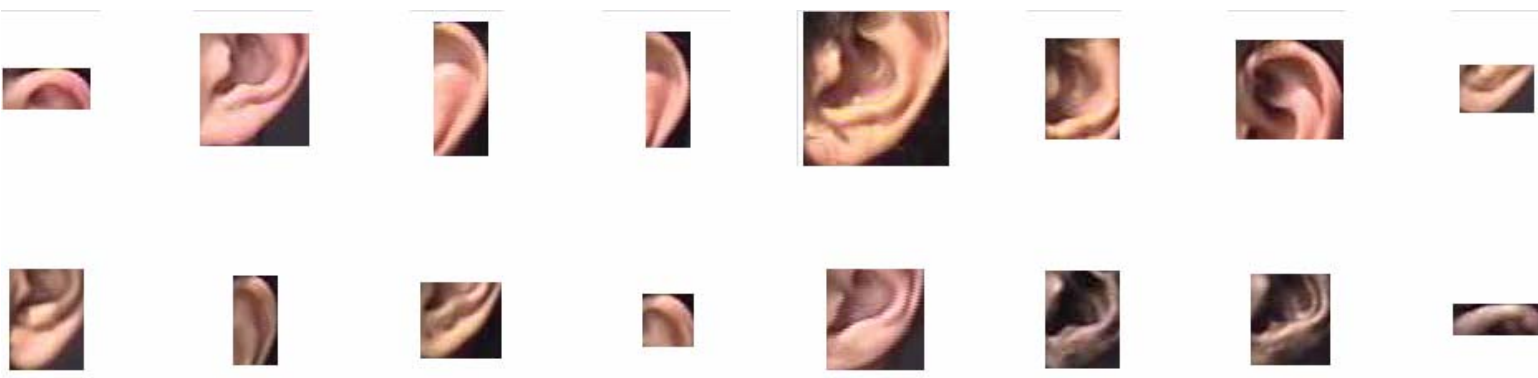

Figure 78: Samples of partially succeeded ear segmentation outcomes in a angle view between $[-10,40]$.
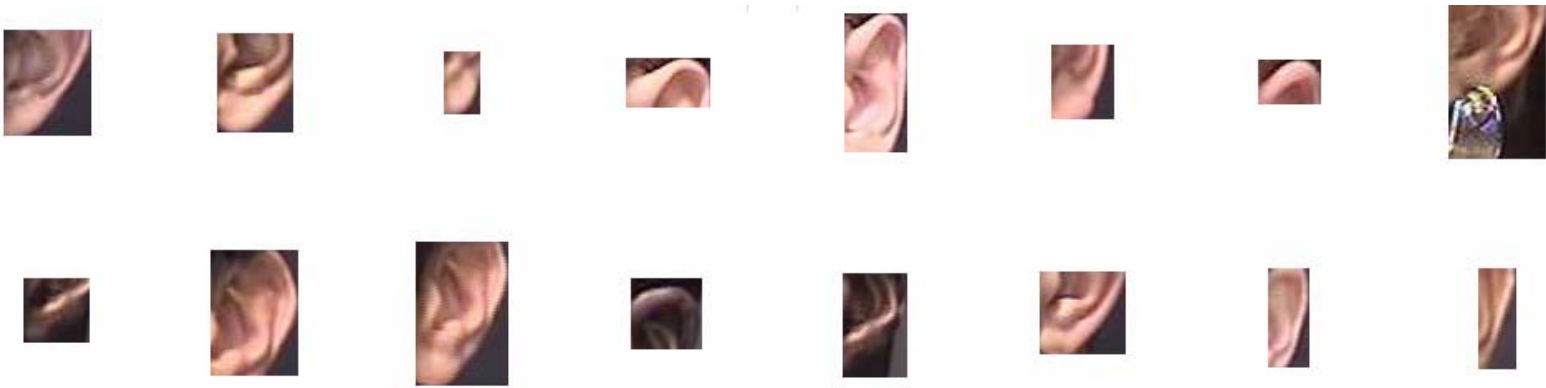

Figure 79: Samples of partially succeeded ear segmentation outcomes in a angle view between $[40,80]$.
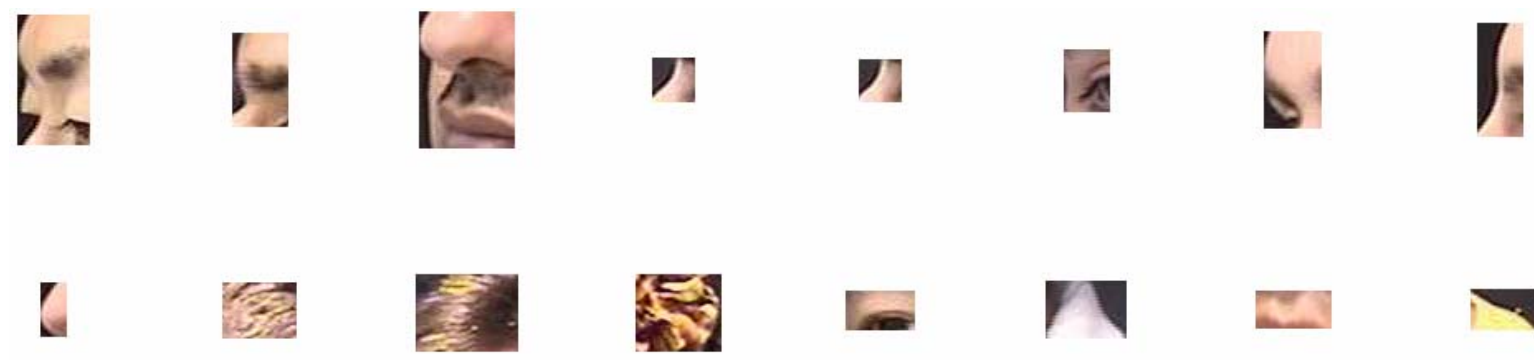

$\checkmark$
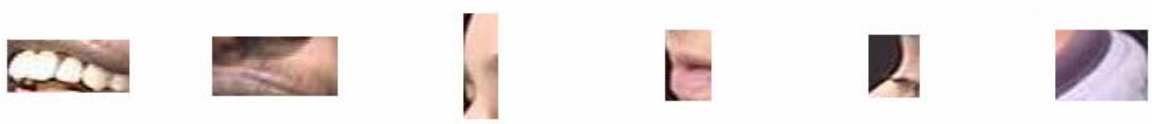

I
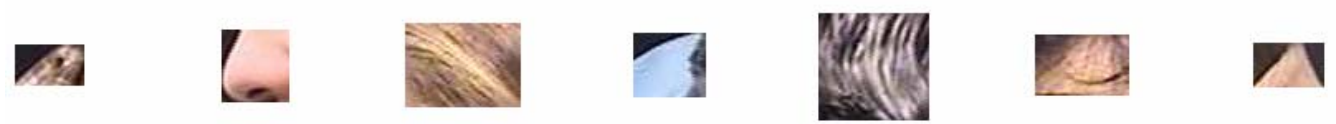

5

Figure 80: Samples of improper ear segmentation outcomes. 


\subsection{View Normalization}

While the outcomes of the segmentation approach do not have standard view in terms of scale, rotation. The view normalization is an essential step to improve the efficiency of ASE and to overcome variations in orientation and scale. It improves the training process and comply the segmentation outcomes with training sets. In other hand, in the view normalization step, we try to standardize the way we present the segmentation outcome in a standard view.

\subsubsection{Normalization of Dental Segmentation Outcomes}

One way to normalize the view of the dental segmentation outcome is to ensure that the surface of tooth crown appears horizontally as much as we can. This can be done by the finding the appropriate rotation angle that is corresponding to strongest horizontal edges in the segmentation outcome. The following steps show the view normalization task in the dental images:

1- We rotate the segmentation outcome in an angle range $\theta \in[-25,25]$ with interval one degree.

2- For each of the rotated images $I_{\theta}$ that have the size $M_{\theta} \times N_{\theta}$, we apply canny edge detection on the rotated segmentation outcome in order to produce edge image $E I_{\theta}$ as shown in Figure 81 (b) and Figure 81 (c).

3- For each $E I_{\theta}$, we group the edges based on their connectivity and then we remove the short edges.

4- We compute the horizontal integral projection of each edge image $E I_{\theta}$ :

$$
\operatorname{Int}^{\theta}(i)=\sum_{j=1}^{N_{\theta}} E I_{\theta}(i, j)
$$

where $i$ is the $i$ th row,

5- For each edge image $E I_{\theta}$, we determine the maximal $\operatorname{Int}^{\theta}(i)$ : $\max \_I n t^{\theta}=\max \left\{I n t^{\theta}(i)\right.$ for $\left.i=1 \ldots . M_{\theta}\right\}$

6- We rotate the segmentation outcome in an angle $\omega$ that is corresponding to angle that satisfies max $\left(\max _{-} I n t^{\theta}\right)$ for $\theta \in[-25,25]$. 
7- After detecting the rotation angle, we resize the segmentation outcome to $64 \times 64$ pixels.

Figure 81 shows the result of view normalization for various outcome of dental image segmentation.
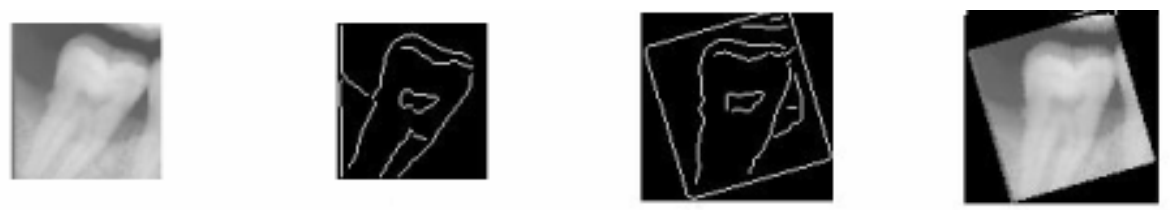

\section{Tooth}
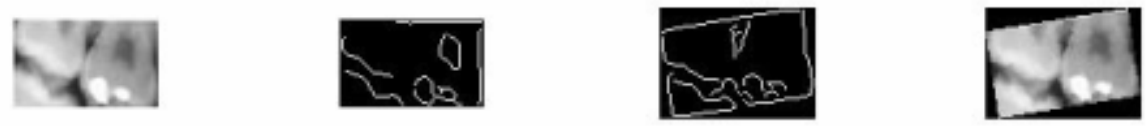

\section{Oversegmentation}
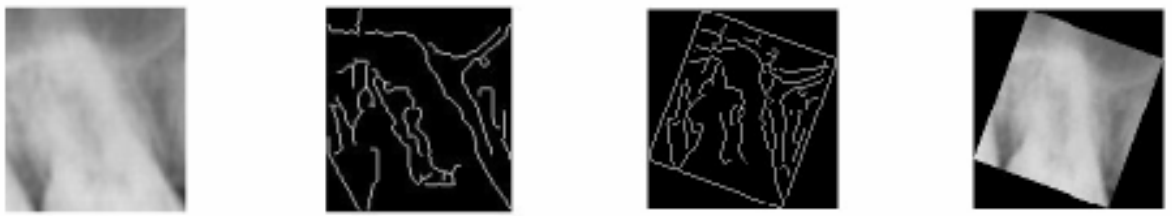

\section{Undersegmentation}

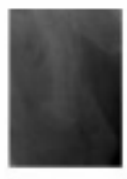

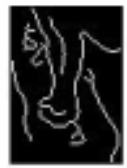

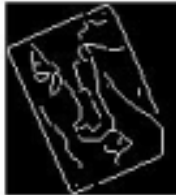

Background

(a) (b)

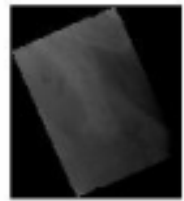

(d)

Figure 81: View normalization for different dental image segmentation sub-classes, Tooth, Oversegmentation, undersegmentation, and Background. (a) original segment, (b) edge image, (c) rotated edge image corresponding to strongest vertical edges, (d) normalized segment image.

\subsubsection{Normalization of Ear Segmentation Outcomes}

The ear orientation varies with different face view for the same subject and varies from subject to subject in the same view. Therefore; the view normalization of the ear segmentation outcomes is important to standardize the ear view. We normalize the ear segmentation outcomes by: 
1- Rotating the connected component $I_{c}$ obtained in the third step of ear segmentation in an angle range $\theta \in[-30,30]$ with interval one degree.

2- Computing the height of each rotated image $I_{c \theta}$.

3- Rotating the segmentation outcome in an angle $\omega$ that corresponds to angle which leads to highest height of $I_{c \theta}$.

4- After detecting the rotation angle, we resize the segmentation outcome to $64 \times 64$ pixels.
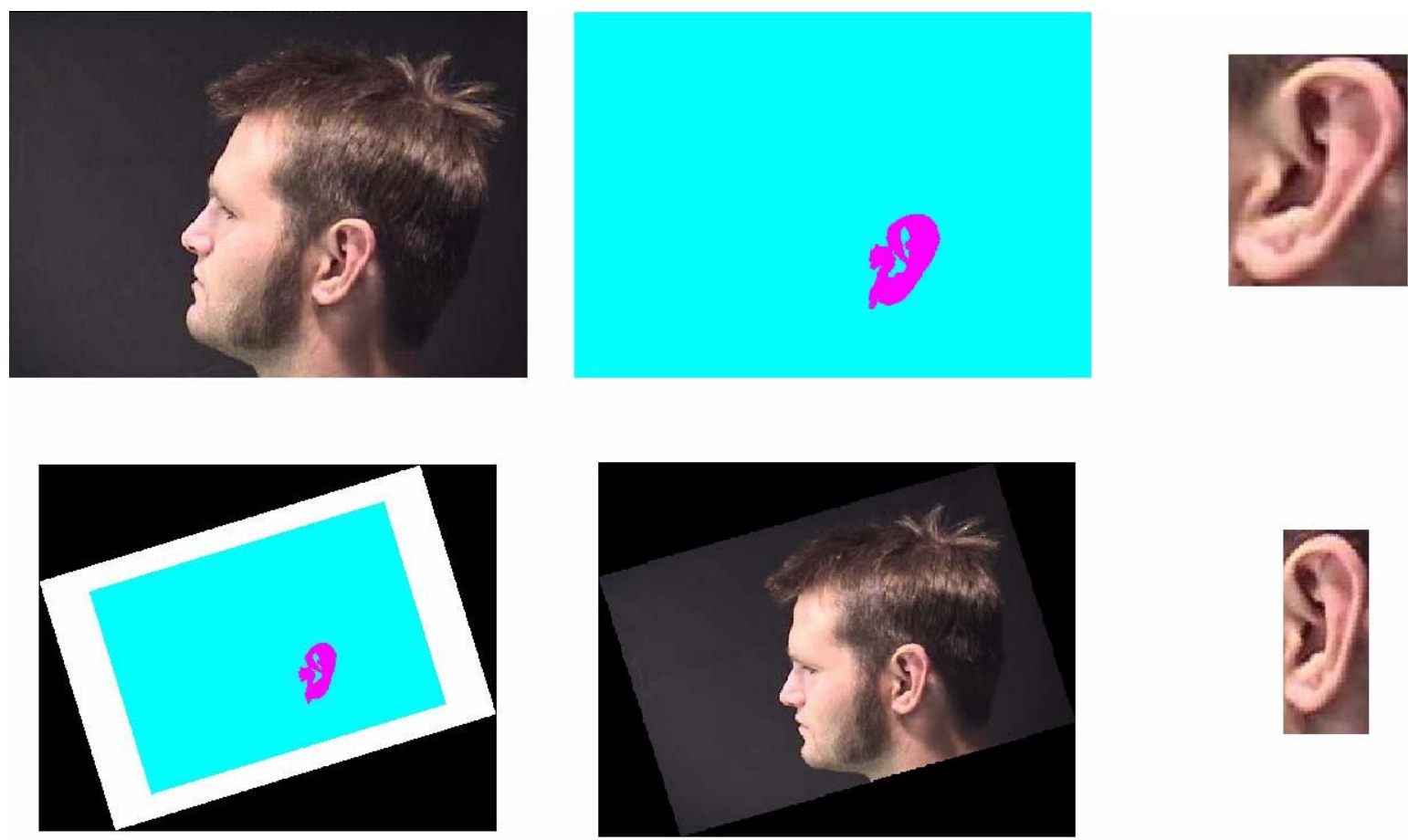

Figure 82: View normalization of ear image segmentation outcome.

\subsection{Eigen Space}

In our case, we have $64 \times 64$ pixels normalized image obtained from segmented outcome image. This generates problems of high dimensional pattern classification, memory storage, and large computational complexity. In addition, we need to detect the correlated features to reduce the dimensionality. However, expanding the number of features or increasing the dimensionality does not guarantee that the performance of classification would be better. Since the objects in each subclass are similar and have large statistical regularities, we can use the Eigen Space based method to overcome the high dimension problem. The idea of 
constructing the subclass-space of each subclass of the segmentation outcome is to capture the most common features that corresponding to the given training set of that subclass. We use the Principle Component Analysis PCA framework to construct these subclass- spaces. Let $T$ is a training set of subclass containing $\underline{T_{1}}, \underline{T_{2}}, \ldots, \underline{T_{K}}$ exemplar where $\mathrm{K}$ is the total number of exemplars in the training set for that subclass. All exemplars have been normalized and resized to $64 \times 64$ pixels in the normalization step. To construct the subclass space we do the following:

1. We compute the mean of exemplars in each of the subclass training sets:

$$
\underline{\mu}=\frac{1}{K} \sum_{i=1}^{K} \underline{T_{i}}
$$

Where $\underline{T_{i}}$ is the vector representation of ith exemplar.

2. Let $\underline{X_{i}}=\underline{T_{i}}-\underline{\mu}$. We Compute the covariance matrix $C=M^{T} M$ where $M=\left[\underline{X_{1}} \underline{X_{2}} \cdots \underline{X_{k}}\right]$.

3. While $C$ is a symmetric positive semi-definite matrix and can be decomposed to $C=V \Lambda V^{T}$ where $\mathrm{V}$ is the eigenvector matrix and $\Lambda$ is a diagonal matrix of the ordered eigenvalues.

4. We compute the principle components form a matrix $Y=M V$.

5. Of the $\mathrm{K}$ eigenvectors, we choose $\mathrm{N}$ eigenvector for classification. Choosing the eigenvectors is based on the value of eigenvalue that accounts for the variations in the training set.

\subsection{Evaluator}

After getting the Eigen space of each subclass of segmentation outcomes, we need to develop an evaluator in order to recognize whether the segmentation outcome is proper or improper. If we consider that we have $m$ sub-classes from the segmentation outcomes, and let assume that the conditional density functions for all sub-classes have normal distribution, and then we can write that the conditional density function of sub-class $w_{i}$ is: 


$$
p\left(\underline{x} \mid w_{i}\right)=\frac{\exp \left[-\frac{1}{2}\left(\underline{x}-\underline{\mu_{i}}\right)^{t} \Sigma_{i}^{-1}\left(\underline{x}-\underline{\mu_{i}}\right)\right]}{(2 \pi)^{\frac{N}{2}}\left|\Sigma_{i}\right|^{\frac{1}{2}}}
$$

Where $\mu_{i}$ and $\Sigma_{i}$ are the mean and covariance of sub-class $w_{i}$ respectively. By substituting the Mahalanobis Distance $d_{i}(x)=\left(\underline{x}-\underline{\mu_{i}}\right)^{t} \Sigma_{i}^{-1}\left(\underline{x}-\underline{\mu_{i}}\right)$ in (1)

$$
p\left(\underline{x} \mid w_{i}\right)=\frac{\exp \left[-\frac{1}{2} d_{i}(x)\right]}{(2 \pi)^{\frac{N}{2}}\left|\Sigma_{i}\right|^{\frac{1}{2}}}
$$

Where $d_{i}(x)=\left(\underline{x}-\underline{\mu_{i}}\right)^{t}\left[\phi_{i} \Lambda_{i}^{-1} \phi^{T}\right]\left(\underline{x}-\underline{\mu_{i}}\right)=\underline{y_{i}^{t}} \Lambda_{i}^{-1} \underline{y_{i}}, \phi_{i}$ is the matrix that contains the eigenvectors of $\Sigma_{i}, \Lambda_{i}$ is the diagonal matrix that contains the eigenvalues of $\Sigma_{i}$, and $\underline{y_{i}}=\phi_{i}^{t}\left(\underline{x}-\underline{\mu_{i}}\right)$ is the weight vector obtained by projecting $\underline{x}$ onto sub-class $\underline{w}_{i}$.

The Mahalanobis Distance can be written as

$$
d_{i}(x)=\sum_{j=1}^{N} \frac{y_{i j}^{2}}{\lambda_{i j}}
$$

We use the K eigenvectors for projection process, and then we can write the expression of Mahalanobis Distance in (3) by the following:

$$
\begin{gathered}
\hat{d}_{i}(x)=\sum_{j=1}^{K} \frac{y_{i j}^{2}}{\lambda_{i j}}+\frac{1}{\rho_{i}} \sum_{j=K+1}^{N} y_{i j}^{2} \\
\hat{d}_{i}(x)=\sum_{j=1}^{K} \frac{y_{i j}^{2}}{\lambda_{i j}}+\frac{1}{\rho_{i}} \varepsilon_{i}^{2}(\underline{x})
\end{gathered}
$$

The first term is corresponding to the projection $\mathrm{x}$ onto k-dimensional principle subspace of sub-class $w_{i}$, while the second term is corresponding to the reconstruction error in $\underline{x}$ with respect to subspace of sub-class $w_{i}$. let $\hat{x}$ the approximate of $\underline{x}$ after reconstruction using $\mathrm{k}$ eigenvectors, then we can define the reconstruction error by:

$$
\begin{aligned}
& \varepsilon_{i}^{2}(\underline{x})=\|\underline{x}-\hat{x}\|^{2}=\left\|\underline{x}-\left(\mu_{i}+\sum_{j=1}^{K} y_{i j} \phi_{i j}\right)\right\|^{2} \\
& \varepsilon_{i}^{2}(\underline{x})=\left\|\left(\underline{x}-\mu_{i}\right)-\sum_{j=1}^{N} y_{i j} \phi_{i j}-\sum_{j=K+1}^{N} y_{i j} \phi_{i j}\right\|^{2}
\end{aligned}
$$




$$
\varepsilon_{i}^{2}(\underline{x})==\left\|\sum_{j=K+1}^{N} y_{i j} \phi_{i j}\right\|^{2}=\sum_{j=K+1}^{N} y_{i j}^{2}
$$

from (2) and (4) we can compute the estimate density as the following:

$$
\begin{gathered}
\hat{p}\left(\underline{x} \mid w_{i}\right)=\left[\frac{\left.\exp \left(-\frac{1}{2} \sum_{j=1}^{K} \frac{y_{i j}^{2}}{\lambda_{i j}}\right)\right]\left[\frac{\exp \left(\frac{-\varepsilon_{i}^{2}(\underline{x})}{2 \rho_{i}}\right)}{(2 \pi)^{\frac{K}{2}} \prod_{j=1}^{K} \lambda_{i j}}\right]}{\left(2 \pi \rho_{i}\right)^{\frac{N-K}{2}}}\right] \\
\hat{p}\left(\underline{x} \mid w_{i}\right)=\hat{p}_{t}\left(\underline{x} \mid w_{i}\right) \cdot \hat{p}_{e}\left(\underline{x} \mid w_{i}\right)
\end{gathered}
$$

The first term is the true marginal density in the subspace of sub-class $w_{i}$, and the second term is the estimated marginal density in the subspace that is orthogonal to subspace of subclass $w_{i}$.

The Optimal value of $\rho_{i}$ is determined by minimizing the cost function $J(\rho)$ which defined by relative entropy between the true density $p\left(\underline{x} \mid w_{i}\right)$ and its estimate $\hat{p}\left(\underline{x} \mid w_{i}\right)[118]$ :

$$
\begin{gathered}
J(p)=E\left[\log \frac{p\left(\underline{x} \mid w_{i}\right)}{\hat{p}\left(\underline{x} \mid w_{i}\right)}\right] \\
J(p)=\frac{1}{2} \sum_{j=K+1}^{N}\left(\frac{\lambda_{i j}}{\rho_{i}}-1+\log \frac{\rho_{i}}{\lambda_{i j}}\right)
\end{gathered}
$$

By taking the derivative of $J$ with respect to $\rho_{i}$ and set it to zero,

$$
\rho_{\text {iopt }}=\frac{1}{N-K} \sum_{j=K+1}^{N} \lambda_{i j}
$$

The reconstruction error is corresponded to estimated marginal density in the subspace that is orthogonal to subspace of sub-class $w_{i}$. As a result, we propose that the evaluator assigns the sub-class $w_{i}$ to segment outcome $\underline{x}$ based on estimated marginal densities of segmentation outcome sub-classes and their priori probabilities:

$g_{i e}(\underline{\hat{x}}) \geq g_{j_{e}}(\underline{\hat{x}})$ for all $j \neq i$, where $g_{i e}(\underline{\hat{x}})$ is the discriminant function [117] . In other words, we assign $\underline{x}$ to sub-class $w_{i}$ if 


$$
i=\arg \left(\operatorname { m a x } _ { < j > } \left(-\frac{N-K}{2} \ln \left(2 \pi \rho_{j}\right)+\frac{-\varepsilon_{j}^{2}(\underline{x})}{2 \rho_{j}}+\ln \left(p\left(\omega_{j}\right)\right)\right.\right.
$$

Special Case 1: when we choose the same number of Eigen subspace of each sub-class $w_{i}$, and $\rho_{1}=\rho_{2}=\cdots=\rho$ we can write (8) as

$$
i=\arg \left(\operatorname { m a x } _ { < j > } \left(+\frac{-\varepsilon_{j}^{2}(\underline{x})}{2 \rho}+\ln \left(p\left(\omega_{j}\right)\right)\right.\right.
$$

Special case 2: as mentioned in [109] when we choose the same number of Eigen subspace of each sub-class $w_{i}, \rho_{1}=\rho_{2}=\cdots=\rho$, and the prior probabilities are the same we can write (8) as:

$$
i=\arg \left(\min _{<j>} \varepsilon_{j}^{2}(\underline{x})\right)
$$

In other words, the discriminant function will be based only on the reconstruction error.

\subsection{Experimental Results for Dental Image Segmentation Evaluation}

We employed 100 bitewing images and 100 periapical images selected from large dental radiographic databases [115][116] in order to build the training sets of sub-classes presented in section 6.2.1. The selected dental radiographs have different quality, different intensity contrast, and part of them contains dental fillings. We first segmented the selected dental images using the approach presented in the previous chapter, and then we classified the segmentation outcomes into seven sub-classes for the bitewing images, four sub-classes for upper jaw periapical images, and four sub-classes for lower jaw periapical images. These sub-classes presented in Table 9. In order to increase the number of segmentation outcomes that are corresponding to improper objects, we change the value of thresholding presented in section 5.2.2 up and down to obtain more undersegmentation, oversegmentation, background outcomes.

We build the training sets of each sub-class using the segmentation outcome of the 100 bitewing images, 50 upper periapical images, and 50 lower periapical images. We tried to avoid any biasing in the training sets, therefore, we choose teeth from different types (Canine, Incisor, Premolar, and Molar), different intensity, different positions, and with and 
without dental fillings. Each training set contains 100 objects that belong to the corresponded sub-class. We compute the Eigen subspace for each of the sub-classes presented in Table 9 as shown in section 6.4.

In order to evaluate the performance of the described approaches using a large database, we used three test sets selected from [115][116]. The first test set contains 800 bitewing images, the second test set contains 321 upper periapical images, and the third one contains 333 lower periapical images. All of the images used to build the test sets are different from the one used in the training sets, and they belong to different records. Each record contains different number of dental images with variable views. In addition, the dental images in the records have different qualities, different intensity contrasts, and part of them have dental fillings. We applied the segmentation approach presented in the previous chapter on the three test sets.

Table 10, Table 11, and Table 12 show the number of each subclass resulted from dental image segmentation for bitewing, upper jaw periapical, and lower jaw periapical images respectively. It is clear that the percentages of improper segments are $12.25 \%, 20.63 \%$, and $20.18 \%$ for bitewing, upper jaw periapical, and lower jaw periapical images respectively. Therefore, decreasing this percentage will enhance ADIS performance and the segmentation results.

In practice, the number of principle components used for projection and reconstruction process will affect the performance of the Eigen-space method. Therefore, we need to determine the optimal number of the principle component that is corresponded to lowest error rate in evolution for proper and improper teeth segments. We perform the evaluation using different number of principle components, and then we report the results of each one. Figure 83, Figure 84, and Figure 85 show a plot of error rate vs. the number of principal components for bitewing, upper periapical, and lower periapical image segmentation outcomes respectively. The optimal number of principle components for proper segments in bitewing, upper periapical, and lower periapical is 20,25, and 25 respectively. On the other hand, the optimal number of principle components for improper segments in bitewing, upper periapical, and lower periapical is 30,30 , and 25 respectively.

Table 13, Table 14, and Table 15 show the number of segments for each subclass resulted from dental image segmentation after automated evaluation for bitewing, upper periapical, 
and lower periapical respectively. These numbers correspond to optimal number of principle components shown in Figure 83, Figure 84, and Figure 85.

Table 10: The number of each subclass resulted from dental image segmentation for bitewing dental images.

\begin{tabular}{|c|c|c|c|}
\hline Subclass & $\begin{array}{c}\text { Number of Outcomes } \\
\text { for Bitewing set }\end{array}$ & $\begin{array}{c}\text { Total Number of } \\
\text { proper segments }\end{array}$ & $\begin{array}{c}\text { Total Number of } \\
\text { improper segments }\end{array}$ \\
\hline $\begin{array}{c}\text { Upper Jaw } \\
\text { Tooth }\end{array}$ & 2127 & 3876 & \\
\hline $\begin{array}{c}\text { Lower Jaw } \\
\text { Tooth }\end{array}$ & 1749 & & \\
\hline $\begin{array}{c}\text { Upper Jaw } \\
\text { Oversegmentation }\end{array}$ & 45 & \\
\hline $\begin{array}{c}\text { Upper Jaw } \\
\text { Oversegmentation }\end{array}$ & 74 & \\
\hline $\begin{array}{c}\text { Upper Jaw } \\
\text { Undersegmentation }\end{array}$ & 155 & \\
\hline $\begin{array}{c}\text { Upper Jaw } \\
\text { Undersegmentation }\end{array}$ & 243 & \\
\hline Background & 24 & \\
\hline
\end{tabular}

Table 11: The number of each subclass resulted from dental image segmentation for upper jaw periapical images.

\begin{tabular}{|c|c|c|c|}
\hline Subclass & $\begin{array}{c}\text { Number of Outcomes } \\
\text { for Bitewing set }\end{array}$ & $\begin{array}{c}\text { Total Number of } \\
\text { proper segments }\end{array}$ & $\begin{array}{c}\text { Total Number of } \\
\text { improper segments }\end{array}$ \\
\hline $\begin{array}{c}\text { Upper Jaw } \\
\text { Tooth }\end{array}$ & 746 & 746 & \\
\hline $\begin{array}{c}\text { Upper Jaw } \\
\text { Oversegmentation }\end{array}$ & 45 & & 194 \\
\hline $\begin{array}{c}\text { Upper Jaw } \\
\text { Undersegmentation }\end{array}$ & 93 & & \\
\hline Background & 56 & & \\
\hline
\end{tabular}


Table 12: The number of each subclass resulted from dental image segmentation for lower jaw periapical images.

\begin{tabular}{|c|c|c|c|}
\hline Subclass & $\begin{array}{c}\text { Number of Outcomes } \\
\text { for Bitewing set }\end{array}$ & $\begin{array}{c}\text { Total Number of } \\
\text { proper segments }\end{array}$ & $\begin{array}{c}\text { Total Number of } \\
\text { improper segments }\end{array}$ \\
\hline $\begin{array}{c}\text { Lower Jaw } \\
\text { Tooth }\end{array}$ & 788 & 788 & \\
\hline $\begin{array}{c}\text { Lower Jaw } \\
\text { Oversegmentation }\end{array}$ & 43 & & 199 \\
\hline $\begin{array}{c}\text { Lower Jaw } \\
\text { Undersegmentation }\end{array}$ & 105 & & \\
\hline Background & 51 & & \\
\hline
\end{tabular}

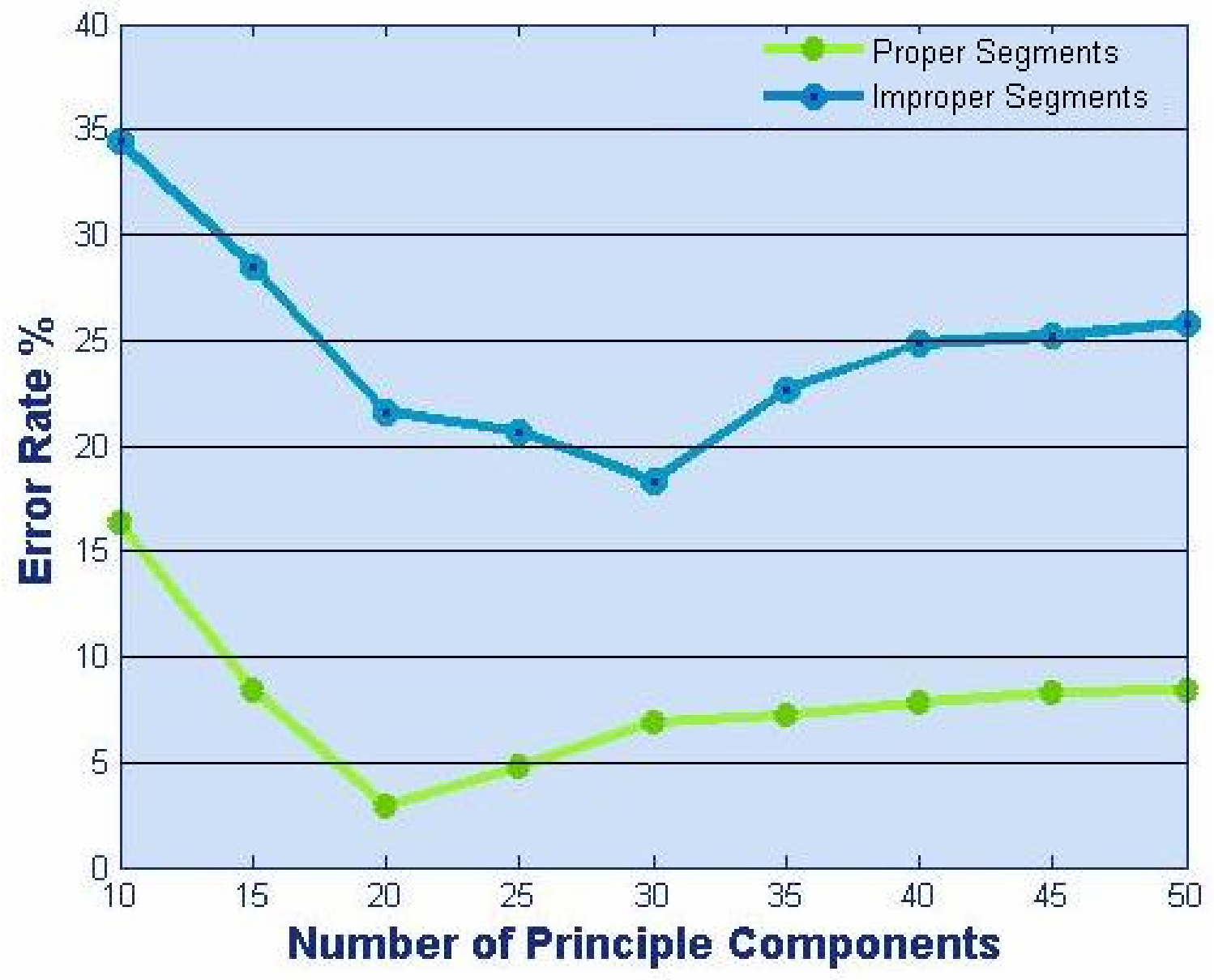

Figure 83: Variation in ASE performance of the Eigen-space method using different number of principal components for bitewing image segmentation outcomes. 


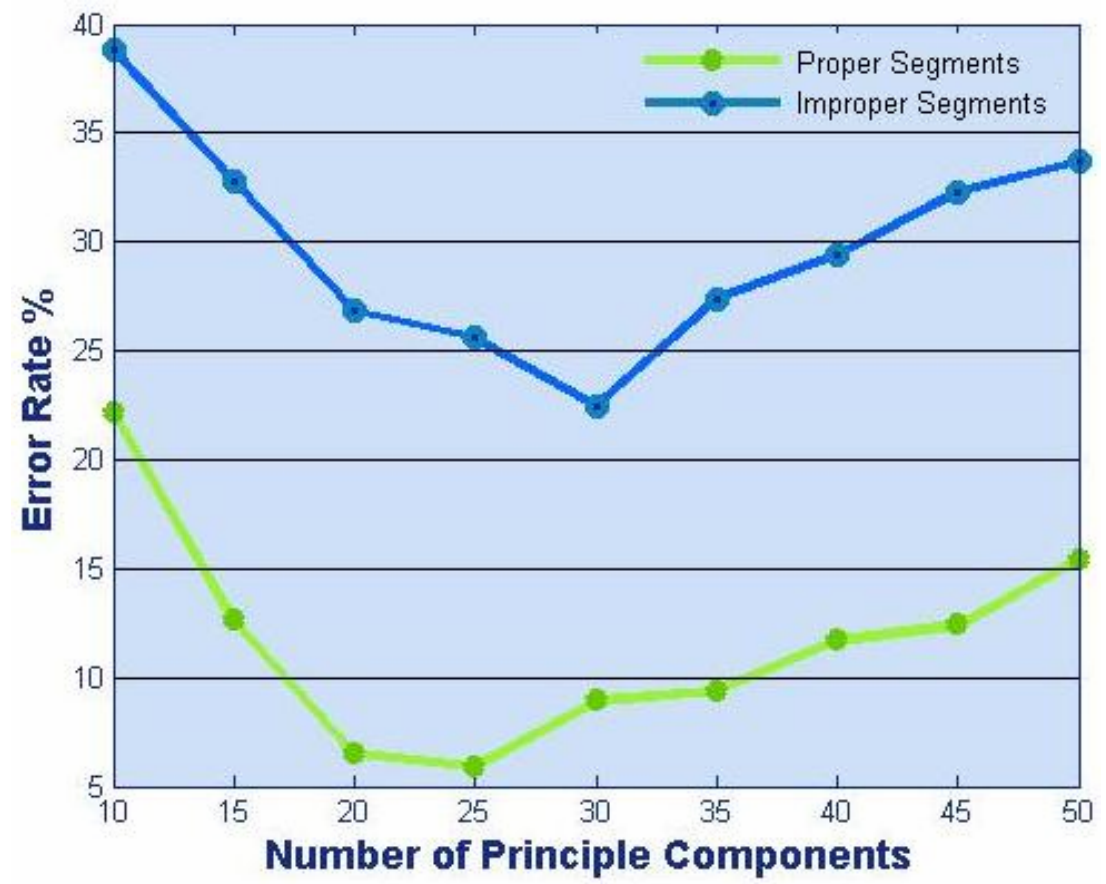

Figure 84: Variation in ASE performance of the Eigen-space method using different number of principal components for upper periapical image segmentation outcomes.

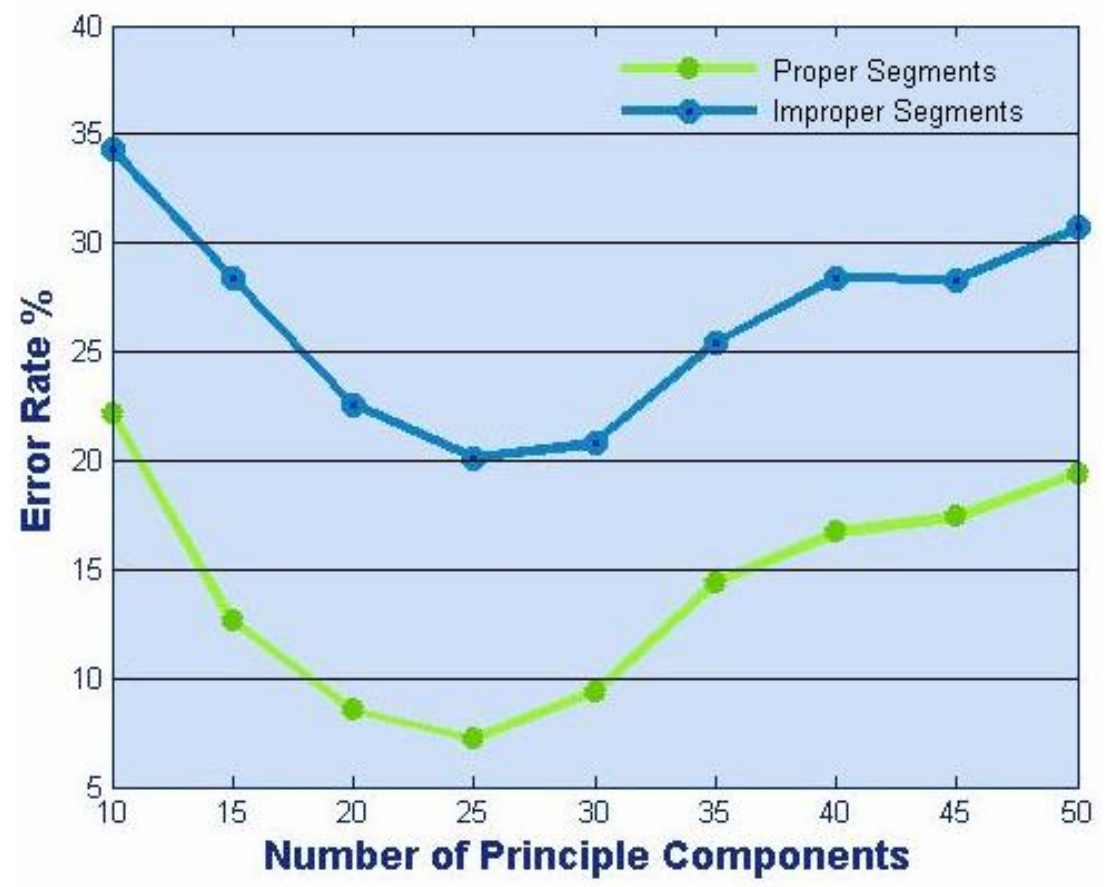

Figure 85: Variation in ASE performance of the Eigen-space method using different number of principal components for lower periapical image segmentation outcomes. 
Table 13: The number of each subclass resulted from dental image segmentation for bitewing dental images after evaluation.

\begin{tabular}{|c|c|c|c|}
\hline Subclass & $\begin{array}{c}\text { Number of Outcomes } \\
\text { for Bitewing set }\end{array}$ & $\begin{array}{c}\text { Total Number of } \\
\text { proper segments }\end{array}$ & $\begin{array}{c}\text { Total Number of } \\
\text { improper segments }\end{array}$ \\
\hline $\begin{array}{c}\text { Upper Jaw } \\
\text { Tooth }\end{array}$ & 2080 & 3765 & \\
\hline $\begin{array}{c}\text { Lower Jaw } \\
\text { Tooth }\end{array}$ & 1685 & & \\
\hline $\begin{array}{c}\text { Upper Jaw } \\
\text { Oversegmentation }\end{array}$ & 19 & & \multirow{2}{*}{118} \\
\hline $\begin{array}{c}\text { lower Jaw } \\
\text { Oversegmentation }\end{array}$ & 24 & & \\
\hline $\begin{array}{c}\text { Upper Jaw } \\
\text { Undersegmentation }\end{array}$ & 32 & \\
\hline $\begin{array}{c}\text { Upper Jaw } \\
\text { Undersegmentation }\end{array}$ & 37 & \\
\hline Background & 6 & \\
\hline
\end{tabular}

Table 14: The number of each subclass resulted from dental image segmentation for upper jaw periapical images after evaluation.

\begin{tabular}{|c|c|c|c|}
\hline Subclass & $\begin{array}{c}\text { Number of Outcomes } \\
\text { for Bitewing set }\end{array}$ & $\begin{array}{c}\text { Total Number of } \\
\text { proper segments }\end{array}$ & $\begin{array}{c}\text { Total Number of } \\
\text { improper segments }\end{array}$ \\
\hline $\begin{array}{c}\text { Upper Jaw } \\
\text { Tooth }\end{array}$ & 697 & 697 & \\
\hline $\begin{array}{c}\text { Upper Jaw } \\
\text { Oversegmentation }\end{array}$ & 20 & & 52 \\
\hline $\begin{array}{c}\text { Upper Jaw } \\
\text { Undersegmentation }\end{array}$ & 19 & & \\
\hline Background & 13 & & \\
\hline
\end{tabular}

Table 15: The number of each subclass resulted from dental image segmentation for lower jaw periapical images after evaluation.

\begin{tabular}{|c|c|c|c|}
\hline Subclass & $\begin{array}{c}\text { Number of Outcomes } \\
\text { for Bitewing set }\end{array}$ & $\begin{array}{c}\text { Total Number of } \\
\text { proper segments }\end{array}$ & $\begin{array}{c}\text { Total Number of } \\
\text { improper segments }\end{array}$ \\
\hline $\begin{array}{c}\text { Lower Jaw } \\
\text { Tooth }\end{array}$ & 720 & 720 & \\
\hline $\begin{array}{c}\text { Lower Jaw } \\
\text { Oversegmentation }\end{array}$ & 17 & & 4 \\
\hline $\begin{array}{c}\text { Lower Jaw } \\
\text { Undersegmentation }\end{array}$ & 14 & & \\
\hline Background & 14 & & \\
\hline
\end{tabular}


In Figure 86, we show examples where ASE is correctly evaluated the segmentation outcomes of different types of dental views. In Figure 87, we show examples where ASE where unable to evaluate the segmentation outcomes correctly. Figure 88 shows the confusion matrixes of ASE performance for various views of dental images .
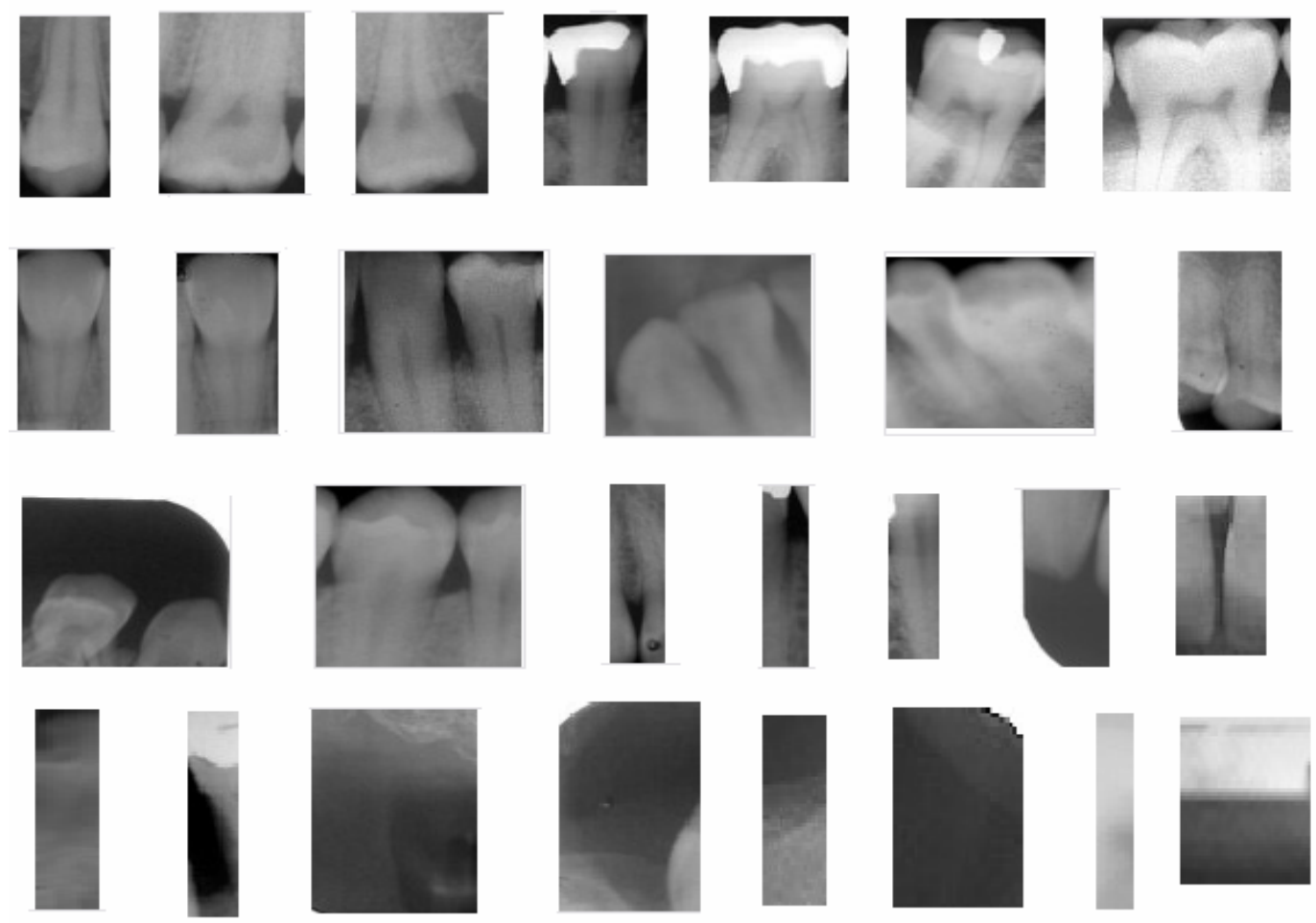

Figure 86: Examples of correctly evaluated segments.
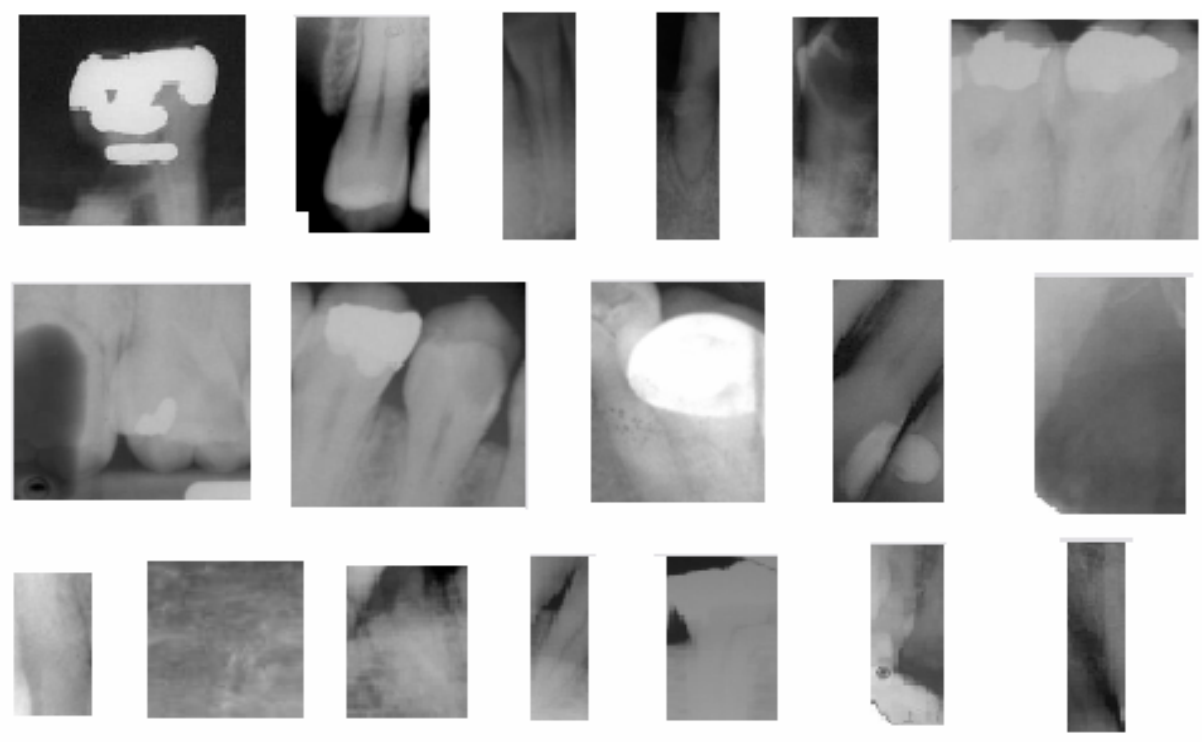

Figure 87: Examples of incorrectly evaluated segments 
Actual Outcome

\begin{tabular}{|c|c|c|c|}
\cline { 2 - 4 } & Bitewing & $\begin{array}{c}\text { Proper } \\
\text { segments }\end{array}$ & $\begin{array}{c}\text { Improper } \\
\text { Segments }\end{array}$ \\
\cline { 2 - 4 } Prediction & $\begin{array}{c}\text { Proper } \\
\text { segments }\end{array}$ & $97.2 \%$ & $2.8 \%$ \\
\cline { 2 - 4 } Outcome & $\begin{array}{l}\text { Improper } \\
\text { Segments }\end{array}$ & $21.8 \%$ & $72.18 \%$ \\
\cline { 2 - 4 } & \multicolumn{2}{|c}{} \\
\hline
\end{tabular}

FAR $=21.8 \%$

$\mathrm{FRR}=2.8 \%$

Actual Outcome

\begin{tabular}{|c|c|c|c|}
\cline { 2 - 4 } & $\begin{array}{c}\text { Lower } \\
\text { Periapical }\end{array}$ & $\begin{array}{c}\text { Proper } \\
\text { segments }\end{array}$ & $\begin{array}{c}\text { Improper } \\
\text { Segments }\end{array}$ \\
\cline { 2 - 4 } Prediction & $\begin{array}{c}\text { Proper } \\
\text { Segments }\end{array}$ & $91.3 \%$ & $8.6 \%$ \\
\cline { 2 - 4 } Outcome & $\begin{array}{c}\text { Improper } \\
\text { Segments }\end{array}$ & $22.6 \%$ & $77.3 \%$ \\
\hline
\end{tabular}

FAR $=22.6 \%$

$\mathrm{FRR}=8.6 \%$
Actual Outcome

\begin{tabular}{|c|c|c|c|}
\hline \multirow{3}{*}{$\begin{array}{l}\text { Prediction } \\
\text { Outcome }\end{array}$} & $\begin{array}{c}\text { Upper } \\
\text { Periapical }\end{array}$ & $\begin{array}{c}\text { Proper } \\
\text { segments }\end{array}$ & $\begin{array}{l}\text { Improper } \\
\text { Segments }\end{array}$ \\
\hline & $\begin{array}{c}\text { Proper } \\
\text { segments }\end{array}$ & $93.4 \%$ & $6.5 \%$ \\
\hline & $\begin{array}{l}\text { Improper } \\
\text { Segments }\end{array}$ & $26.8 \%$ & $73.2 \%$ \\
\hline
\end{tabular}

FAR $=26.8 \%$

$\mathrm{FRR}=6.5 \%$

Actual Outcome

\begin{tabular}{|l|c|c|c|}
\cline { 2 - 4 } & All Views & $\begin{array}{c}\text { Proper } \\
\text { segments }\end{array}$ & $\begin{array}{c}\text { Improper } \\
\text { Segments }\end{array}$ \\
\cline { 2 - 4 } $\begin{array}{l}\text { Prediction } \\
\text { Outcome }\end{array}$ & $\begin{array}{c}\text { Proper } \\
\text { segments }\end{array}$ & $95.7 \%$ & $4.2 \%$ \\
\cline { 2 - 4 } & $\begin{array}{c}\text { Improper } \\
\text { Segments }\end{array}$ & $23 \%$ & $77 \%$ \\
\cline { 2 - 4 }
\end{tabular}

FAR $=23 \%$ FRR $=4.2 \%$

Figure 88: Confusion matrixes for various views of dental images.

We can observe the following from the previous figures and tables in this section:

1- Overall, the percentage of improper segment was $15.5 \%$ before evaluation and $4.3 \%$ after the evaluation. In addition, the percentage of proper segments that evaluated as improper segments is $4.2 \%$, while, the percentage of improper segments that evaluated as proper segments is $23.1 \%$. the overall error of evaluation is $7.6 \%$

2- In the bitewing images, the percentage of improper segment was $12.25 \%$ before evaluation and 3\% after the evaluation. The percentage of proper segments that evaluated as improper segments is $2.8 \%$, while, the percentage of improper segments that evaluated as proper segments is $21.8 \%$. the overall error of evaluation is $4 \%$

3 - In the upper periapical images, the percentage of improper segment was $20.6 \%$ before evaluation and $6.9 \%$ after the evaluation. The percentage of proper segments that evaluated as improper segments is $6.5 \%$, while the 
percentage of improper segments that evaluated as proper segments is $26.8 \%$. the overall error of evaluation is $10.7 \%$

4- In the lower periapical images, the percentage of improper segment was $20.18 \%$ before evaluation and $5.88 \%$ after the evaluation. The percentage of proper segments that evaluated as improper segments is $8.6 \%$, while, the percentage of improper segments that evaluated as proper segments is $22.6 \%$. The overall error of evaluation is $11.35 \%$.

5- The evaluation of bitewing dental image segmentation outcomes is more accurate than the ones in the periapical views. The main reasons of that because the periapical images have more information about the teeth and the variations in the root shape. In addition, the high intensity of the bones in the periapical images increases the errors.

6- The performance of evaluator in detecting the undersegmentation and oversegmentation outcomes in bitewing images is superior to its performance in both views of periapical images.

7- The performance of evaluator in detecting the background outcomes in bitewing images and the periapical views is very close.

8- The error rate in detecting oversegmentation and undersegmentation outcomes in the upper periapical is higher than the one in the lower periapical. This is due to presence of teeth that have three roots in the upper jaw which increase the interference between the teeth.

In order to study the impact of ASE on performance of the matching process, we used the proposed ADIS system developed by WVU research team. The matching process and decision making scheme introduced in [110]. We used closed set identification database that contains 24 dental records belongs to 12 subjects. Twelve dental records are AM records and twelve are PM records. We run each AM record against all PM records to find its match with and without the presence of ASE. The matching result of each PM record belongs to matched, unmatched, or undetermined list. Table 16 shows the results of matching with/without ASE using ADIS. The results show that ASE increases the efficiency of ADIS by increasing the number of match cases and decreasing the number of undetermined cases. Two of unmatched cases presented because there is no corresponding tooth to tooth between 
$\mathrm{AM}$ record and PM record to implement matching. However, the ASE eliminates the improper segments results from errors in cropping and film type classification.

Table 16: Result of Matching PM records against AM records for 12 Subject using ADIS with/without ASE

\begin{tabular}{|c|c|c|c|}
\cline { 2 - 4 } \multicolumn{1}{c|}{} & \# of Matched Cases & $\begin{array}{c}\text { \# of Undetermined } \\
\text { Cases }\end{array}$ & $\begin{array}{c}\text { \# of Unmatched } \\
\text { Cases }\end{array}$ \\
\hline $\begin{array}{c}\text { Matching Results } \\
\text { without ASE }\end{array}$ & 1 & 9 & 2 \\
\hline $\begin{array}{c}\text { Matching Results } \\
\text { with ASE }\end{array}$ & 7 & 2 & 3 \\
\hline
\end{tabular}

\subsection{Experimental Results for Ear Image Segmentation}

\section{Evaluation}

We build the training set of sub-classes presented in section 6.2.2 for ear image segmentation evaluation using 30 subjects of database [114]. In the selecting criteria, we tried to avoid any biasing in the training sets; therefore, we take into consideration choosing different genders, races, views, background, height, and illumination.

We segmented the facial images of selected subjects using the approach presented in the previous chapter, and then we classify segmentation outcomes into five sub-classes as mentioned in section 6.2.2. We increase the number of segmentation outcomes that are corresponding to improper objects by changing the value of thresholding up and down. Each training set contains 200 objects that belong to the corresponded sub-class. After that, we compute the Eigen-space for each sub-class in the training sets.

To evaluate the performance of ear segmentation evaluation, we build four test sets selected from databases [114] for 190 subjects respectively. We applied the segmentation approach presented in the previous chapter on the facial images of selected subjects. Each test set of the proper segments contains 2000 ear images with different genders, races, views, height, and illumination. For each test set that belongs to partially succeeded or improper segments, we use 1000 segments.

In order to determine the optimal number of the principle component that is corresponded to lowest error rate in evolution, we use various number of principle components for projection and reconstruction process. 
Table 17 show the error rate vs. the number of principal components for correct ear segments and incorrect ear segments. It also shows that the minimum percentage of error in correctly recognizing the non-ear segments is $3.5 \%$.

Table 17: Error rates vs. of number of principle components for ear and non ear segments

\begin{tabular}{|c|c|c|}
\hline $\begin{array}{c}\text { Number of } \\
\text { Principle } \\
\text { components }\end{array}$ & $\begin{array}{c}\text { Percentage of } \\
\text { error for ear } \\
\text { segments }\end{array}$ & $\begin{array}{c}\text { Percentage of } \\
\text { error for non-ear } \\
\text { segments }\end{array}$ \\
\hline 10 & 12.25 & 18.4 \\
\hline 15,20 & 9.8 & 14.7 \\
\hline 25 & 7.45 & 12.8 \\
\hline 30,35 & 5.3 & 8.4 \\
\hline 40 & 3.3 & 5.4 \\
\hline 45 & 2.3 & 4.2 \\
\hline 50,55 & 2.3 & 3.5 \\
\hline 60 & 3.85 & 5.3 \\
\hline
\end{tabular}

In Figure 89, we show examples of successful ear segmentation outcomes evaluation of different facial image views. In Figure 90, we show examples of incorrectly ear segmentation outcomes evaluation of facial images.
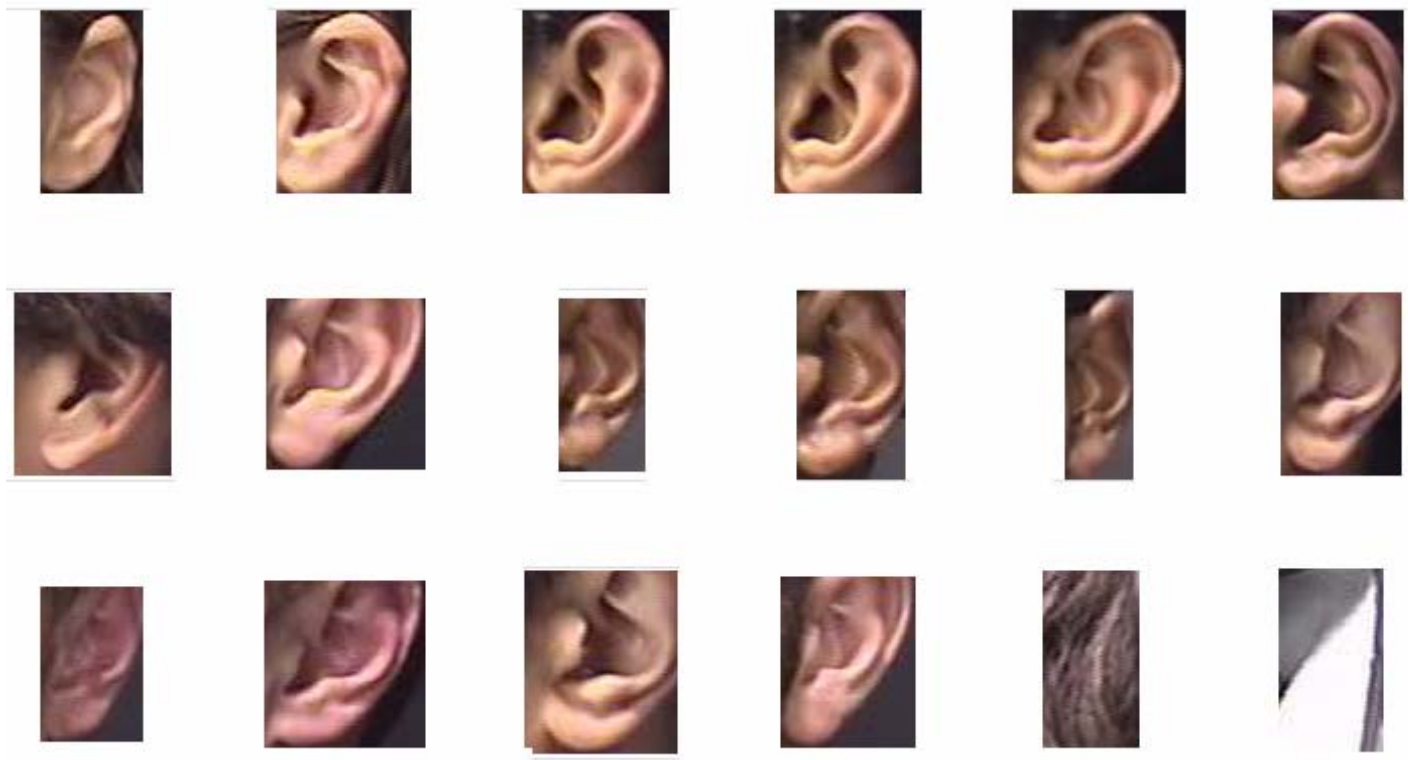

Figure 89: Examples of correctly evaluated segments. 

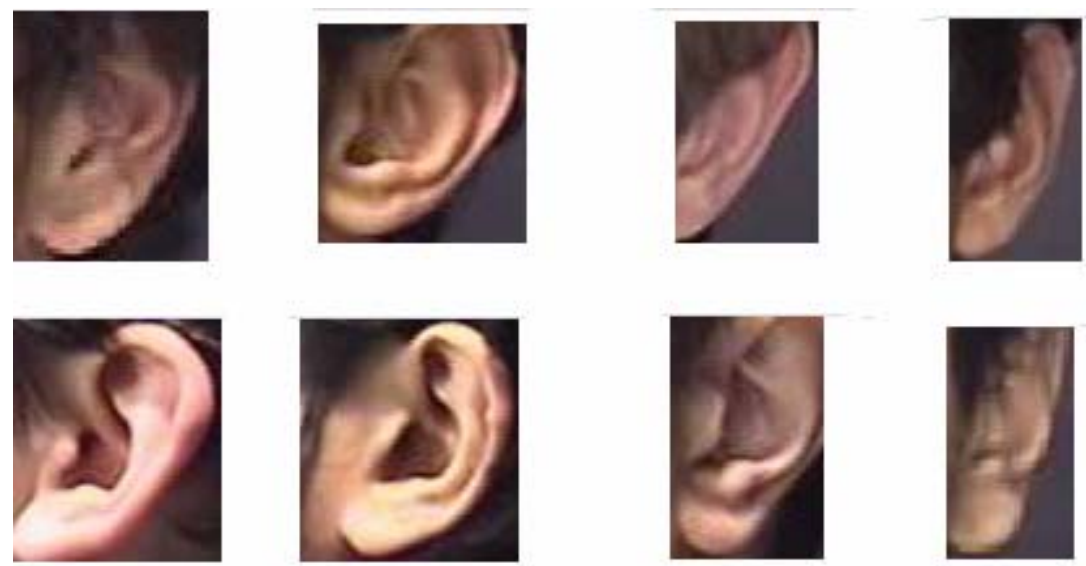

Figure 90: Examples of incorrectly evaluated segments.

In order to study the impact of evaluation on the performance of the matching process, we used standard Eigen-space recognition system. The process of normalization and projection of matcher are the same as the one introduced in sections 6.3.26.4. We used close set identification database that contains 100 subjects chosen from [113]. All ear images in the database have been segmented from facial images that have 15 degree angel view. For verification process, we used 1000 ear images belonging to the same 100 subjects in the database with angel views between $[0,30]$. We chose 10 ear segments for each subject, seven of them are proper segments, and three of them are improper.

We run three sets of images into the matcher. The first set contains the 700 proper segments, the second set contains 1000 proper and improper segments without using ASE, and the third set contains the proper and improper segments with presence of ASE. The ROC curve of running the three sets is shown in Figure 91. The results show that the performance of the matcher after including ASE is comparable to its performance when the segmentation outcomes are ideal. In addition, we save time by eliminating the improper segments to be processed. 


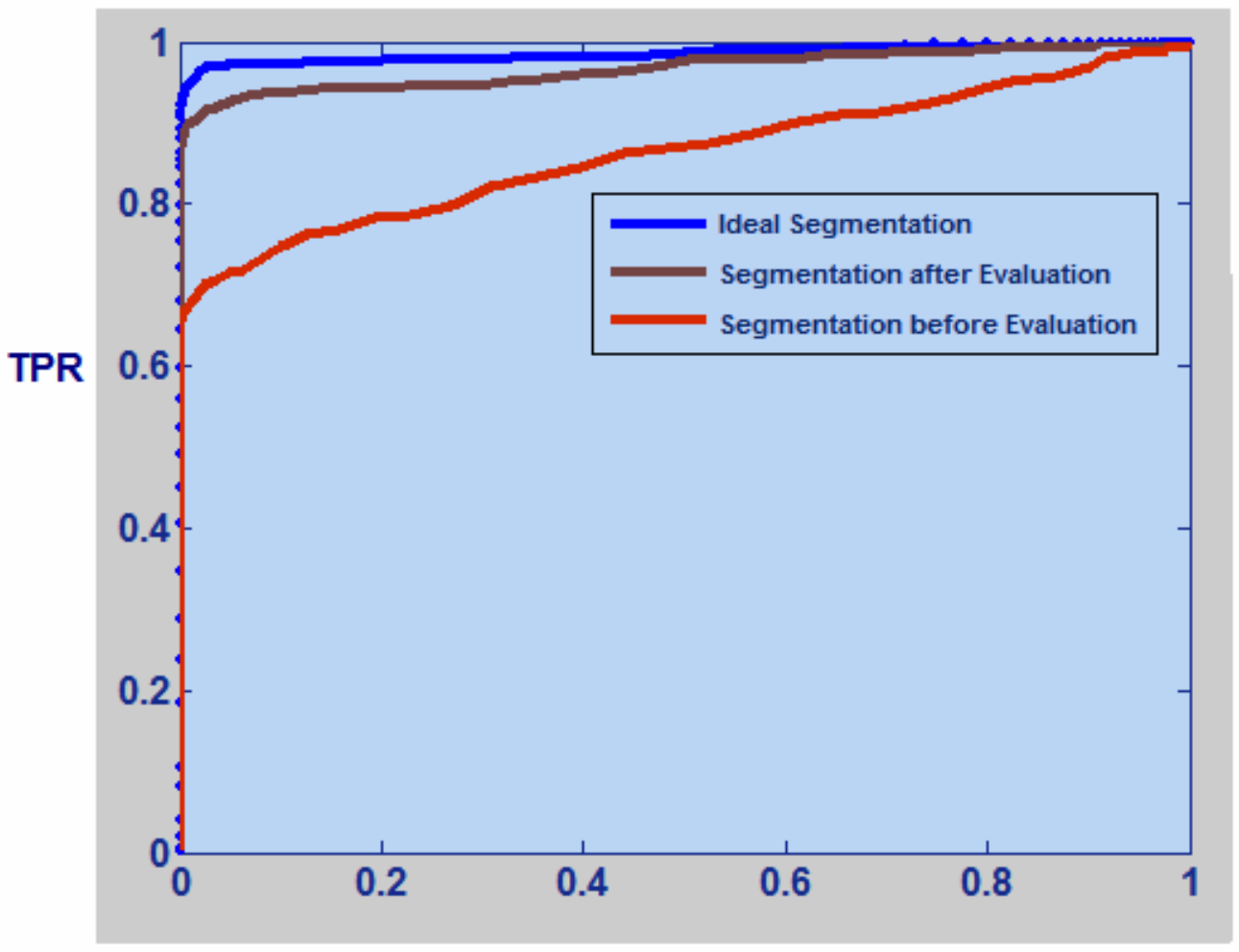

\section{FPR}

Figure 91: ROC Curve of matcher performance using Ideal segmentation, segmentation without ASE, and segmentation with presence of ASE.

\subsection{Summary}

In this chapter we presented a fully real time ASE used in the biometric systems to increase the performance efficiency, and to reduce the wasting time. We handle two different types of biometric image segmentation outcomes, the dental radiograph image segmentation outcomes evaluation, and ear segmentation outcomes evaluation from facial images. Our approach is based on low computational-cost and appearance-based features. It consists of two stages, off-line and on-line. In the off-line stage, we initially classify the segmentation outcomes in the biometric system into main sub-classes and create training sets, and then we standardize the view of the segmentation outcome. Finally, we create the Eigen-spaces corresponding to the chosen training sets. In the on-line stage, we project the segmentation outcome onto the Eigen-spaces after normalization, and then we used the evaluation scheme in order to determine if the outcome is proper or improper segment. 
In the evaluation of dental image segmentation outcomes, we used three tests of dental radiograph images for bitewing, upper periapical, and lower periapical views. The total number of test images is 1454 taken from 216 dental records. The results show that we reduce the percentage of improper outcomes produced from segmentation from $15.5 \%$ before evaluation to $4.1 \%$ after evaluation. In addition, the results show that the performance of evaluation of bitewing image segmentation outcome is better than its performance in the periapical views. This is due to the presence of jaw bones and teeth root which increase the complexity of the problem. The performance of evaluator in detecting the oversegmentation and undersegmentation outcomes in the bitewing views is better than the one in the periapical views, however; its performance in detecting the background outcomes can be considered the same.

The results also showed that the evaluator is more sensitive to detect the undersegmentation outcomes rather than the oversegmentation outcomes. This is due to presence of three types of teeth in terms of the number of the roots. Additionally, the presence of the filling that spread on more than tooth will increase the difficulties.

Although the evaluator will eliminate some of the proper teeth, most of these proper segments have poor quality because of very low intensity, fillings, or teeth overlapping. However; the percentage of lost qualified teeth can be neglected comparing to percentage of eliminating the improper outcomes.

In the evaluation of ear image segmentation outcomes, we classify the outcomes of segmentation outcomes into five sub-classes, two of them correspond to correct ear segments, two correspond to partially ear segments, and one corresponds to non ear objects. The results show that the percentage of error in detecting the non-ear segments is $3.5 \%$, while it is $2.3 \%$ in detecting the ear segments. In addition, the performance of the matcher after including ASE is comparable to its performance when the segmentation outcomes are ideal 


\section{Chapter 7: Conclusion}

In this dissertation, we addressed the problem of fully automated image segmentation in the context of dental and ear biometrics. We also address the problem of evaluating the quality of segmented image by designing an ASE.

Image segmentation is essential for guiding the process of identification process. We employ the mathematical morphology to segment teeth and ear from dental radiographs and facial images. We use mathematical morphology operator to highlight the desired objects and suppress the others, and then threshold the resulting image to separate the desired objects from the background. We next analyze the connected components obtained from the thresholded image based on their geometric properties in order to isolate the desired objects. Results on dental radiograph images show that our approach has the highest optimality and lowest failure rate compared to the other automated approaches. In addition, it can deal with bitewing and periapical dental images.

In ear images, the experimental results show that our segmentation approach achieves more than $90 \%$ accuracy based on three different sets of 3750 facial images for 376 persons, and it can deal with different poses, backgrounds, and illumination.

We also present an approach for the automated evaluation of the quality of segmented images. Our approach is based on low computational-cost appearance-based features, and consists of two stages: off-line and on-line. In the off-line stage, we generate training sets by manually classifying the segmentation outcomes of proposed approach into several subclasses. Next, we create the Eigen-spaces corresponding to the different training sets. In the on-line stage, we project the outcome of segmentation onto the Eigen-spaces after view normalization, and use a Bayesian Classifier in order to determine whether the segmentation outcome is proper or improper segment.

Results on dental images show that using ASE reduced the percentage of improper segmentation outcomes from $15.5 \%$ (without using ASE) to $4.1 \%$. In addition, ASE enhanced the efficiency of ADIS and matching process [110] by increasing the number of matched cases and decreasing the number of undetermined cases generated from the matching process. This is because ASE eliminates the improper segments produced not only from errors in segmentation but also from errors in cropping and film type classification.

Results on ear images show that the percentage of error in detecting the non-ear segments is 
$3.5 \%$, while it is $2.3 \%$ in detecting the ear segments. In addition, the performance of the matcher after including ASE is comparable to its performance when the segmentation outcomes are ideal.

Our plan for the future is to expand the functionality of ADIS to work on oral photographs, dental Models, and CT scans. In addition we plan to develop algorithms to estimate the quality of dental images, and to predict dental clinical problems (e.g., bone density).

In the context of ear images, we plan to develop the functionality of ear segmentation to work with cluttered background. 


\section{Bibliography}

[1] A.K. Jain, L. Hong, and S. Pankanti, "Biometrics: Promising Frontiers for Emerging Identification Market”, Comm. ACM, pp. 91-98, Feb. 2000.

[2] P. Stimson \& C. Mertz, Forensic Dentistry. CRC Press, 1997.

[3] Gustafson \& Ghosta, Forensic Odontology. American Elsevier Pub. Co. 1996.

[4] A. Iannarelli, Ear Identification. Forensic Identification Series. Paramont Publishing Company, Fermont, CA, 19892

[5] S. White, and M. Pharoah, Oral Radiology Principles and Interpretation. Mosby, Inc. Fourth Edition 2000.

[6] G. Fahmy, D. Nassar, E. Haj-Said, H. Chen, O. Nomir, J. Zhou, R. Howell, H. H. Ammar, M. Abdel-Mottaleb and A. K. Jain, "Towards an Automated Dental Identification System (ADIS)", Proc. the International Conference on Biometric Authentication (ICBA), pp. 789796, Hong Kong, July 2004.

[7] R. Khanna., S. Weicheng "Automated Fingerprint Identification System (AFIS) Benchmarking Using the National Institute of Standards and Technology (NIST) Special Database 4" Security Technology, 1994. Proceedings Institute of Electrical and Electronics Engineers 28th Annual 1994 International Carnahan Conference on 12-14 Oct. 1994 pp. $188-194$.

[8] A. K Jain and H. Chen, "Matching of Dental X-ray Images for Human Identification", Pattern Recognition, vol. 37, pp. 1519-1532, 2004.

[9] O. Nomir and M. Abdel-Mottaleb, "A System for Human Identification from X-Ray Dental Radiographs”, Pattern Recognition, vol. 38, pp. 1295-1305, 2005.

[10] J. Zhou, and M. Abdel-Mottaleb, "A Content-based System for Human Identification Based on Bitewing Dental X-Ray Images", to appear in Pattern Recognition.

[11] Mohamed Abdel-Mottaleb and Jindan Zhou, "A System for Ear Biometrics from Face Profile Images.

[12] United States United States Army Institute of Dental Research Walter Reed Army Medical Center,Computer Assisted Post Mortem Identification via Dental and other CharacteristicsUSAIDR Information Bulletin. Vol. 5, No. 1, Autumn 1990.

[13] Dr. J. McGivney et al. WinID2 software available (www.winid.com.).

[14] L. Lorton , M. Rethman , and R. Friedman The CAPMI system: A Computer Based Identification Program. J Forensic Sci, 1988; Vol 33: pp.977 - 984. 
[15] J. McGivney. Commentary on: Lewis C. WinID2 veerses CAPMI4: Two ComputerAssisted Dental Identification Systems. J Forensic Sci 2002;47(3) pp. 536-538.

[16] C. Lewis, and L. Leventhal, Locator system versus WinID3 versus CAPM14: Identifying Vicimsfrom Dental Remains in a Large Disaster. J of Forensic Identification 2004; 52(4) pp. 185-202.

[17] CM. Bowers ,RJ. Johansen . Digital Imaging Methods As an Aid in Dental Identification of Human Remains. J Forensic Sci 2002;47(2): pp. 354-359.

[18] D. E. Nassar, A Prototype Automatic Dental Identification System (ADIS). Masters Thesis, Department of Electrical and Computer Engineering - WVU, April. 2001.

[19] A. Jain and A. Ross, "Biometric Recognition: Techniques, Applications and Challenges", Tutorial Presented at the International Conference on Pattern Recognition (ICPR), Cambridge, UK, August 2004.

[20] A. Jain, A. Ross and S. Prabhakar, " An Introduction to Biometric Recognition", IEEE Transactions on Circuits and Systems for Video Technology, Special Issue on Image- and Video-Based Biometrics, Vol. 14, No. 1, pp. 4-20, January 2004.

[21] A. Iannarelli, Ear Identification. Forensic Identification Series. Paramont Publishing Company, Fermont, CA, 1989.

[22] C. Kyong , K.W. Bowyer, S. Sarkar, and B. Victor; Comparison and Combination of Ear and Face Images in Appearance-based Biometrics Pattern Analysis and Machine Intelligence,IEEE Transactions on Volume 25, Issue 9, Sept. 2003 pp. 1160 - 1165.

[23] A. Hoogstrate, H. Van den Heuvel, E. Huyben, "Ear Identification Based on Surveillance Camera's Images", Version updated May $31,2000$.

http://www.forensicevidence.com/site/ID/IDearCamera.html

[24] M. Burge, and W. Burger, Ear Biometrics. In A. Jain R. Bolle and S. Pankanti, editors, BIOMETRICS: Personal Identification in a Networked Society, pp. 273-286. Kluwer Academic, 1998

[25] M. Burge, , and W. Burger,; Ear Biometrics in Computer Vision Pattern Recognition, 2000. Proceedings. 15th International Conference onVolume 2, 3-7 Sept 2000 pp. 822 - 826 Vol. 2.

[26] C. Kyong; K.W. Bowyer; S. Sarkar, and B. Victor; Comparison and Combination of Ear and Face Images in Appearance-based Biometrics Pattern Analysis and Machine Intelligence,IEEE Transactions on Vol. 25, Issue 9, Sept. 2003 pp. 1160 - 1165.

[27] H.J. Zhang, Z.C. Mu; W. Qu, L. M. Liu, and C. Y. Zhang; "A Novel Approach for Ear Recognition Based on ICA and RBF Network" Machine Learning and Cybernetics, 2005. Proceedings of 2005 International Conference on Volume 7, 18-21 Aug. 2005 pp. 4511 4515 Vol. 7. 
[28] D.J. Hurley, M.S. Nixon, , and J.N Carter; A New Force Field Transform for Ear and Face Recognition. In Proceedings of the IEEE 2000 International Conference on Image Processin ICIP 2000b, pp. 25-28.

[29] R. C. Gonzales and R. E. Woods, Digital Image Processing, Second Edition, Prentice Hall, 2002.

[30] P.K. Sahoo, S. Soltani, and A.K.C.Wong. A Survey of Thresholding Techniques. Comput. Vis. Graph. Im. Proc., 41: pp. 233-260, 1988.

[31] H. Suzuki and J. Toriwaki, "Automatic Segmentation of Head MRIimages by Knowledge Guided Thresholding," Computerized Med. Imag.Graphics, Vol. 15, no. 4, pp. 233-240, July-Aug. 1991.

[32] E. d. S. Filho, Y. Saijo, T. Yambe, A. Tanaka, and M. Yoshizawa: Segmentation of Calcification Regions in Intravascular Ultrasound Images by Adaptive Thresholding. CBMS 2006: pp. 446-454.

[33] M. Xu, W. K. Luk, P. D. Cutler, and W. M. Digby, "Local Threshold for Segmented Attenuation Correction of PET Imaging of the Thorax.," IEEE Trans Nucl Sci, Vol. 41, pp. $1532-1537,1994$.

[34] A. Castro, B. Arcay, C. Dafonte, A. Santos, and J. Suarez" Development of an Analysis System of the X-rays of Bones [for prosthesis placement]" Proceedings of the 22nd Annual International Conference of the IEEE Engineering in Medicine and Biology Society, 2000. Vol. 3, 23-28 July 2000 pp. 1795 - 1798 Vol. 3.

[35] K.V. Mardia, and T.J. Hainsworth , "A Spatial Thresholding Method for Image Segmentation", IEEE Transactions on Pattern Analysis and Machine Intelligence, Vol. 10, Issue 6, Nov. 1988 pp. $919-927$.

[36] D. L. Pham, C. Xu, and J. L. Prince, "A Survey of Current Methods in Medical Image Segmentation", in Annual Review of Biomedical Engineering, Vol. 2, eds. M. L. Yarmush, K. R. Diller, and M. Toner, Annual Reviews, pp.315-337, 2000.

[37] Medical Image Segmentation, Volume Representation and Registration Using Spheres in the Geometric Algebra Framework - ARTICLE Pattern Recognition, In Press, Corrected Proof, Available online 23 August 2006.

[38] R. Pohle, and K. D. Toennies, "A New Approach for Model-Based Adaptive Region Growing in Medical Image Analysis", Computer Analysis of Images and Patterns: 9th International Conference, CAIP 2001 Warsaw, Poland, September 5-7, 2001, Proceedings, Vol. 2124/2001, pp 238.

[39] R. Pohle, and K.D Toennies "Segmentation of Medical Images Using Adaptive Region Growing”, Proc. SPIE Vol. 4322, pp. 1337-1346, Medical Imaging 2001. 
[40] S. Gonzlez; R. Rojas, J.H. Sossa-Azuela, F. A. BARRIOS, and M.K. Hanson "MRI Segmentation Based on Region Growing with Robust Estimation" Proceedings of SPIE Vol. 3661 Medical Imaging 1999: Image Processing, Kenneth M. Hanson, Editor, May 1999 , pp. 880-885.

[41] Y. Li, D. Lu, X. Lu; and J. Liu "Interactive Color Image Segmentation by Region Growing Combined with Image Enhancement Based on Bezier Model", Proceedings. Third International Conference on Image and Graphics, 2004 18-20 Dec. 2004 pp. 96 - 99.

[42] S. Lee, MM. Crawford "Unsupervised Classification Using Spatial Region Growing Segmentation and Fuzzy Training", Proceedings. 2001 International Conference on Image Processing, 2001. Vol. 1, 7-10 Oct. 2001 pp. :770 - 773.

[43] J. Yen, and R. Langari Fuzzy Logic: Intellicenc e, Cont rol, and Information, Prentice Hall.

[44] Y. Zhao, M. Li , “ A Modified Fuzzy C-Means Algorithm for Segmentation of MRI” Computational Proceedings. Fifth International Conference onIntelligence and Multimedia Applications, 2003. ICCIMA 2003. pp. 391- 395 Sept. 2003.

[45] S. Shenli, W.A. Sandham, M. H. Granat, M. F. Dempsey, J. Patterson, Fuzzy Clustering Based Applications to Medical Image Segmentation" Proceedings of the 25" Annual Intemational Conference of the IEEE EMBS Cancun, Mexico September 17-21,2003.

[46] N. Archip, R. Rohling, P. Cooperberg, H. Tahmasebpour, and S. K. Warfield, "Spectral Clustering Algorithms for Ultrasound Image Segmentation" J. Duncan and G. Gerig (Eds.): MICCAI 2005, LNCS 3750, pp. 862-869, 2005. c Springer-Verlag Berlin Heidelberg 2005.

[47] Z. Yu; and H.S. Wong; , "Genetic-based K-means Algorithm for Selection of Feature Variables" 18th International Conference on Pattern Recognition, 2006. ICPR 2006. Vol. 2, 20-24 Aug. 2006 pp. $744-747$.

[48] Y. Feng,W. Chen,” Brain MR Image Segmentation Using Fuzzy Clustering with Spatial Constraints Based on Markov Random Field Theory" Springer Berlin / Heidelberg, Vol. 3150/2004 pp. 188-195.

[49] Y. Zhu, J. Yang, G. Geng, and Y. Zheng, "Adaptive Ribbon: a Hybrid Model for MR Cerebral Cortical Segmentation and Representation",submitted to Medical Image Analysis.

[50] L. Fausett, “ Fundamentals of Neural Networks, Prentice Hall.

[51] S.C.Amartur, D. Piraino, Y. Takefuji, "Optimization Neural Networks for the Segmentation of Magnetic Resonance Images" IEEE Transactions on Medical Imaging,Vol. 11, Issue 2, June 1992 pp. 215 - 220.

[52] K. S. Cheng; J. S. Lin; C.W. Mao, " The Application of Competitive Hopfield Neural Network to Medical Image Segmentation" IEEE Transactions on Medical Imaging, 
Vol. 15, Issue 4, Aug. 1996 pp. 560 - 567.

[53] Z. Dokur, M.N. Kurnaz, T. Olmez, "Segmentation of Ultrasound Images by Using Quantizer Neural Network" Proceedings of the 15th IEEE Symposium on Computer-Based Medical Systems, 2002. (CBMS 2002). 4-7 June 2002 pp. :257 - 261.

[54] T. Mclnemey and D. Terzopoulos, "Deformable Models in Medical Image Analysis: B survey,” Medical Image Ana/ysis,Vol. 1, pp. 91-108, 1996.

[55] T. McInerney, M.R.A Sharif, "Sketch Initialized Snakes for Rapid, Accurate, and Repeatable Interactive Medical Image Segmentation" 3rd IEEE International Symposium on Biomedical Imaging, 6-9 April 2006 pp. 398 - 401.

[56] P. He; J Zheng, "Segmentation of Tibia Bone in Ultrasound Images Using Active Shape Models", Engineering in Medicine and Biology Society, 2001. Proceedings of the 23rd Annual International Conference of the IEEE Vol. 3, 25-28 Oct. 2001 pp. $2712-2715$.

[57] S. Ourselin, R. Li, "Extension of Deformable Models: Hybrid Approaches for Analysis of Medical Images" ,Engineering in Medicine and Biology Society, 2005. IEEE-EMBS 2005. 27th Annual International Conference of the 01-04 Sept. 2005 pp. $7182-7185$.

[58] K.M. Lam, and H. Yan "Locating and Extracting the Eye in Human Face Images " Pattern Recognition, Vol. 29, Issue 5, May 1996, pp. 771-779.

[59] Y. Wu; H. Liu; H. Zha; "A New Method of Detecting Human Eyelids Based on Deformable Templates" , Conference on Systems, Man and Cybernetics, 2004 IEEE International, Vol. 1, 10-13 Oct. 2004 pp. $604-609$.

[60] S. Sclaroff, L. Liu , "Deformable Shape Detection and Description via Model-based Region Grouping", IEEE Transactions on Pattern Analysis and Machine Intelligence, Vol. 23, Issue 5, May 2001 pp. $475-489$.

[61] M.H. Yang; D.J. Kriegman, N. Ahuja "Detecting Faces in Images: a Survey" , IEEE Transactions on Pattern Analysis and Machine Intelligence, Vol. 24, Issue 1, Jan. 2002 pp. $34-58$.

[62] C. R. Giardina, E. Dougherty,"Morphological Methods in Image and Signal Processing" Printice Hall 1988.

[63] P. Maragos and R. W. Schafer, "Morphological Systems for Multidimensional Signal Processing", Proc. IEEE, Vol. 78, pp. 690-710, 1990.

[64] T. Chen, Q.H. Wu, R. Rahmani-Torkaman, J. Hughes "A Pseudo Top-hat Mathematical Morphological Approach to Edge Detection in Dark Regions", Pattern Recognition, Vol. 35, n. 1, pp. 199-210. , January 2002.

[65] C.K. Lee, S.P. Wong "A Mathematical Morphological Approach for Segmenting Heavily Noise-Corrupted Images”,Pattern Recognition, Vol. 29, n. 8, pp. 1347-1358, August 1996 
[66] R.S. Lin , C.H. Chu , Y.C. Hsueh, A modified Morphological Corner Detector, Pattern Recognition Letters, Vol. 19 n.3-4, pp.279-286, March 1998

[67] R. Laganiere," A Morphological Operator For Corner Detection, Pattern Recognition, Vol. 31 n.11, pp. 1643-1652. , 1998.

[68] G. Louverdis, M.I. Vardavoulia, Andreadis, "A New Approach to Morphological Color Image Processing" Pattern Recognition, Vol. 35, Number 8 , pp. 1733-1741(9) ,August 2002.

[69] K. Held, E. R. Kops, B. J. Krause, W. M. Wells, and R. Kikinis, "Markov Random Field Segmentation of Brain MR Images", IEEE Trans. Medical Imaging, Vol. 16, pp. 878-886, 1997.

[70] J.S. Lin, K.S. Cheng and C.W. Mao, "L fuzzy Hopfield Neural Network for Medical Image Segmentation", IEEE Transactions on Nuclear Science, Vol. 43 , no. 4 , pp. 23892398, 1996.

[71] S. Shiffman, G. D. Rubin, and S. Naple "Medical Image Segmentaion Using Analysis of Isolable - Contour Maps", IEEE Trans. Medical Imaging, Vol. 19, Issue:11, Nov. 2000 pp. 1064-1074.

[72] V. Grau, A.U.J. Mewes, M. Alcaniz, R. Kikinis, S.K.Warfield, ”Improved Watershed Transform for Medical Image Segmentation Using Prior Information" IEEE Trans. Medical Imaging, Vol. 23, Issue:4, April. 2004 pp. 447-458.

[73] Y. J. Zhang, "A Survey on Evaluation Methods for Image Segmentation", Pattern Recognition, Vol. 29 , no. 8, pp. 1335-1346, 1996.

[74] R. M. Haralick and L. G. Shapiro, "Computer and Robot Vision”. Addison-Wesley, New York (1992).

[75] E. Abdou and W. K. Pratt, "Quantitative Design and Evaluation of Enhancement/Thresholding Edge Detectors", Proc. IEEE 67, pp. 753-763 (1979).

[76] C. Garbay, "Image Structure Representation and Processing: a Discussion of Some Segmentation Methods in Cytology", IEEE Trans. PAMI-8, pp. 140-146 (1986).

[77] C.E. Liedtke, T. Gahm, F. Kappei, B. Aeikens, "Segmentation of Microscopic Cell Scenes", AQCH 9, pp. 197-211 (1987).

[78] P. K. Sahoo, S. Soltani, A. K, C. Wong, Y. C. Chen, "A Survey of Thresholding Techniques', CVGIP 41, pp. 233 - 260 (1988).

[79] N. R. Pal and S. K. Pal, “Entropic Thresholding', Sign. Process. 16, pp. 97-108 (1989).

[80] N. Otsu, "A Threshold Selection Method from Gray-Level Histogram", IEEE Trans. SMC9, pp. 62-66 (1979).

[81] W. K. Pratt, "Digital Image Processing”. John Wiley and Sons, New York (1978). 
[82] W. A. Yasnoff and J. W. Bacus, "Scene Segmentation Algorithm Development Using Error Measures", AOCH 6, pp. 45-58 (1984).

[83] M. D. Levine and A. Nazif, “An Experimental Rule Based System for Testing Low Level Segmentation Strategies, in Multi- Computers and Image Processing: Algorithms and Programs", K. Preston and L. Uhr, eds., pp. 149-160. Academic Press, New York (1982).

[84] D. E. M. Nassar, F. U. Chaudhry, and H. H. Ammar, "On Performance Evaluation of Image Segmentation Algorithms: Success is Not All or None", Proc. the 1st International Computer Engineering Conference (ICENCO’2004), pp. 354-359, Egypt, Dec. 2004.

[85] M. Abdel-Mottaleb and J. Zhou, "A System for Ear Biometrics from Face Profile Images.

[86] H. Chen, and B. Bhanu, "Human Ear Detection from Side Face Range Images" Pattern Recognition, 2004. ICPR 2004. Proceedings of the 17th International Conference onVol. 3, 23-26 Aug. 2004 pp. $574-577$.

[87] H. Chen, and B. Bhanu, "Shape Model-Based 3D Ear Detection from Side Face Range Images" Computer Vision and Pattern Recognition, 2005 IEEE Computer Society Conference onVol. 3, 20-26 June 2005 pp. $122-122$.

[88] M.H. Yang, D. J. Kriegman, and N. Ahuja, "Detecting Faces in Images: A Survey" IEEE Transaction on Pattern Analysis and Machine Intelligence, Vol. 24, NO. 1, JANUARY 2002.

[89] R. C. Gonzales and R. E. Woods, Digital Image Processing, Second Edition, Prentice Hall, 2002.

[90] P. Maragos and R. W. Schafer, "Morphological Systems for Multidimensional Signal Processing”, Proc. IEEE, Vol. 78, pp. 690-710, 1990.

[91] Mary L. Comer and Edward J. Delp. "Morphological Operators for ColorImage Processing". Journal of Electronic Image, Vol.8, number(3), pp. 279-289, July 1999.

[92] J.J.G. Leandro, RM. Cesar, H.F. Jelinek, Blood Vessels Segmentation in Retina: Preliminary Assessment of the Mathematical Morphology and of the Wavelet Transform Techniques, Computer Graphics and Image Processing, 2001 Proceedings of XIV Brazilian Symposium on 15-18 Oct. 2001 pp. $84-90$.

[93] F. Zana, J. C. Klein, Robust segmentation of Vessels from Retinal Angiography, Digital Signal Processing Proceedings, 1997. DSP 97., 1997 13th International Conference on Volume 2, 2-4 July 1997 pp. 1087 - 1090 Vol.2.

[94] G.K. Matsopoulos, S. Marshall, Medical Applications of Mathematical Morphology, Morphological and Nonlinear Image Processing Techniques, IEE Colloquium on 10 Jun 1993 pp. $8 / 1-8 / 4$.

[95] J. Samarabandu, R. Acharya, E. Hausmann, and K. Allen, Analysis of Bone X-rays Using Morphological Fractals, IEEE Transactions on Medical Imaging, Vol. 12 (1993), number 3 pp. 466-470. 
[96] A.S.A. Souza, E.E.S. Ruiz, Fast and Accurate Detection of Extraocular Muscle Borders Using Mathematical Morphology, Engineering in Medicine and Biology Society, 2000. Proceedings of the 22nd Annual International Conference of the IEEE, Vol. 3, 23-28 July 2000 pp. 1779 - 1782 Vol.3.

[97] B. Dogdas, DW. Shattuck, RM. Leahy, "Segmentation of the Skull in 3D Human MR Images Using Mathematical Morphology, Proc SPIE Medical Imaging Conf, 2002; 4684 pp.1553-1562. (2002).

[98] K.I. Siddiqui, A.O. Hero, M.M. Siddiqui, Mathematical Morphology Applied to Spot Segmentation and Quantification of Gene Microarray Images, Signals, Systems and Computers, 2002. Conference Record of the Thirty-Sixth Asilomar Conference on Vol. 1, 3-6 Nov. 2002 pp. 926 - 930 Vol. 1.

[99] I.T. Jolliffe, Principal Component Analysis, Second Edition, Springer, 2002.

[100] P. J. Phillips, H. Moon, P. Rauss, and S. A. Rizvi. The FERET Evaluation Methodology for Face-Recognition Algorithms. Pattern Analysis and Machine Intelligence, IEEE Transactions on Vol. 22, Issue 10, Oct. 2000 pp. 1090 - 1104.

[101] S. Buchala, N. Davey, R.J. Frank, TM. Gale, Dimensionality Reduction of Face Imagesfor Gender Classification Intelligent Systems, 2004. Proceedings. 2004 2nd International IEEE Conference Vol. 1, 22-24 June 2004 pp. 88 - 93 Vol.1.

[102] H. Cevikalp, M. Neamtu, M. Wilkes, A. Barkana, Discriminative Common Vectors for Face Recognition, Pattern Analysis and Machine Intelligence, IEEE Transactions on Vol. 27, Issue 1, Jan 2005 pp. 4 - 13.

[103] C. Liu; Gabor-based Kernel PCA with Fractional Power Polynomial Models for Face Recognition Pattern Analysis and Machine Intelligence, IEEE Transactions on Vol. 26, Issue 5, May 2004 pp. $572-581$.

[104] K.-K. Sung, T. Poggio, Example-based Learning for View-based Human Face Detection, Pattern Analysis and Machine Intelligence, IEEE Transactions on Vol. 20, Issue 1, Jan. 1998 pp. $39-51$.

[105] H. Moon, P.J. Phillips, Computational and Performance Aspects of PCA-based Face Recognition Algorithms, Perception, Vol. 30, 2001, pp. 303-321.

[106] M. Turk, A. Pentland,"Face Recognition Using Eigenfaces", Proceedings of the 1991 IEEE Computer Society Conference on Computer Vision and Pattern Recognition, 1991, pp. 586591.

[107] M. Turk and A. Pentland, "Eigenfaces for recognition," Journal of Cognitive Neuroscience, Vol. 3, No. 1, pp. 71-86, Winter 1991.

[108] A. Pentland, B. Moghaddam, T. Starner, View-Based and Modular Eigenspaces for Face Recognition, Proceedings of the IEEE Conference on Computer Vision and Pattern Recognition, 21-23 June 1994, Seattle, Washington, USA, pp. 84-91.

[109] A.N. Rajagopalan, R. Chellappa, N.T. Koterba, Background Learning for Robust Face 
Recognition with PCA in the Presence of Clutter Image Processing, IEEE Transactions on Vol. 14, Issue 6, June 2005 pp. $832-843$.

[110] D. Nassar," Automated Dental Identification: A Macro Decision - Making Approach" ,PHD thesis, 2005.

[111] E. Haj Said, D. Nassar, G. Fahmy, and H. Ammar , “ Teeth Segmentation in Digitized Dental X-Rays Films using Mathematical" IEEE Transactions on Information Forensics and Security, Vol. 1, No. 2, June 2006.

[112] The profile visible part of Database collected for the purpose of face and ear recognition at the Unviversity of Notredame.

[113] CITeR face database containig 50 subjects, Collected at West Virginia University for purpose of studying the performance of face recognition.

[114] Automated 3-D Ear Identification project database containing 318 subjects, Collected at West Virginia University for of studying ear Identification.

[115] CJIS Division - "Washington State Patrol Missing and Unidentified Person Unit", Digitized Dental Images (Database), May 2000.

[116] CJIS Division - ADIS project, Digitized Radiographic Images (Database), August 2002.

[117] R. Duda, P. Hart, and D. Stork, " Pattern Classification " Second Edition, Wiley, John \& Sons, Incorporated, 2001.

[118] B. Moghaddam, A. Pentland, "Probabilistic Visual Learning For Object Representation" IEEE Transactions on Pattern Analysis and Machine Intelligence, Vol. 19, No. 7, July 1997, pp. 696-710. 